\title{
Pacific
}

Journal of

Mathematics

\section{GENERALIZATIONS OF AGOL'S INEQUALITY AND NONEXISTENCE OF TIGHT LAMINATIONS}

\author{
THILO KUESSNER
}




\title{
GENERALIZATIONS OF AGOL'S INEQUALITY AND NONEXISTENCE OF TIGHT LAMINATIONS
}

\author{
THILO KUESSNER
}

\begin{abstract}
We give a general lower bound for the normal Gromov norm of genuine laminations in terms of the topology of the complementary regions.

In the special case of 3-manifolds, this yields a generalization of Agol's inequality from incompressible surfaces to tight laminations. In particular, the inequality excludes the existence of tight laminations with nonempty guts on 3-manifolds of small simplicial volume.
\end{abstract}

\section{Results}

Agol's inequality [1999, Theorem 2.1] is the following (see Section 7 for notation):

Let $M$ be a hyperbolic 3-manifold containing an incompressible, properly embedded surface $F$. Then

$$
\operatorname{Vol}(M) \geq-2 V_{3} \chi(\operatorname{Guts}(\overline{M-F})),
$$

where $V_{3}$ is the volume of a regular ideal tetrahedron in hyperbolic 3-space.

In [Agol et al. 2007], this inequality was improved to

$$
\operatorname{Vol}(M) \geq \operatorname{Vol}(\operatorname{Guts}(\overline{M-F})) \geq-V_{\text {oct }} \chi(\operatorname{Guts}(\overline{M-F})),
$$

where $V_{\text {oct }}$ is the volume of a regular ideal octahedron in hyperbolic 3-space.

In this paper we will, building on ideas from [Agol 1999], prove a general inequality for the (transversal) Gromov norm $\|M\|_{\mathscr{F}}$ and the normal Gromov norm $\|M\|_{\mathscr{F}}^{\text {norm }}$ of laminations.

To state the result in its general form we need two definitions.

Definition (pared acylindrical). Let $Q$ be a manifold with a given decomposition

$$
\partial Q=\partial_{0} Q \cup \partial_{1} Q .
$$

The pair $\left(Q, \partial_{1} Q\right)$ is called a pared acylindrical manifold if any continuous map of pairs $f:\left(\mathbb{S}^{1} \times[0,1], \mathbb{S}^{1} \times\{0,1\}\right) \rightarrow\left(Q, \partial_{1} Q\right)$ that is $\pi_{1}$-injective as a map of pairs

MSC2000: primary 57R30; secondary 53C23, 57M27, 57N10, 57M50.

Keywords: lamination, hyperbolic, 3-manifold, volume, Gromov norm, tight, Weeks manifold, simplicial, simplex, straightening. 
is necessarily homotopic, as a map of pairs $\left(\mathbb{S}^{1} \times[0,1], \mathbb{S}^{1} \times\{0,1\}\right) \rightarrow\left(Q, \partial_{1} Q\right)$, into $\partial Q$.

Definition (essential decomposition). Let $(N, \partial N)$ be a pair of topological spaces such that $N=Q \cup R$ for two subspaces $Q, R$. Let

$\partial_{0} Q=Q \cap R, \partial_{1} Q=Q \cap \partial N, \partial_{1} R=R \cap \partial N, \partial Q=\partial_{0} Q \cup \partial_{1} Q, \partial R=\partial_{0} Q \cup \partial_{1} R$.

We say that the decomposition $N=Q \cup R$ is an essential decomposition of $(N, \partial N)$ if the inclusions

$$
\partial_{1} Q \rightarrow Q \rightarrow N, \partial_{1} R \rightarrow R \rightarrow N, \partial N \rightarrow N, \partial_{0} Q \rightarrow Q, \partial_{0} Q \rightarrow R
$$

are each $\pi_{1}$-injective (for each path component).

Theorem 1.1. Let $M$ be a compact, orientable, connected $n$-manifold and $\mathscr{F} a$ lamination (of codimension one) of $M$. Assume that $N:=\overline{M-\mathscr{F}}$ has a decomposition $N=Q \cup R$ into orientable n-manifolds (with boundary) $Q, R$ such that the following assumptions are satisfied for $\partial_{0} Q=Q \cap R, \partial_{1} Q=Q \cap \partial N, \partial_{1} R=R \cap \partial N$ :

(i) Each path component of $\partial_{0} Q$ has amenable fundamental group.

(ii) $\left(Q, \partial_{1} Q\right)$ is pared acylindrical and $\partial_{1} Q$ is acylindrical.

(iii) $Q, \partial N, \partial_{1} Q, \partial_{1} R, \partial_{0} Q$ are aspherical.

(iv) The decomposition $N=Q \cup R$ is an essential decomposition of $(N, \partial N)$.

Then

$$
\|M, \partial M\|_{\mathscr{F}}^{\text {norm }} \geq \frac{1}{n+1}\|\partial Q\| .
$$

In the case of 3-manifolds $M$ carrying an essential lamination $\mathscr{F}$, considering $Q=\operatorname{Guts}(\overline{M-\mathscr{F}})$ yields a special case:

Theorem 1.2. Let $M$ be a compact 3-manifold with (possibly empty) boundary consisting of incompressible tori, and let $\mathscr{F}$ be an essential lamination of $M$. Then

$$
\|M, \partial M\|_{\mathscr{F}}^{\text {norm }} \geq-\chi(\operatorname{Guts}(\overline{M-\mathscr{F}})) .
$$

More generally, if $P$ is a polyhedron with $f$ faces, then

$$
\|M, \partial M\|_{\mathscr{F}, P}^{\text {norm }} \geq-\frac{2}{f-2} \chi(\operatorname{Guts}(\overline{M-\mathscr{F}})) .
$$

The following corollary applies, for example, to all hyperbolic manifolds $M$ obtained by Dehn-filling the complement of the figure-eight knot in $\mathbb{S}^{3}$. (It is known that each of these $M$ contains tight laminations. By the following corollary, all these tight laminations have empty guts.) 
Corollary 1.3. If $M$ is a finite-volume hyperbolic 3-manifold with $\operatorname{Vol}(M)<2 V_{3}=$ $2.02 \ldots$, then $M$ carries no essential lamination $\mathscr{F}_{\mathcal{F}}$ with $\|M\|_{\mathscr{F}, P}^{\text {norm }}=\|M\|_{P}$ for all polyhedra $P$, and nonempty guts. In particular, there is no tight essential lamination with nonempty guts.

Calegari and Dunfield [2003] observed that their own results about tight laminations with empty guts would imply the following corollary, in the presence of a generalization of Agol's inequality to the case of tight laminations.

Corollary 1.4 [Calegari and Dunfield 2003, Conjecture 9.7]. The Weeks manifold (the closed hyperbolic manifold of smallest volume) admits no tight lamination $\mathscr{F}_{\text {F }}$

Taking into account the main result of [Li 2006], this can be strengthened:

Corollary 1.5. The Weeks manifold admits no transversely orientable essential lamination.

We also have an application of Theorem 1.1 to higher-dimensional manifolds.

Corollary 1.6. Let $M$ be a compact Riemannian n-manifold of negative sectional curvature and finite volume. Let $F \subset M$ be a geodesic $(n-1)$-dimensional hypersurface of finite volume. Then $\|F\| \leq \frac{1}{2}(n+1)\|M\|$.

The basic idea of Theorem 1.1, say for simplicity in the special situation of Corollary 1.6, is the following: a simplex which contributes to a normalized fundamental cycle of $M$ should intersect $\partial Q=2 F$ in at most $n+1$ codimension-one simplices. This is of course not true in general: simplices can wrap around $M$ many times and intersect $F$ arbitrarily often, and even a homotopy rel vertices will not change this. As an obvious example, look at the following situation: Let $\gamma$ be a closed geodesic transverse to $F$, and for some large $N$ let $\sigma$ be a straight simplex contained in a small neighborhood of $\gamma^{N}$. Then $\sigma$ and $F$ intersect $N$ times and, since $\sigma$ is already straight, this number of intersections can of course not be reduced by straightening. This shows that some more involved straightening must take place, and that the acylindricity of $F$ is an essential condition. The way to use acylindricity will be to find a normalization such that many subsets of simplices are mapped to cylinders, which degenerate and thus can be removed without changing the homology class.

We remark that many technical points, including the use of multicomplexes, can be omitted if (in the setting of Theorem 1.2) one does not consider incompressible surfaces or essential laminations, but just geodesic surfaces in hyperbolic manifolds. In this case, all essential parts of the proof of Theorem 1.1 enter without the notational complications caused by the use of multicomplexes. Therefore we have given a fairly detailed outline of the proof for this special case in the beginning of Section 6. This should help to motivate the general proof in the second half of that section (156). (We mention that Theorem 1.1 is not true without assuming 
amenability of $\pi_{1} \partial_{0} Q$. This indicates that the proof of multicomplexes in the proof of Theorem 1.1 seems unavoidable.)

\section{Preliminaries}

2A. Laminations. Let $M$ be an $n$-manifold, possibly with boundary. In this paper all manifolds will be smooth and orientable. (Hence they are triangulable by Whitehead's theorem and possess a locally finite fundamental class.) A (codimension 1) lamination $\mathscr{F}$ of $M$ is a foliation of a closed subset $\mathscr{F}$ of $M$, i.e., a decomposition of a closed subset $\mathscr{F} \subset M$ into immersed codimension 1 submanifolds (leaves) so that $M$ is covered by charts $\phi_{j}: \mathbb{R}^{n-1} \times \mathbb{R} \rightarrow M$, the intersection of any leaf with the image of any chart $\phi_{j}$ being a union of plaques of the form $\phi_{j}\left(\mathbb{R}^{n-1} \times\{*\}\right)$. (We will denote by $\mathscr{F}$ both the lamination and the laminated subset of $M$, i.e., the union of leaves.) If $M$ has boundary, we will always assume without further mentioning that $\mathscr{F}$ is either transverse to $\partial M$ (that is, every leaf is transverse to $\mathscr{F}$ ) or tangential to $\partial M$ (that is, $\partial M$ is a leaf of $\mathscr{F}$ ). If neither of these two conditions were true, then the transverse and normal Gromov norm would be infinite, therefore all lower bounds will be trivially true.

To construct the leaf space $T$ of $\mathscr{F}$, one considers the pull-back lamination $\widetilde{\mathscr{F}}$ on the universal covering $\tilde{M}$. The space of leaves $T$ is defined as the quotient of $\tilde{M}$ under the following equivalence relation $\sim$. Two points $x, y \in \widetilde{M}$ are equivalent if either they belong to the same leaf of $\widetilde{\mathscr{F}}$, or they belong to the same connected component of the metric completion $\widetilde{\widetilde{M}-\widetilde{\mathscr{F}}}$ (for the path metric inherited by $\widetilde{M}-\widetilde{\mathscr{F}}$ from an arbitrary Riemannian metric on $\widetilde{M}$ ).

2B. Laminations of 3-manifolds. A lamination $\mathscr{F}$ of a 3-manifold $M$ is called essential if no leaf is a sphere or a torus bounding a solid torus, $\overline{M-\mathscr{F}}$ is irreducible, and $\partial(\overline{M-\mathscr{F}})$ is incompressible and end-incompressible in $\overline{M-\mathscr{F}}$, where again the metric completion $\overline{M-\mathscr{F}}$ of $M-\mathscr{F}$ is taken with respect to the path metric inherited from any Riemannian metric on $M$; see [Gabai and Oertel 1989, Chapter 1]. (Note that $\overline{M-\mathscr{F}}$ is immersed in $M$, the leaves of $\mathscr{F}$ in the image of the immersion are called boundary leaves.)

Examples of essential laminations are taut foliations or compact, incompressible, boundary-incompressible surfaces in compact 3-manifolds. (We always consider laminations without isolated leaves. If a lamination has isolated leaves, then it can be converted into a lamination without isolated leaves by replacing each twosided isolated leaf $S_{i}$ with the trivially foliated product $S_{i} \times[0,1]$, resp. each onesided isolated leaf with the canonically foliated normal $I$-bundle, without changing the topological type of $M$.)

If $\mathscr{F}$ is an essential lamination, then the leaf space $T$ is an order tree, with segments corresponding to directed, transverse, efficient arcs. (An order tree $T$ is 
a set $T$ with a collection of linearly ordered subsets, called segments, such that the axioms of [Gabai and Oertel 1989, Definition 6.9], are satisfied.) Moreover, $T$ is an $\mathbb{R}$-order tree, that is, it is a countable union of segments and each segment is order isomorphic to a closed interval in $\mathbb{R}$. $T$ can be topologized by the order topology on segments (and declaring that a set is closed if the intersection with each segment is closed). For this topology, $\pi_{0} T$ and $\pi_{1} T$ are trivial (see, for example, [Roberts et al. 2003], Chapter 5, and its references).

The order tree $T$ comes with a fixed-point free action of $\pi_{1} M$. Fenley [2007] has exhibited hyperbolic 3-manifolds whose fundamental groups do not admit any fixed-point free action on $\mathbb{R}$-order trees. Thus there are hyperbolic 3-manifolds not carrying any essential lamination.

If $M$ is hyperbolic and $\mathscr{F}$ an essential lamination, then $\overline{M-\mathscr{F}}$ has a characteristic submanifold which is the maximal submanifold that can be decomposed into $I$ bundles and solid tori, respecting boundary patterns (see [Jaco and Shalen 1979], [Johannson 1979] for precise definitions). The complement of this characteristic submanifold is denoted by Guts(F) $(\mathscr{F})$ It admits a hyperbolic metric with geodesic boundary and cusps. (Be aware that some authors, like [Calegari and Dunfield 2003], include the solid tori into the guts.) If $\mathscr{F}=F$ is a properly embedded, incompressible, boundary-incompressible surface, then Agol's inequality states that $\operatorname{Vol}(M) \geq-2 V_{3} \chi(\operatorname{Guts}(F))$. This implies, for example, that a hyperbolic manifold of volume $<2 V_{3}$ can not contain any geodesic surface of finite area. Agol, Storm, and Thurston [Agol et al. 2007], using estimates coming from Perelman's work on the Ricci flow, have improved this inequality to

$$
\operatorname{Vol}(M) \geq \operatorname{Vol}(\operatorname{Guts}(F)) \geq-V_{\text {oct }} \chi(\operatorname{Guts}(F)) .
$$

Assume that $\mathscr{F}$ is a codimension one lamination of an $n$-manifold $M$ such that its leaf space $T$ is an $\mathbb{R}$-order tree. (For example this is the case if $n=3$ and $\mathscr{F}$ is essential.) An essential lamination is called tight if $T$ is Hausdorff. It is called unbranched if $T$ is homeomorphic to $\mathbb{R}$. It is said to have two-sided branching [Calegari 2000, Definition 2.5.2] if there are leaves $\lambda, \lambda_{1}, \lambda_{2}, \mu, \mu_{1}, \mu_{2}$ such that the corresponding points in the $T$ satisfy $\lambda<\lambda_{1}, \lambda<\lambda_{2}, \mu>\mu_{1}, \mu>\mu_{2}$, but $\lambda_{1}, \lambda_{2}$ are incomparable and $\mu_{1}, \mu_{2}$ are incomparable. It is said to have one-sided branching if it is neither unbranched nor has two-sided branching.

If $M$ is a hyperbolic 3-manifold and carries a tight lamination with empty guts, we know from [2003, Theorem 3.2] that $\pi_{1} M$ acts effectively on the circle, i.e., there is an injective homomorphism $\pi_{1} M \rightarrow \operatorname{Homeo}\left(\mathbb{S}^{1}\right)$. This implies that the Weeks manifold cannot carry a tight lamination with empty guts [Calegari and Dunfield 2003, Corollary 9.4]. The aim of this paper is to find obstructions to the existence of laminations with nonempty guts. 
2C. Simplicial volume and refinements. Let $M$ be a compact, orientable, connected $n$-manifold, possibly with boundary. Its top integer (singular) homology group $H_{n}(M, \partial M ; \mathbb{Z})$ is cyclic. The image of a generator under the change-ofcoefficients homomorphism $H_{n}(M, \partial M ; \mathbb{Z}) \rightarrow H_{n}(M, \partial M ; \mathbb{R})$ is called a fundamental class and is denoted $[M, \partial M]$. If $M$ is not connected, we define $[M, \partial M]$ to be the formal sum of the fundamental classes of its connected components.

The simplicial volume $\|M, \partial M\|$ is defined as

$$
\|M, \partial M\|=\inf \left\{\sum_{i=1}^{r}\left|a_{i}\right|\right\},
$$

where the infimum is taken over all singular chains $\sum_{i=1}^{r} a_{i} \sigma_{i}$ (with real coefficients) representing the fundamental class in $H_{n}(M, \partial M ; \mathbb{R})$.

If $M-\partial M$ carries a complete hyperbolic metric of finite volume $\operatorname{Vol}(M)$, then

$$
\|M, \partial M\|=\frac{1}{V_{n}} \operatorname{Vol}(M),
$$

with $V_{n}=\sup \left\{\operatorname{Vol}(\Delta): \Delta \subset \mathbb{H}^{n}\right.$ geodesic simplex $\}$; see [Gromov 1982; Thurston 1980; Benedetti and Petronio 1992; Francaviglia 2004].

More generally, let $P$ be any polyhedron. Then the invariant $\|M, \partial M\|_{P}$ is defined in [Agol 1999] as follows: denoting by $C_{*}(M, \partial M ; P ; \mathbb{R})$ the complex of $P$-chains with real coefficients and by $H_{*}(M, \partial M ; P ; \mathbb{R})$ its homology, there is a canonical chain homomorphism $\psi: C_{*}(M, \partial M ; P ; \mathbb{R}) \rightarrow C_{*}(M, \partial M ; \mathbb{R})$, given by certain triangulations of $P$ which are to be chosen so that all possible cancellations of boundary faces are preserved. Then $\|M, \partial M\|_{P}$ is defined as the infimum of $\sum_{i=1}^{r}\left|a_{i}\right|$ over all $P$-chains $\sum_{i=1}^{r} a_{i} P_{i}$ such that $\psi\left(\sum_{i=1}^{r} a_{i} P_{i}\right)$ represents the fundamental class $[M, \partial M]$. Set $V_{P}:=\sup \{\operatorname{Vol}(\Delta)\}$, where the supremum is taken over all straight $P$-polyhedra $\Delta \subset \mathbb{Q}^{3}$.

Proposition 2.1 [Agol 1999, Lemma 4.1]. If $M-\partial M$ admits a hyperbolic metric of finite volume $\operatorname{Vol}(M)$, then

$$
\|M, \partial M\|_{P}=\frac{1}{V_{P}} \operatorname{Vol}(M) .
$$

(The proof in [Agol 1999] is quite short, and it does not give details for the cusped case. However, the proof in the cusped case can be completed using the arguments in [Francaviglia 2004, Sections 5 and 6].)

Let $M$ be a manifold and $\mathscr{F}$ a codimension-one lamination of $M$. Let $\Delta^{n}$ be the standard simplex in $\mathbb{R}^{n+1}$, and $\sigma: \Delta^{n} \rightarrow M$ some continuous singular simplex. The lamination $\mathscr{F}$ induces an equivalence relation on $\Delta^{n}$, whereby $x \sim y$ if and only if $\sigma(x)$ and $\sigma(y)$ belong to the same connected component of $L \cap \sigma\left(\Delta^{n}\right)$ for some leaf $L$ of $\mathscr{F}$. We say that a singular simplex $\sigma: \Delta^{n} \rightarrow M$ is laminated if the 
equivalence relation $\sim$ is induced by a lamination $\left.\mathscr{F}\right|_{\sigma}$ of $\Delta^{n}$. We call a lamination $\mathscr{F}_{F}$ of $\Delta^{n}$ affine if there is an affine mapping $f: \Delta^{n} \rightarrow \mathbb{R}$ such that $x, y \in \Delta^{n}$ belong to the same leaf if and only if $f(x)=f(y)$. We say that a lamination $\varphi$ of $\Delta^{n}$ is conjugate to an affine lamination if there is a simplicial homeomorphism $H: \Delta^{n} \rightarrow \Delta^{n}$ such that $H^{*} \varphi$ is an affine lamination.

We say that a singular $n$-simplex $\sigma: \Delta^{n} \rightarrow M, n \geq 2$, is transverse to $\mathscr{F}_{F}$ if it is laminated and it is either contained in a leaf, or $\left.\mathscr{F}\right|_{\sigma}$ is conjugate to an affine lamination $\varphi$ of $\Delta^{n}$.

For $n=1$, we say that a singular 1-simplex $\sigma: \Delta^{1} \rightarrow M$ is transverse to $\mathscr{F}$ if it is either contained in a leaf, or for each lamination chart $\phi: U \rightarrow \mathbb{R}^{m-1} \times \mathbb{R}^{1}$ (with m-th coordinate map $\left.\phi_{m}: U \rightarrow \mathbb{R}^{1}\right)$ one has that $\left.\phi_{m} \circ \sigma\right|_{\sigma^{-1}(U)}: \sigma^{-1}(U) \rightarrow \mathbb{R}^{1}$ is locally surjective at all points of $\operatorname{int}\left(\Delta^{1}\right)$, i.e., for all $p \in \operatorname{int}\left(\Delta^{1}\right) \cap \sigma^{-1}(U)$, the image of $\left.\phi_{m} \circ \sigma\right|_{\sigma^{-1}(U)}$ contains a neighborhood of $\phi_{m} \circ \sigma(p)$.

We say that the simplex $\sigma: \Delta^{n} \rightarrow M$ is normal to $\mathscr{F}$ if, for each leaf $F, \sigma^{-1}(F)$ consists of normal disks, i.e., disks meeting each edge of $\Delta^{n}$ at most once. (If $F=\partial M$ is a leaf of $\mathscr{F}$ we also allow that $\sigma^{-1}(F)$ can be a face of $\left.\Delta^{n}\right)$. In particular, any transverse simplex is normal.

In the special case of foliations, $\mathscr{F}$ one has that the transversality of a singular simplex $\sigma$ is implied by (hence equivalent to) the normality of $\sigma$, as can be shown along the lines of [Kuessner 2004, Section 1.3].

More generally, let $P$ be any polyhedron. Then we say that a singular polyhedron $\sigma: P \rightarrow M$ is normal to $\mathscr{F}$ if, for each leaf $F, \sigma^{-1}(F)$ consists of normal disks, i.e., disks meeting each edge of $P$ at most once (or being equal to a face of $P$, if $F$ is a boundary leaf).

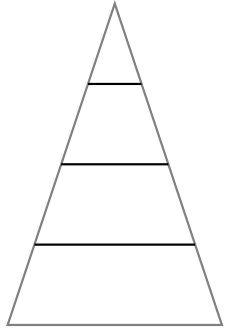

transverse

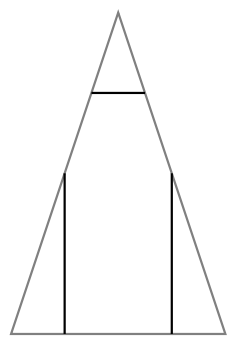

normal, not transverse

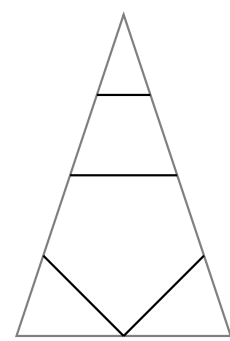

not normal

Definition 2.2. Let $M$ be a compact, oriented, connected $n$-manifold, possibly with boundary, and let $\mathscr{F}_{F}$ be a foliation or lamination on $M$. Let $\Delta^{n}$ be the standard simplex and $P$ any polyhedron. Let $\Sigma$ be the set of singular simplices $\Delta^{n} \rightarrow M$ transverse to $\mathscr{F}$. We define

$\|M, \partial M\|_{\mathscr{F}}:=\inf \left\{\sum_{i=1}^{r}\left|a_{i}\right|: \psi\left(\sum_{i=1}^{r} a_{i} \sigma_{i}\right)\right.$ represents $[M, \partial M]$ for some $\left.\sigma_{i} \in \Sigma\right\}$ 
and

$\|M, \partial M\|_{\mathscr{F}, P}^{\text {norm }}:=\inf \left\{\sum_{i=1}^{r}\left|a_{i}\right|: \psi\left(\sum_{i=1}^{r} a_{i} \sigma_{i}\right)\right.$ represents $[M, \partial M]$ for some $\left.\sigma_{i} \in \Sigma\right\}$. In particular, we define $\|M, \partial M\|_{\mathscr{F}}^{\text {norm }}=\|M, \partial M\|_{\mathscr{F}, \Delta^{n}}^{\text {norm }}$.

All these norms are finite, under the assumption that $\mathscr{F}$ is transverse or tangential to $\partial M$. There are obvious inequalities

$$
\|M, \partial M\| \leq\|M, \partial M\|_{\mathscr{F}}^{\text {norm }} \leq\|M, \partial M\|_{\mathscr{F}} .
$$

In the case of foliations, this last inequalities becomes an equality.

(We remark that all definitions extend in an obvious way to disconnected manifolds by summing over the connected components.)

The next proposition and lemma are straightforward generalizations of [Calegari 2000, Theorem 2.5.9] and of arguments in [Agol 1999].

Proposition 2.3. Let $M$ be a compact, oriented 3-manifold.

(a) If F is an essential lamination which is either unbranched or has one-sided branching such that the induced lamination of $\partial M$ is unbranched, then

$$
\|M, \partial M\|_{\mathscr{F}, P}^{\text {norm }}=\|M, \partial M\|_{P}
$$

for each polyhedron $P$.

(b) If $\mathscr{F}$ is a tight essential lamination, then

$$
\|M, \partial M\|_{\mathscr{F}, P}^{\text {norm }}=\|M, \partial M\|_{P}
$$

for each polyhedron $P$.

Proof. Since $\mathscr{F}$ is an essential lamination, we know from [Gabai and Oertel 1989, Theorem 6.1] that the leaves are $\pi_{1}$-injective, the universal covering $\widetilde{M}$ is homeomorphic to $\mathbb{R}^{3}$ and that the leaves of the pull-back lamination are planes, in particular aspherical. Therefore Proposition 2.3 is a special case of the next result.

Lemma 2.4. Let $M$ be a compact, oriented, aspherical manifold, and F a lamination of codimension one. Assume that the leaves are $\pi_{1}$-injective and aspherical, and that the leaf space $T$ is an $\mathbb{R}$-order tree.

(a) If the leaf space $T$ is either $\mathbb{R}$ or branches in only one direction, so that the induced lamination of $\partial M$ has leaf space $\mathbb{R}$, then $\|M, \partial M\|_{\mathscr{F}, P}^{\text {norm }}=\|M, \partial M\|_{P}$ for each polyhedron $P$.

(b) If the leaf space is a Hausdorff tree, then $\|M, \partial M\|_{\mathscr{F}, P}^{\text {norm }}=\|M, \partial M\|_{P}$ for each polyhedron $P$. 
Proof. To prove the wanted equalities, it suffices in each case to show that any (relative) cycle can be homotoped to a cycle consisting of normal polyhedra. We denote by $\widetilde{\mathscr{F}}$ the pull-back lamination of $\widetilde{M}$ and by $p: \widetilde{M} \rightarrow T=\widetilde{M} / \widetilde{\mathscr{F}}$ the projection to the leaf space.

(a) First we consider the case that $P$ is a simplex [Calegari 2000, Section 4.1] and $\mathscr{F}$ is unbranched. For this case, we can repeat the argument in [Calegari 2000, Lemma 2.2.8]. Namely, given a (relative) cycle $\sum_{i=1}^{r} a_{i} \sigma_{i}$, lift it to a $\pi_{1} M$-equivariant (relative) cycle on $\widetilde{M}$ and then perform an (equivariant) straightening, by induction on the dimension of subsimplices of the lifts $\tilde{\sigma}_{i}$ as follows: for each edge $\tilde{e}$ of any lift $\tilde{\sigma}_{i}$, its projection $p(\tilde{e})$ to the leaf space $T$ is homotopic to a unique straight arc $\operatorname{str}(p(\tilde{e}))$ in $T \simeq \mathbb{R}$. It is easy to see (covering the arc by foliation charts and then extending the lifted arc stepwise) that $\operatorname{str}(p(\tilde{e}))$ can be lifted to an $\operatorname{arc} \operatorname{str}(\tilde{e})$ with the same endpoints as $\tilde{e}$, and that the homotopy between $\operatorname{str}(p(\tilde{e}))$ and $p(\tilde{e})$ can be lifted to a homotopy between $\operatorname{str}(\tilde{e})$ and $\tilde{e} \cdot \operatorname{str}(\tilde{e})$ is transverse to $\mathscr{F}$, because its projection is a straight arc in $T$. These homotopies of edges can be extended to a homotopy of the whole (relative) cycle. Thus we have straightened the 1-skeleton of the given (relative) cycle.

Now let us be given a 2-simplex $\tilde{f}: \Delta^{2} \rightarrow \tilde{M}$ with transverse edges. There is an obvious straightening $\operatorname{str}(p(\tilde{f}))$ of $p(\tilde{f}): \Delta^{2} \rightarrow T$ as follows: if, for $t \in T$, $(p \tilde{f})^{-1}(t)$ has two preimages $x_{1}, x_{2}$ on edges of $\Delta^{2}$ (which are necessarily unique), then $\operatorname{str}(p(\tilde{f}))$ maps the line which connects $x_{1}$ and $x_{2}$ in $\Delta^{2}$ constantly to $t$. It is clear that this defines a continuous map $\operatorname{str}(p(\tilde{f})): \Delta^{2} \rightarrow T$.

Since the leaves $\widetilde{F}$ of $\widetilde{\mathscr{F}}$ are connected $\left(\pi_{0} \widetilde{F}=0\right), \operatorname{str}(p(\tilde{f}))$ can be lifted to a map $\operatorname{str}(\tilde{f}): \Delta^{2} \rightarrow \widetilde{M}$ with $p(\operatorname{str}(\tilde{f}))=\operatorname{str}(p(\tilde{f}))$. The 2 -simplex $\operatorname{str}(\tilde{f})$ is transverse to $\mathscr{F}$, because its projection is a straight simplex in $T$.

There is an obvious homotopy between $p(\tilde{f})$ and $\operatorname{str}(p(\tilde{f}))$. For each $t \in T$, the restriction of the homotopy to $(p \tilde{f})^{-1}(t)$ can be lifted to a homotopy in $\tilde{M}$, because $\pi_{1} \tilde{M}=0$. Since $\pi_{2} \widetilde{M}=0$, these homotopies for various $t \in T$ fit together continuously to give a homotopy between $\tilde{f}$ and $\operatorname{str}(\tilde{f})$.

These homotopies of 2-simplices leave the (already transverse) boundaries pointwise fixed; thus they can be extended to a homotopy of the whole (relative) cycle. Hence we have straightened the 2-skeleton of the given (relative) cycle.

Assume that we have already straightened the $k$-skeleton, for some $k \in \mathbb{N}$. The analogous procedure, using $\pi_{k-1} \widetilde{F}=0$ for all leaves, and $\pi_{k} \widetilde{M}=0, \pi_{k+1} \tilde{M}=0$, allows to straighten the $(k+1)$-skeleton of the (relative) cycle. This finishes the proof in the case that $\mathscr{F}$ is unbranched.

The generalization to the case that $\mathscr{F}$ has one-sided branching and the induced lamination of $\partial M$ is unbranched works as in [Calegari 2000, Theorem 2.6.6].

We remark that in the case that $P$ is a simplex we get not only a normal cycle, but even a transverse cycle. 
Now we consider the case of arbitrary polyhedra $P$. Let $\sum_{i=1}^{r} a_{i} \sigma_{i}$ be a $P$ cycle. It can be subtriangulated to a simplicial cycle $\sum_{i=1}^{r} a_{i} \sum_{j=1}^{s} \tau_{i, j}$. Again the argument in [Calegari 2000, Lemma 2.2.8], and the corresponding argument for manifolds with boundary, shows that this simplicial cycle can be homotoped such that each $\tau_{i, j}$ is transverse (and such that boundary cancellations are preserved). But transversality of each $\tau_{i, j}$ implies by definition that $\sigma_{i}=\sum_{j=1}^{s} \tau_{i, j}$ is normal (though in general not transverse) to $\mathscr{F}$.

(b) By assumption $\widetilde{M} / \widetilde{\mathscr{F}}$ is a Hausdorff tree. Its branching points are the projections of complementary regions: Indeed, if $F$ is a leaf of $\mathscr{F}$, then $\widetilde{F}$ is a submanifold of the contractible manifold $\widetilde{M}$. By asphericity and $\pi_{1}$-injectivity of $F, \widetilde{F}$ must be contractible. By Alexander duality it follows that $\widetilde{M}-\widetilde{F}$ has two connected components. Therefore the complement of the point $p(\widetilde{F})$ in the leaf space has (at most) two connected components, so $p(\widetilde{F})$ cannot be a branch point.

Again, to define a straightening of $P$-chains it suffices to define a canonical straightening of singular polyhedra $P$ such that straightenings of common boundary faces will agree. Let $\tilde{v}_{0}, \ldots, \tilde{v}_{n}$ be the vertices of the image of $P$. For each pair $\left\{\tilde{v}_{i}, \tilde{v}_{j}\right\}$ there exists at most one edge $\tilde{e}_{i j}$ with vertices $\tilde{v}_{i}, \tilde{v}_{j}$ in the image of $P$. Since the leaf space is a tree, we have a unique straight $\operatorname{arc} \operatorname{str}\left(p\left(\tilde{e}_{i j}\right)\right)$ connecting the points $p\left(\tilde{v}_{i}\right)$ and $p\left(\tilde{v}_{j}\right)$ in the leaf space. As in (a), one can lift this straight $\operatorname{arc} \operatorname{str}\left(p\left(\tilde{e}_{i j}\right)\right)$ to an $\operatorname{arc} \operatorname{str}\left(\tilde{e}_{i j}\right)$ in $\tilde{M}$, connecting $\tilde{v}_{i}$ and $\tilde{v}_{j}$, which is transverse to $\mathscr{F}$. We define this $\operatorname{arc} \operatorname{str}\left(\tilde{e}_{i j}\right)$ to be the straightening of $\tilde{e}_{i j}$. As in (a), we have homotopies of 1-simplices, which extend to a homotopy of the whole (relative) cycle. Thus we have straightened the 1-skeleton.

Now let us be given the 3 vertices $\tilde{v}_{0}, \tilde{v}_{1}, \tilde{v}_{2}$ of a 2 -simplex $\tilde{f}$ with straight edges. If the projections $p\left(\tilde{v}_{0}\right), p\left(\tilde{v}_{1}\right), p\left(\tilde{v}_{2}\right)$ belong to a subtree isomorphic to a connected subset of $\mathbb{R}$, then we can straighten $\tilde{f}$ as in (a). If not, the projection of the 1-skeleton of this simplex has exactly one branch point, which corresponds to a complementary region. (The projection may of course meet many branch points of the tree, but the image of the projection, considered as a subtree, can have at most one branch point. In general, a subtree with $n$ vertices can have at most $n-2$ branch points.) The preimage of the complement of this complementary region consists of three connected subsets of the 2-simplex (the "corners" around the vertices). We can straighten each of these subsets and do not need to care about the complementary region corresponding to the branch point. Thus we have straightened the 2-skeleton.

Assume that we have already straightened the $k$-skeleton, for some $k \in \mathbb{N}$. Given the $k+2$ vertices $\tilde{v}_{0}, \tilde{v}_{1}, \ldots, \tilde{v}_{k+1}$ of a $(k+1)$-simplex with straight faces, we have (at most $k$ ) branch points in the projection of the simplex, which correspond to complementary regions. Again we can straighten the parts of the simplex which 
do not belong to these complementary regions as in (a), since they are projected to linearly ordered subsets of the tree. Thus we have straightened the $(k+1)$-skeleton.

Since, by the recursive construction, we have defined straightenings of simplices with common faces by first defining (the same) straightenings of their common faces, the straightening of a (relative) cycle will be again a (relative) cycle, in the same (relative) homology class.

Remark. For $\|M\|_{\mathscr{F}}$ instead of $\|M\|_{\mathscr{F}}^{\text {norm }}$, equality (b) is in general wrong, and equality (a) is unknown (but presumably wrong).

If $\mathscr{F}$ is essential but not tight, one may still try to homotope cycles to be transverse, by possibly changing the lamination. In the special case that the cycle is coming from a triangulation, this has been done by Brittenham [1995] and Gabai [1999]. It is not obvious how to generalize their arguments to cycles with overlapping simplices.

\section{Retracting chains to codimension zero submanifolds}

3A. Definitions. The results of this section are essentially all due to Gromov, but we follow mainly our exposition in [Kuessner 2010]. We start with some recollections about multicomplexes; for details, see [Gromov 1982, Section 3; Kuessner 2010, Section 1].

A multicomplex $K$ is a topological space $|K|$ with a decomposition into simplices, where each $n$-simplex is attached to the $(n-1)$-skeleton $K_{n-1}$ by a simplicial homeomorphism $f: \partial \Delta^{n} \rightarrow K_{n-1}$. (In particular, each $n$-simplex has $n+1$ distinct vertices.) In contrast with simplicial complexes, in a multicomplex there may be $n$-simplices with the same $(n-1)$-skeleton.

We call a multicomplex minimally complete if the following condition holds: Let $\sigma: \Delta^{n} \rightarrow|K|$ be a singular $n$-simplex such that $\partial_{0} \sigma, \ldots, \partial_{n} \sigma$ are distinct simplices of $K$. Then $\sigma$ is homotopic relative $\partial \Delta^{n}$ to a unique simplex in $K$.

We call a minimally complete multicomplex $K$ aspherical if all simplices $\sigma \neq \tau$ in $K$ satisfy $\sigma_{1} \neq \tau_{1}$. That means that simplices are uniquely determined by their 1-skeleton.

Orientations of multicomplexes are defined as usual in simplicial theory. If $\sigma$ is a simplex, $\bar{\sigma}$ will denote the simplex with the opposite orientation.

A submulticomplex $L$ of a multicomplex $K$ is a subset of the set of simplices closed under face maps. $(K, L)$ is a pair of multicomplexes if $K$ is a multicomplex and $L$ is a submulticomplex of $K$.

A group $G$ acts simplicially on a pair of multicomplexes $(K, L)$ if it acts on the set of simplices of $K$, mapping simplices in $L$ to simplices in $L$, so that the action commutes with all face maps. For $g \in G$ and $\sigma$ a simplex in $K$, we denote by $g \sigma$ the simplex obtained by this action. 
3B. Construction of $\boldsymbol{K}(\boldsymbol{X})$. We recall the construction from [Kuessner 2010, Section 1.3] (originally found in [Gromov 1982, pp. 45-46]).

For a topological space $X$, we denote by $S_{*}(X)$ the simplicial set of all singular simplices in $X$ and by $\left|S_{*}(X)\right|$ its geometric realization.

For a topological space $X$, a multicomplex $\widehat{K}(X) \subset\left|S_{*}(X)\right|$ is constructed as follows. The 0 -skeleton $\widehat{K}_{0}(X)$ equals $S_{0}(X)$. The 1 -skeleton $\widehat{K}_{1}(X)$ contains one element in each homotopy class (rel $\{0,1\}$ ) of singular 1 -simplices $f:[0,1] \rightarrow X$ with $f(0) \neq f(1)$. For $n \geq 2$, assuming by recursion that the $(n-1)$-skeleton is defined, the $n$-skeleton $\widehat{K}_{n}(X)$ contains one singular $n$-simplex in each homotopy class (rel boundary) of singular $n$-simplices $f: \Delta^{n} \rightarrow X$ with $\partial f \in \widehat{K}_{n-1}(X)$. We can choose $\widehat{K}(X)$ with the property that $\sigma \in \widehat{K}(X) \Longleftrightarrow \bar{\sigma} \in \widehat{K}(X)$ (recall that the bar denotes orientation reversal). We will henceforth assume that $\widehat{K}(X)$ is constructed according to this condition.

According to [Gromov 1982], $|\widehat{K}(X)|$ is weakly homotopy equivalent to $X$.

The multicomplex $K(X)$ is defined as the quotient

$$
K(X):=\widehat{K}(X) / \sim
$$

where simplices in $\widehat{K}(X)$ are identified if and only if they have the same 1-skeleton. Let $p$ be the canonical projection $p: \widehat{K}(X) \rightarrow K(X)$.

$K(X)$ is minimally complete and aspherical.

If $X^{\prime} \subset X$ is a subspace, we have (not necessarily injective) simplicial mappings $\hat{j}: \widehat{K}\left(X^{\prime}\right) \rightarrow \widehat{K}(X)$ and $j: K\left(X^{\prime}\right) \rightarrow K(X)$.

If $\pi_{1} X^{\prime} \rightarrow \pi_{1} X$ is injective (for each path-connected component of $X^{\prime}$ ), then $j$ is injective ([Kuessner 2010], Section 1.3) and we can (and will) consider $K\left(X^{\prime}\right)$ as a submulticomplex of $K(X)$. (Since simplices in $\widehat{K}\left(X^{\prime}\right)$ have image in $X^{\prime}$, this means that we assume we have constructed $\widehat{K}(X)$ so that simplices in $\widehat{K}(X)$ have image in $X^{\prime}$ whenever this is possible.) If moreover $\pi_{n} X^{\prime} \rightarrow \pi_{n} X$ is injective for all $n \geq 2$ (say, if $X^{\prime}$ is aspherical), then $\hat{j}$ is also injective and $\widehat{K}\left(X^{\prime}\right)$ can be considered as a submulticomplex of $\widehat{K}(X)$.

In particular, if $X$ and $X^{\prime}$ are aspherical and $\pi_{1} X^{\prime} \rightarrow \pi_{1} X$ is injective, there is an inclusion

$$
i_{*}: C_{*}^{\text {simp }}\left(K(X), K\left(X^{\prime}\right)\right)=C_{*}^{\text {simp }}\left(\widehat{K}(X), \widehat{K}\left(X^{\prime}\right)\right) \rightarrow C_{*}^{\text {sing }}\left(X, X^{\prime}\right)
$$

into the relative singular chain complex of $\left(X, X^{\prime}\right)$.

3C. Infinite and locally finite chains. In this paper we will also work with infinite chains, and in particular with locally finite chains on noncompact manifolds, as introduced in [Gromov 1982, Section 0.2].

For a topological space $X$, a formal sum $\sum_{i \in I} a_{i} \sigma_{i}$ of singular $k$-simplices with real coefficients (with a possibly infinite index set $I$, and the convention $a_{i} \neq 0$ 
for $i \in I$ ) is an infinite singular $k$-chain. It is said to be a locally finite chain if each point of $X$ is contained in the image of at most finitely many $\sigma_{i}$. Infinite $k$ chains form a real vector space denoted by $C_{k}^{\inf }(X)$, and locally finite $k$-chains one denoted by $C_{k}^{\mathrm{lf}}(X)$. The boundary operator maps locally finite $k$-chains to locally finite $(k-1)$-chains, hence, for a pair of spaces $\left(X, X^{\prime}\right)$ the homology $H_{*}^{\mathrm{lf}}\left(X, X^{\prime}\right)$ of the complex of locally finite chains can be defined.

For a noncompact, orientable $n$-manifold $X$ with (possibly noncompact) boundary $\partial X$, one has a fundamental class $[X, \partial X] \in H_{n}^{\mathrm{lf}}(X, \partial X)$. We will say that an infinite chain $\sum_{i \in I} a_{i} \sigma_{i}$ represents $[X, \partial X]$ if it is homologous to a locally finite chain representing $[X, \partial X] \in H_{n}^{\mathrm{lf}}(X, \partial X)$.

For a simplicial complex $K$, we denote by $C_{k}^{\text {simp,inf }}(K)$ the $\mathbb{R}$-vector space of (possibly infinite) formal sums $\sum_{i \in I} a_{i} \sigma_{i}$ with $a_{i} \in \mathbb{R}$ and $\sigma_{i} k$-simplices in $K$. If $\pi_{n} X^{\prime} \rightarrow \pi_{n} X$ is injective for $n \geq 1$, we have again the obvious inclusion $i_{*}$ : $C_{*}^{\text {simp,inf }}\left(\widehat{K}(X), \widehat{K}\left(X^{\prime}\right)\right) \rightarrow C_{*}^{\inf }\left(X, X^{\prime}\right)$.

The following observation is of course a well-known application of the homotopy extension property, but we will use it so often that we state it here for reference.

Observation 3.1. Let $X$ be a topological space and $\sigma_{0}: \Delta^{n} \rightarrow X$ a singular simplex. Let $H: \partial \Delta^{n} \times I \rightarrow X$ be a homotopy with $H(x, 0)=\sigma_{0}(x)$ for all $x \in \partial \Delta^{n}$. Then there exists a homotopy $\bar{H}: \Delta^{n} \times I \rightarrow X$ with $\left.\bar{H}\right|_{\partial \Delta^{n} \times I}=H$ and $\left.\bar{H}\right|_{\Delta^{n} \times\{0\}}=\sigma_{0}$.

If $X^{\prime} \subset X$ is a subspace and the images of $\sigma_{0}$ and $H$ belong to $X^{\prime}$, we can choose $\bar{H}$ so that its image belongs to $X^{\prime}$.

Lemma 3.2. Let $\left(X, X^{\prime}\right)$ be a pair of topological spaces. Assume $\pi_{n} X^{\prime} \rightarrow \pi_{n} X$ is injective for each path component of $X^{\prime}$ and each $n \geq 1$.

(a) Let $\sum_{i \in I} a_{i} \tau_{i} \in C_{n}^{\mathrm{inf}}\left(X, X^{\prime}\right)$ be a (possibly infinite) singular $n$-chain. Assume that $I$ is countable, and that each path component of $X$ and each nonempty path component of $X^{\prime}$ contain uncountably many points. Then $\sum_{i \in I} a_{i} \tau_{i}$ is homotopic to a (possibly infinite) simplicial chain

$$
\sum_{i \in I} a_{i} \tau_{i}^{\prime} \in C_{n}^{\operatorname{simp}, \inf }\left(\widehat{K}(X), \widehat{K}\left(X^{\prime}\right)\right) \subset C_{*}^{\inf }\left(X, X^{\prime}\right) .
$$

In particular, $\sum_{i \in I} a_{i} \tau_{i}^{\prime}$ is homologous to $\sum_{i \in I} a_{i} \tau_{i}$.

(b) Let $\sigma_{0} \in \widehat{K}(X)$ and $H: \Delta^{n} \times[0,1] \rightarrow X$ a homotopy with $H(\cdot, 0)=\sigma_{0}$. Consider a minimal triangulation $\Delta^{n} \times[0,1]=\Delta_{0} \cup \ldots \Delta_{n}$ of $\Delta^{n} \times[0,1]$ into $n+1(n+1)$-simplices. Assume that $H\left(\partial \Delta^{n} \times[0,1]\right)$ consists of simplices in $\widehat{K}(X)$. Then $H$ is homotopic (rel $\left.\Delta^{n} \times\{0\} \cup \partial \Delta^{n} \times[0,1]\right)$ to a map $\bar{H}$ : $\Delta^{n} \times[0,1] \rightarrow X$ such that $\left.\bar{H}\right|_{\Delta_{i}} \in \widehat{K}(X)$; in particular $\sigma_{1}:=\bar{H}(\cdot, 1) \in \widehat{K}(X)$.

Proof. (a) From the assumptions it follows that there exists a homotopy of the 0 -skeleton such that each vertex is moved into a distinct point of $X$, and such 
that vertices in $X^{\prime}$ remain in $X^{\prime}$ during the homotopy. By Observation 3.1, this homotopy can by induction be extended to a homotopy of the whole chain.

Now we prove the claim by induction on $k(0 \leq k<n)$. We assume that the $k$-skeleton of $\sum_{i \in I} a_{i} \tau_{i}$ consists of simplices in $\widehat{K}(X)$ and we want to homotope $\sum_{i \in I} a_{i} \tau_{i}$ such that the homotoped $(k+1)$-skeleton consists of simplices in $\widehat{K}(X)$.

By construction, each singular $(k+1)$-simplex $\sigma$ in $X$ with boundary a simplex in $\widehat{K}(X)$ is homotopic (rel boundary) to a unique $(k+1)$-simplex in $\widehat{K}(X)$. Since the homotopy keeps the boundary fixed, the homotopies of different $(k+1)$ simplices are compatible. By Observation 3.1, the homotopy of the $(k+1)$-skeleton can by induction be extended to a homotopy of the whole chain.

If the image of the $(k+1)$-simplex $\sigma$ is contained in $X^{\prime}$, then it is homotopic rel boundary to a simplex in $\widehat{K}\left(X^{\prime}\right)$, for a homotopy with image in $X^{\prime}$. Thus we can realize the homotopy in such a way that all simplices with image in $X^{\prime}$ are homotoped inside $X^{\prime}$.

(b) follows by the same argument as (a), successively applied to $\Delta_{0}, \ldots, \Delta_{n}$.

We remark that there exists a canonical simplicial map

$$
p: C_{*}^{\text {simp,inf }}\left(\widehat{K}(X), \widehat{K}\left(X^{\prime}\right)\right) \rightarrow C_{*}^{\text {simp, inf }}\left(K(X), K\left(X^{\prime}\right)\right),
$$

defined by induction. It is defined to be the identity on the 1-skeleton. If it is defined on the (n-1)-skeleton, for $n \geq 2$, then, for an $n$-simplex $\tau, p(\tau) \in K(X)$ is the unique simplex with $\partial_{i} p(\tau)=p\left(\partial_{i} \tau\right)$ for $i=0, \ldots, n$.

3D. Action of $\boldsymbol{G}=\Pi(\boldsymbol{A})$. We repeat the definitions from [Kuessner 2010, Section 1.5] (originally due to Gromov), as they will be frequently used in the remainder of the paper.

Let $(P, A)$ be a pair of minimally complete multicomplexes. We define its space of nontrivial loops $\Omega^{*} A$ as the set of homotopy classes (rel $\{0,1\}$ ) of continuous maps $\gamma:[0,1] \rightarrow|A|$ with $\gamma(0)=\gamma(1)$ and not homotopic (rel $\{0,1\})$ to a constant map.

We define

$$
\begin{array}{r}
\Pi(A):=\left\{\left\{\gamma_{1}, \ldots, \gamma_{n}\right\}: n \in \mathbb{N}, \gamma_{1}, \ldots, \gamma_{n} \in A_{1} \cup \Omega^{*} A, \gamma_{i}(0)=\gamma_{i}(1) \text { for all } i,\right. \\
\left.\gamma_{i}(0) \neq \gamma_{j}(0), \gamma_{i}(1) \neq \gamma_{j}(1) \text { for } i \neq j\right\} .
\end{array}
$$

If $\gamma, \gamma^{\prime}$ are elements of $A_{1}$ with $\gamma^{\prime} \neq \bar{\gamma}$ and $\gamma(0)=\gamma^{\prime}(1)$, we denote by $\gamma * \gamma^{\prime} \in A_{1}$ the unique edge of $A$ in the homotopy class of the concatenation. ${ }^{1}$ If $\gamma \in A_{1}$ and $\gamma^{\prime} \in \Omega^{*} A$ (or vice versa), with $\gamma(1) \neq \gamma(0)=\gamma^{\prime}(1)=\gamma^{\prime}(0)$, we also denote

\footnotetext{
${ }^{1}$ We follow the usual convention of defining the concatenation of paths by $\gamma * \gamma^{\prime}(t)=\gamma(2 t)$ if $t \leq \frac{1}{2}$ and $\gamma * \gamma^{\prime}(t)=\gamma^{\prime}(2 t-1)$ if $t \geq \frac{1}{2}$. Unfortunately this implies that, in order for $\Pi(A)$ to act on $P$, we will need the multiplication in $\Pi(A)$ to satisfy, for example, $\{\gamma\}\left\{\gamma^{\prime}\right\}=\left\{\gamma^{\prime} * \gamma\right\}$. We hope that this does not lead to confusion.
} 
by $\gamma * \gamma^{\prime} \in A_{1}$ the unique edge in the homotopy class of the concatenation. If $\gamma, \gamma^{\prime} \in \Omega^{*} A$ with $\gamma(1)=\gamma(0)=\gamma^{\prime}(1)=\gamma^{\prime}(0)$, we denote by $\gamma * \gamma^{\prime} \in \Omega^{*} A$ the concatenation of homotopy classes of loops.

We then define a multiplication on $\Pi(A)$ as follows: Given $\left\{\gamma_{1}, \ldots, \gamma_{m}\right\}$ and $\left\{\gamma_{1}^{\prime}, \ldots, \gamma_{n}^{\prime}\right\}$, we reindex the unordered sets $\left\{\gamma_{1}, \ldots, \gamma_{m}\right\}$ and $\left\{\gamma_{1}^{\prime}, \ldots, \gamma_{n}^{\prime}\right\}$ so that $\gamma_{j}(1)=\gamma_{j}^{\prime}(0)$ for $1 \leq j \leq i$ and $\gamma_{j}(1) \neq \gamma_{k}^{\prime}(0)$ for $j \geq i+1$ and $k \geq i+1$. (Since we are assuming that all $\gamma_{j}(1)$ are pairwise distinct, and also all $\gamma_{j}^{\prime}(0)$ are pairwise distinct, such a reindexing exists for some $i \geq 0$, and it is unique up to permuting the indices $\leq i$ and permuting separately the indices of the $\gamma_{j}$ and $\gamma_{k}^{\prime}$ with $j \geq i+1$ and $k \geq i+1$.) Moreover we permute the indices $\{1, \ldots, i\}$ so that there exists some $h$ with $0 \leq h \leq i$ satisfying the following conditions:

- For $1 \leq j \leq h$ we have either $\gamma_{j}^{\prime} \neq \bar{\gamma}_{j} \in A_{1}$ or $\gamma_{j}^{\prime} \neq \gamma_{j}^{-1} \in \Omega^{*} A$.

- For $h<j \leq i$ we have either $\gamma_{j}^{\prime}=\bar{\gamma}_{j} \in A_{1}$ or $\gamma_{j}^{\prime}=\gamma_{j}^{-1} \in \Omega^{*} A$.

With this fixed reindexing we define

$$
\left\{\gamma_{1}, \ldots, \gamma_{m}\right\}\left\{\gamma_{1}^{\prime}, \ldots, \gamma_{n}^{\prime}\right\}:=\left\{\gamma_{1}^{\prime} * \gamma_{1}, \ldots, \gamma_{h}^{\prime} * \gamma_{h}, \gamma_{i+1}, \ldots, \gamma_{m}, \gamma_{i+1}^{\prime}, \ldots, \gamma_{n}^{\prime}\right\}
$$

(Note that we have omitted all $\gamma_{j}^{\prime} * \gamma_{j}$ with $j>h$. The choice of $\gamma_{j}^{\prime} * \gamma_{j}$ rather than $\gamma_{j} * \gamma_{j}^{\prime}$ is just because we want to define a left action on $(P, A)$.)

We have shown in [Kuessner 2010] (footnote to Section 1.5.1) that the product belongs to $\Pi(A)$. Moreover, the multiplication so defined is independent of the chosen reindexing. It is clearly associative. A neutral element is given by the empty set. The inverse to $\left\{\gamma_{1}, \ldots, \gamma_{n}\right\}$ is given by $\left\{\gamma_{1}^{\prime}, \ldots, \gamma_{n}^{\prime}\right\}$, with $\gamma_{i}^{\prime}=\bar{\gamma}_{i}$ if $\gamma_{i} \in A_{1}$ and $\gamma_{i}^{\prime}=\gamma_{i}^{-1}$ if $\gamma_{i} \in \Omega^{*} A$. (Indeed, in this case $h=0$; thus $\left\{\gamma_{1}, \ldots, \gamma_{n}\right\}\left\{\gamma_{1}^{\prime}, \ldots, \gamma_{n}^{\prime}\right\}$ is the empty set.) Thus we have defined a group law on $\Pi(A)$.

Remark. There is an inclusion

$$
\Pi(A) \subset \operatorname{map}_{0}\left(A_{0},[[0,1],|A|]_{|P|}\right),
$$

where $[[0,1],|A|]_{|P|}$ is the set of homotopy classes (in $|P|$ ) rel $\{0,1\}$ of maps from $[0,1]$ to $|A|$, and $\operatorname{map}_{0}\left(A_{0},[[0,1],|A|]_{|P|}\right)$ is the set of maps $f: A_{0} \rightarrow$ $[[0,1],|A|]_{|P|}$ with

- $f(y)(0)=y$ for all $y \in A_{0}$, and

$-f(\cdot)(1): A_{0} \rightarrow A_{0}$ is a bijection.

This inclusion is given by sending $\left\{\gamma_{1}, \ldots, \gamma_{n}\right\}$ to the map $f$ defined by $f\left(\gamma_{i}(0)\right)=$ $\left[\gamma_{i}\right]$ for $i=1, \ldots, n$, and $f(y)=\left[c_{y}\right]$ (the constant path) for $y \notin\left\{\gamma_{1}(0), \ldots, \gamma_{n}(0)\right\}$. The inclusion is a homomorphism with respect to the group law defined by

$$
[g f(y)]:=[f(y)] *[g(f(y)(1))]
$$

on $\operatorname{map}_{0}\left(A_{0},[[0,1],|A|]_{|P|}\right)$. 
3E. Action of $\Pi(A)$ on $P$. From now on we assume that $P$ is aspherical. We define an action of $\operatorname{map}_{0}\left(A_{0},[[0,1],|A|]_{|P|}\right)$ on $P$. This gives an action of $\Pi(A)$ on $P$.

Let $g \in \operatorname{map}_{0}\left(A_{0},[(0,1),|A|]_{|P|}\right)$. Define $g y=g(y)(1)$ for $y \in A_{0}$ and $g x=x$ for $x \in P_{0}-A_{0}$. This defines the action on the 0 -skeleton of $P$.

We extend this to an action on the 1 -skeleton of $P$. Recall that, by minimal completeness of $P, 1$-simplices $\sigma$ are in one-to-one correspondence with homotopy classes (rel $\{0,1\}$ ) of (nonclosed) singular 1-simplices in $|P|$ with vertices in $P_{0}$. Using this correspondence, define

$$
g \sigma:=[\overline{g(\sigma(0))}] *[\sigma] *[g(\sigma(1))],
$$

where $*$ denotes concatenation of (homotopy classes of) paths.

In [Kuessner 2010, Section 1.5.1] we proved that this defines an action on $P_{1}$ and that there is an extension of ths action to an action on $P$. (The extension is unique because $P$ is aspherical.)

We remark, because this will be one of the assumptions to apply Lemma 3.7, that the action of any element $g \in \Pi(A)$ is homotopic to the identity. The homotopy between the action of the identity and the action of $\left\{\gamma_{1}, \ldots, \gamma_{r}\right\}$ given by the action of $\left\{\gamma_{1}^{t}, \ldots, \gamma_{r}^{t}\right\}, 0 \leq t \leq 1$, with $\gamma_{i}^{t}(s)=\gamma_{i}(s t)$.

The next lemma follows directly from the construction, but we will use it so often that we want to explicitly state it.

Lemma 3.3. Let $(P, A)$ be a pair of aspherical, minimally complete multicomplexes, with the action of $G=\Pi(A)$. If $\sigma \in P$ is a simplex all of whose vertices are not in $A$, then $g \sigma=\sigma$ for all $g \in G$.

For a topological space and a subset $P \subset S_{*}(X)$ closed under face maps, the (antisymmetric) bounded cohomology $H_{b}^{*}(P)$ and its pseudonorm are defined literally like for multicomplexes in [Gromov 1982, Section 3.2]. The following well-known fact will be needed for applications of Lemma 3.7 (to the setting of Theorem 1.1) with $P=K^{\mathrm{str}}(\partial Q), G=\Pi\left(K\left(\partial_{0} Q\right)\right)$.

Lemma 3.4. (a) Let $(P, A)$ be a pair of minimally complete multicomplexes. If each connected component of $|A|$ has amenable fundamental group, then $\Pi(A)$ is amenable.

(b) Let $X$ be a topological space, $P \subset S_{*}(X)$ a subset closed under face maps, and $G$ an amenable group acting on $P$. Then the canonical homomorphism

$$
\operatorname{id} \otimes 1: C_{*}^{\text {simp }}(P) \rightarrow C_{*}^{\text {simp }}(P) \otimes_{\mathbb{Z} G} \mathbb{Z}
$$

induces an isometric monomorphism in bounded cohomology.

The proof of (a) is an obvious adaptation of that of [Kuessner 2010, Lemma 4]. Part (b) is proved by averaging bounded cochains; see [Gromov 1982]. 


\section{F. Retraction to central simplices.}

Lemma 3.5. Let $(N, \partial N)$ be a pair of topological spaces with $N=Q \cup R$ for two subspaces $Q, R$. Let

$\partial_{0} Q=Q \cap R, \partial_{1} Q=Q \cap \partial N, \partial_{1} R=R \cap \partial N, \partial Q=\partial_{0} Q \cup \partial_{1} Q, \partial R=\partial_{0} Q \cup \partial_{1} R$.

Assume that $\partial_{1} Q \rightarrow Q \rightarrow N, \partial_{1} R \rightarrow R \rightarrow N, \partial N \rightarrow N, \partial_{0} Q \rightarrow Q, \partial_{0} Q \rightarrow R$ are $\pi_{1}$-injective, and that $\partial N, \partial_{1} Q, \partial_{1} R, \partial_{0} Q$ are aspherical (so the corresponding $K(\cdot)$ can be considered as submulticomplexes of $K(N))$.

In connection with the simplicial action of $G=\Pi\left(K\left(\partial_{0} Q\right)\right)$ on $K(N)$, there is a chain homomorphism

$$
r: C_{*}^{\text {simp,inf }}(K(N)) \otimes_{\mathbb{Z} G} \mathbb{Z} \rightarrow C_{*}^{\text {simp,inf }}(K(Q)) \otimes_{\mathbb{Z} G} \mathbb{Z}
$$

in degrees $* \geq 2$, mapping $C_{*}^{\text {simp, inf }}(G K(\partial N)) \otimes_{\mathbb{Z} G} \mathbb{Z}$ to $C_{*}^{\text {simp, inf }}\left(G K\left(\partial_{1} Q\right)\right) \otimes_{\mathbb{Z} G} \mathbb{Z}$, and such that

- if $\sigma$ is a simplex in $K(N)$, then $r(\sigma \otimes 1)=\kappa \otimes 1$, where either $\kappa$ is a simplex in $K(Q)$ or $\kappa=0$;

- if $\sigma$ is a simplex in $K(Q)$, then $r(\sigma \otimes 1)=\sigma \otimes 1$;

- if $\sigma$ is a simplex in $K(R)$, then $r(\sigma \otimes 1)=0$.

Proof. This is [Kuessner 2010, Proposition 6]. (We have replaced the assumption $\operatorname{ker}\left(\pi_{1} \partial_{0} Q \rightarrow \pi_{1} Q\right)=\operatorname{ker}\left(\pi_{1} \partial_{0} Q \rightarrow \pi_{1} R\right)$ from that reference by the stronger assumption of $\pi_{1}$-injectivity, since this will be true in all our applications and we have no need for the more general assumption.) The conclusion is stated in [Kuessner 2010] for locally finite chains, but of course $r$ extends linearly to infinite chains.

Remark. If some edge of $\sigma$ is contained in $K\left(\partial_{0} Q\right)=K(Q) \cap K(R)$, then

$$
\sigma \otimes 1=0 \in C_{*}^{\operatorname{simp}, \text { inf }}(K(N)) \otimes_{\mathbb{Z} G} \mathbb{Z} ;
$$

see [Kuessner 2010, Section 1.5.2]. (The proof is essentially the same as that of Lemma 5.17 below.) In particular, if $\sigma$ is contained in both $K(Q)$ and $K(R)$, then $r(\sigma \otimes 1)=r(0)=0$.

3G. Fundamental cycles in $K(N)$ and $K(Q)$. Let $N$ be a (possibly noncompact) connected, orientable $n$-manifold with (possibly noncompact) boundary $\partial N$. Then $H_{n}^{\mathrm{lf}}(N, \partial N) \simeq \mathbb{Z}$ by Whitehead's theorem and a generator is called $[N, \partial N]$. (It is only defined up to sign, but this will not concern our arguments.) Recall that an infinite chain is said to represent $[N, \partial N]$ if it is homologous to a locally finite chain representing $[N, \partial N]$. 
If $\partial N \rightarrow N$ is $\pi_{1}$-injective and $\partial N$ is aspherical, we know from Section $3 \mathrm{~B}$ that

$$
C_{*}^{\text {simp,inf }}(\widehat{K}(N), \widehat{K}(\partial N)) \subset C_{*}^{\operatorname{sing}, \inf }(N, \partial N) .
$$

Thus it makes sense to say that some chain $z \in C_{*}^{\operatorname{simp}, \text { inf }}(\widehat{K}(N), \widehat{K}(\partial N))$ represents the fundamental class $[N, \partial N]$.

If $\partial_{1} Q \rightarrow Q$ is $\pi_{1}$-injective and $Q$ and $\partial_{1} Q$ are aspherical, and if we set $G:=$ $\Pi\left(K\left(\partial_{0} Q\right)\right)$, then $C_{*}^{\text {simp,inf }}\left(G K\left(\partial_{1} Q\right)\right)=C_{*}^{\text {simp,inf }}\left(G \widehat{K}\left(\partial_{1} Q\right)\right) \subset C_{*}^{\text {sing,inf }}(\partial Q)$, as $G$ maps simplices in $\operatorname{im}(K(\partial Q) \rightarrow K(Q))$ to simplices in $\operatorname{im}(K(\partial Q) \rightarrow K(Q))$. Thus it makes sense to say that some chain $z \in C_{*}^{\text {simp,inf }}\left(K(Q), G K\left(\partial_{1} Q\right)\right)$ represents the fundamental class $[Q, \partial Q]$.

The projection $p: \widehat{K}(N) \rightarrow K(N)$ is defined at the end of Section 3B.

Lemma 3.6. Let $N$ be an orientable $n$-manifold with boundary (where $n \geq 2$ ), and let $Q, R \subset N$ be orientable n-manifolds with boundary such that $N=Q \cup R$ satisfies the assumptions of Lemma 3.5 and that $\partial_{0} Q, \partial_{1} Q \subset \partial Q$ and $\partial_{1} R \subset \partial R$ are $(n-1)$-dimensional submanifolds (with boundary) of $\partial Q$ or $\partial R$. Assume also that $Q$ is aspherical. Let $\sum_{i} a_{i} \sigma_{i} \in C_{n}^{\operatorname{simp}, \inf }(\widehat{K}(N), \widehat{K}(\partial N))$ represent $[N, \partial N]$.

(a) $\sum_{i} a_{i} r\left(p\left(\sigma_{i}\right)\right) \otimes 1 \in C_{n}^{\text {simp, inf }}\left(K(Q), G K\left(\partial_{1} Q\right)\right) \otimes_{\mathbb{Z} G} \mathbb{Z}$ represents $[Q, \partial Q] \otimes 1$.

(b) $\partial \sum_{i} a_{i} r\left(p\left(\sigma_{i}\right)\right) \otimes 1 \in C_{n}^{\text {simp,inf }}(G K(\partial Q)) \otimes_{\mathbb{Z} G} \mathbb{Z}$ represents $[\partial Q] \otimes 1$.

Remark. Explicitly, statement (a) means that the element on the left represents the image of $h \otimes 1$ under the canonical homomorphism $H_{n}^{\text {sing,inf }}(Q, \partial Q) \otimes_{\mathbb{Z} G} \mathbb{Z} \rightarrow$ $H_{n}\left(C_{*}^{\text {sing,inf }}(Q, \partial Q) \otimes_{\mathbb{Z} G} \mathbb{Z}\right)$, where $h \in H_{n}^{\text {simp,inf }}\left(K(Q), G K\left(\partial_{1} Q\right)\right)$ represents $[Q, \partial Q] \in H_{n}^{\text {sing }}(Q, \partial Q)$. Similarly, (b) means that the element represents the image of $h \otimes 1$ under the canonical homomorphism $H_{n}^{\text {simp,inf }}\left(G K\left(\partial_{1} Q\right)\right) \otimes_{\mathbb{Z} G} \mathbb{Z} \rightarrow$ $H_{n}\left(C_{*}^{\text {simp,inf }}\left(G K\left(\partial_{1} Q\right)\right) \otimes_{\mathbb{Z} G} \mathbb{Z}\right)$, where $h \in H_{n}^{\text {simp,inf }}\left(G K\left(\partial_{1} Q\right)\right)$ represents $[\partial Q] \in$ $H_{n}^{\text {sing }}(\partial Q)$.

Proof. Since $p$ and $r$ are chain maps, it suffices to check the claim for some chosen representative of $[N, \partial N]$. So let $z \in C_{*}^{\operatorname{simp}, \text { inf }}(\widehat{K}(N), \widehat{K}(\partial N))$ be a representative of $[N, \partial N]$ chosen so that

$$
p(z)=z_{Q}+z_{R},
$$

where $z_{Q}$ represents $[Q, \partial Q]$ and $z_{R}$ represents $[R, \partial R]$, and so that

$$
\partial z_{Q}=w_{1}+w_{2}, \partial z_{R}=-w_{2}+w_{3}
$$

with $w_{1} \in C_{n-1}^{\text {simp,inf }}\left(K\left(\partial_{1} Q\right)\right)$ representing $\left[\partial_{1} Q\right], w_{2} \in C_{n-1}^{\text {simp,inf }}\left(K\left(\partial_{0} Q\right)\right)$ representing $\left[\partial_{0} Q\right]$, and $w_{3} \in C_{n-1}^{\operatorname{simp}, \text { inf }}\left(K\left(\partial_{1} R\right)\right)$ representing $\left[\partial_{1} R\right]$.

From Lemma 3.5 we have

$$
r(p(z) \otimes 1)=z_{Q} \otimes 1,
$$


which implies the first claim, and

$$
\partial r(p(z) \otimes 1)=\partial z_{Q} \otimes 1=w_{1} \otimes 1+w_{2} \otimes 1 .
$$

Since $w_{1}+w_{2}$ represents $[\partial Q]$, this implies the second claim.

Remark. From the remark after Lemma 3.5 we have $w_{2} \otimes 1=0$. This implies $\partial r(p(z) \otimes 1)=\partial z_{Q} \otimes 1=w_{1} \otimes 1$, that is, $\partial r(p(z) \otimes 1)$ represents at the same time $[\partial Q] \otimes 1$ and $\left[\partial_{1} Q\right] \otimes 1$.

3H. Using amenability. The next lemma is well-known in slightly different formulations and we reprove it here only for completeness. (It has of course a relative version as well, but we will not need that for our argument.)

We will apply ${ }^{2}$ this lemma in the proof of Theorem 1.1 with $X=\partial Q, G=$ $q_{*}\left(\Pi\left(K\left(\partial_{0} Q\right)\right)\right)$ and $K=G K^{\mathrm{str}}\left(\partial_{1} Q\right)$.

Lemma 3.7. Let $X$ be a closed, orientable manifold and $K \subset S_{*}(X)$ closed under face maps. Assume that

- there is an amenable group $G$ acting on $K$, such that the action of each $g \in G$ on $|K|$ is homotopic to the identity, and

- there is a fundamental cycle $z \in C_{*}^{\mathrm{simp}}(K)$ such that $z \otimes 1$ is homologous to a cycle $h=\sum_{j=1}^{s} b_{j} \tau_{j} \otimes 1 \in C_{*}^{\text {simp }}(K) \otimes_{\mathbb{Z} G} \mathbb{Z}$.

Then

$$
\|X\| \leq \sum_{j=1}^{s}\left|b_{j}\right| .
$$

Proof. If $\|X\|=0$, there is nothing to prove. Thus we may assume $\|X\| \neq 0$, which implies [Gromov 1982, p. 17] that there is $\beta \in H_{b}^{n}(X)$, a bounded cohomology class dual to $[X] \in H_{n}(X)$, with $\|\beta\|=1 /\|X\|$.

Let $p: C_{*}^{\text {simp }}(K) \rightarrow C_{*}^{\text {simp }}(K) \otimes_{\mathbb{Z} G} \mathbb{Z}$ be the homomorphism defined by $p(\sigma)=$ $\sigma \otimes 1$. Since $G$ is amenable we have, by the proof of [Gromov 1982, Lemma 4b], an "averaging homomorphism" $A v: H_{b}^{*}(K) \rightarrow H_{b}^{*}\left(C_{*}(K) \otimes_{\mathbb{Z} G} \mathbb{Z}\right)$ such that $A v$ is left-inverse to $p^{*}$ and $A v$ is an isometry. Hence

$$
\|A v(\beta)\|=\|\beta\|=\frac{1}{\|X\|} .
$$

\footnotetext{
${ }^{2}$ If a group $G$ acts simplicially on a multicomplex $M$, then $C_{*}(M) \otimes_{\mathbb{Z} G} \mathbb{Z}$ are abelian groups with well-defined boundary operator $\partial_{*} \otimes 1$, even though $M / G$ may not be a multicomplex, like for the action of $G=\Pi_{X}(X)$ on $K(X)$, for a topological space $X$.

We remark that $C_{*}(M) \otimes_{\mathbb{Z} G} \mathbb{Z} \simeq C_{*}(M) \otimes_{\mathbb{R} G} \mathbb{R}$ is just the quotient chain complex for the $G$ action. In particular, even though $C_{*}(M)$ is an $\mathbb{R} G$-module, it does not make any difference whether we tensor over $\mathbb{Z} G$ or $\mathbb{R} G$.
} 
Moreover, denoting by $\left[\sum_{j=1}^{s} b_{j} \tau_{j} \otimes 1\right]$ the homology class of $\sum_{j=1}^{s} b_{j} \tau_{j} \otimes 1$, we have obviously

$$
\left|A v(\beta)\left[\sum_{j=1}^{s} b_{j} \tau_{j} \otimes 1\right]\right| \leq\|A v(\beta)\| \sum_{j=1}^{s}\left|b_{j}\right|
$$

and therefore

$$
\|X\|=\frac{1}{\|A v(\beta)\|} \leq \frac{\sum_{j=1}^{s}\left|b_{j}\right|}{\left|A v(\beta)\left[\sum_{j=1}^{s} b_{j} \tau_{j} \otimes 1\right]\right|} .
$$

It remains to prove that $A v(\beta)\left[\sum_{j=1}^{s} b_{j} \tau_{j} \otimes 1\right]=1$. For this we have to look at the definition of $A v$, which is as follows:

Let $\gamma \in C_{b}^{*}(K)$ be a bounded cochain. By amenability there exists a bi-invariant mean $a v: B(G) \rightarrow \mathbb{R}$ on the bounded functions on $G$ with $\inf _{g \in G} \delta(g) \leq a v(\delta) \leq$ $\sup _{g \in G} \delta(g)$ for all $\delta \in B(G)$. Then, given any $p(\sigma) \in C_{*}(K) \otimes_{\mathbb{Z} G} \mathbb{Z}$, one can fix an identification between $G$ and $G \sigma$, the set of all $\sigma^{\prime}$ with $p\left(\sigma^{\prime}\right)=p(\sigma)$, and thus consider the restriction of $\gamma$ to $G \sigma$ as a bounded cochain on $G$. Define $A v(\gamma)(p(\sigma))$ to be the average $a v$ of this bounded cochain on $G \simeq G \sigma$. (This definition is independent of all choices; see [Ivanov 1985].)

Now, if $z=\sum_{j=1}^{s} b_{j} \tau_{j}$ is a fundamental cycle, we have $\beta(z)=1$.

If $g \in G$ is arbitrary, then left multiplication with $g$ is a chain map on $C_{*}^{\text {simp }}(K)$, as well as on $C_{*}^{\text {sing }}(X)$. Since the action of $g$ on $|K|$ is homotopic to the identity, it induces the identity on the image of $C_{*}^{\text {simp }}(K) \rightarrow C_{*}^{\text {sing }}(X)$. Thus, for each cycle $z \in C_{*}^{\text {simp }}(K)$ representing $[X] \in H_{*}^{\text {sing }}(X)$, the cycle $g z \in C_{*}^{\text {simp }}(K)$ must also represent $[X]$.

If $g z$ represents $[X]$, then $\beta(g z)=\beta([X])=1$. In conclusion, $\beta\left(p\left(z^{\prime}\right)\right)=1$ for each $z^{\prime}$ with $p\left(z^{\prime}\right)=p(z)$. By the definition of $A v$, this implies $A v(\beta)(p(z))=1$ for each fundamental cycle $z$. In particular, $A v(\beta)\left[\sum_{j=1}^{s} b_{j} \tau_{j} \otimes 1\right]=1$, which finishes the proof of the lemma.

Remark. In the proof of Theorem 1.1, we will work with $C_{*}^{\text {simp }}(K) \otimes_{\mathbb{Z} G} \mathbb{Z}$ rather than $C_{*}^{\text {simp }}(K)$. This is analogous to Agol's construction of "crushing the cusps to

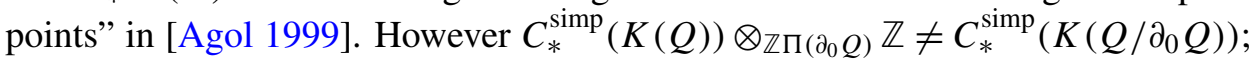
thus one cannot simplify our arguments by working directly with $Q / \partial_{0} Q$.

\section{Disjoint planes in a simplex}

In this section, we will discuss the possibilities for how a simplex can be cut by planes without producing parallel arcs in the boundary. (More precisely, we pose the additional condition that the components of the complement can be colored by black and white such that all vertices belong to black components, and we actually want to avoid only parallel arcs in the boundary of white components.) 
For example, for the 3-simplex, it will follow that there is essentially only the possibility in Case 1 pictured below; meanwhile, in Case 2, each triangle has a parallel arc with another triangle, regardless how the quadrangle is triangulated.
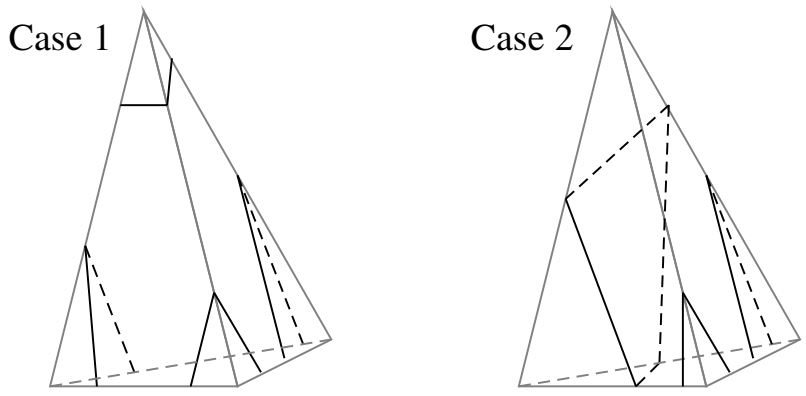

Let $\Delta^{n} \subset \mathbb{R}^{n+1}$ be the standard simplex ${ }^{3}$ with vertices $v_{0}, \ldots, v_{n}$. It is contained in the plane $E=\left\{\left(x_{1}, \ldots, x_{n+1}\right) \in \mathbb{R}^{n+1}: x_{1}+\cdots+x_{n+1}=1\right\}$.

In this section we will be interested in $(n-1)$-dimensional affine planes $P \subset E$ whose intersection with $\Delta^{n}$ either contains no vertex, consists of exactly one vertex, or consists of a face of $\Delta^{n}$. For such planes we define their type as follows.

Definition 4.1. Let $P \subset E$ be an (n-1)-dimensional affine plane such that $P \cap \Delta^{n}$ contains no vertex, consists of exactly one vertex, or consists of a face of $\Delta^{n}$.

- If $P \cap \Delta^{n}=\partial_{0} \Delta^{n}$, we say that $P$ is of type $\{0\}$.

- If $P \cap \Delta^{n}=\partial_{j} \Delta^{n}$ with $j \geq 1$, we say that $P$ is of type $\{01 \ldots \hat{j} \ldots n\}$.

- If $P \cap\left\{v_{0}, \ldots, v_{n}\right\}=\left\{v_{0}\right\}$, we say that $P$ is of type $\{0\}$.

- If $P \cap\left\{v_{0}, \ldots, v_{n}\right\}=\varnothing$ or $P \cap\left\{v_{0}, \ldots, v_{n}\right\}=\left\{v_{j}\right\}$ with $j \geq 1$, we say that $P$ is of type $\left\{0 a_{1} \ldots a_{k}\right\}$ with $a_{1}, \ldots, a_{k} \in\{1, \ldots, n\}$ if the following condition is satisfied: $v_{i}$ belongs to the same connected component of $\Delta^{n}-\left(P \cap \Delta^{n}\right)$ as $v_{0}$ if and only if $i \in\left\{a_{1}, \ldots, a_{k}\right\}$.

Observation 4.2. Let $P_{1}$ be a plane of type $\left\{0 a_{1} \ldots a_{k}\right\}$ and $P_{2}$ a plane of type $\left\{0 b_{1} \ldots b_{l}\right\}$. Assume that $Q_{1}:=P_{1} \cap \Delta^{n} \neq \varnothing$ and $Q_{2}:=P_{2} \cap \Delta^{n} \neq \varnothing$. Then $Q_{1} \cap Q_{2}=\varnothing$ implies that either $\left\{a_{1}, \ldots, a_{k}\right\}=\left\{b_{1}, \ldots, b_{l}\right\}$ or exactly one of the following conditions holds:

$$
\begin{aligned}
& -\left\{a_{1}, \ldots, a_{k}\right\} \subset\left\{b_{1}, \ldots, b_{l}\right\} . \\
& -\left\{b_{1}, \ldots, b_{l}\right\} \subset\left\{a_{1}, \ldots, a_{k}\right\} . \\
& -\left\{a_{1}, \ldots, a_{k}\right\} \cup\left\{b_{1}, \ldots, b_{l}\right\}=\{1, \ldots, n\} .
\end{aligned}
$$

${ }^{3}$ As usual, $v_{i}$ is the vertex with all coordinates except the $i$-th equal to zero, and $\partial_{i} \Delta^{n}$ denotes the subsimplex spanned by all vertices except $v_{i}$. We will occasionally identify singular 1 -simplices $\sigma: \Delta^{1} \rightarrow M$ with paths $e:[0,1] \rightarrow M$ by the rule $e(t)=\sigma(t, 1-t)$. In particular, $e(0)=\sigma\left(v_{0}\right)=\partial_{1} \sigma$ and $e(1)=\sigma\left(v_{1}\right)=\partial_{0} \sigma$. 
Proof. $\Delta^{n}-Q_{1}$ consists of two connected components, $C_{1}$ and $C_{2}$. Similarly, $\Delta^{n}-Q_{2}$ consists of two connected components, $D_{1}$ and $D_{2}$. Choose the numbering so $v_{0} \in C_{1}$ and $v_{0} \in C_{2}$. In particular, $C_{1} \cap D_{1} \neq \varnothing$.

Since $Q_{1} \cap Q_{2}=\varnothing$, it follows that $Q_{2}$ is contained in one of $C_{1}$ or $C_{2}$, and $Q_{1}$ is contained in one of $D_{1}$ or $D_{2}$.

If $Q_{1} \subset D_{1}$, either we have $C_{1} \subset D_{1}$, which implies $\left\{a_{1}, \ldots, a_{k}\right\} \subset\left\{b_{1}, \ldots, b_{l}\right\}$, or we have $C_{2} \subset D_{1}$, which implies $\{1, \ldots, n\}-\left\{a_{1}, \ldots, a_{k}\right\} \subset\left\{b_{1}, \ldots, b_{l}\right\}$, hence $\left\{a_{1}, \ldots, a_{k}\right\} \cup\left\{b_{1}, \ldots, b_{l}\right\}=\{1, \ldots, n\}$.

If instead $Q_{1} \subset D_{2}$, we have $Q_{2} \subset C_{1}$. After interchanging $Q_{1}$ and $Q_{2}$ we are back in the case of the previous paragraph.

Notational remark. Arc will mean the intersection of an (n-1)-dimensional affine plane $P \subset E$ (such that $P \cap \Delta^{n} \neq \varnothing$ either contains no vertex, consists of exactly one vertex or consists of a face) with a 2-dimensional subsimplex $\tau^{2} \subset \Delta^{n}$. If an arc consists of only one vertex, we call it a degenerate arc.

Definition 4.3 (parallel arcs). Let $P_{1}, P_{2} \subset E$ be $(n-1)$-dimensional affine planes. Let $\tau$ be a 2-dimensional subsimplex of $\Delta^{n}$ with vertices $v_{r}, v_{s}, v_{t}$. We say that the disjoint $\operatorname{arcs} e_{1}, e_{2}$ obtained as intersections of $P_{1}$ and $P_{2}$, respectively, with $\tau$ are parallel arcs if one of the following conditions holds:

- Both are nondegenerate and any two of $\left\{v_{r}, v_{s}, v_{t}\right\}$ belong to the same connected component of $\tau-e_{1}$ if and only if they belong to the same connected component of $\tau-e_{2}$.

- One, say $e_{1}$, is nondegenerate, the other, say with vertices $v_{s}, v_{t}$, is contained in a face, and $v_{r}$ does not belong to the same connected component of $\tau-e_{1}$ as either $v_{s}$ and $v_{t}$.

- One, say $e_{1}$, is nondegenerate, the other is degenerate, say equal to $v_{r}$, and $v_{s}, v_{t}$ do not belong to the same connected component of $\tau-e_{1}$ as $v_{r}$.

- Both are degenerate and equal.

- Both are contained in a face and equal.

- One is degenerate, the other is contained in a face.

Lemma 4.4. Let $\Delta^{n} \subset \mathbb{R}^{n+1}$ be the standard simplex. Let $P_{1}, P_{2} \subset E$ be $(n-1)$ dimensional affine planes with $Q_{i}=P_{i} \cap \Delta^{n} \neq \varnothing$ for $i=1,2$. Let $P_{1}$ be of type $\left\{0 a_{1} \ldots a_{k}\right\}$ with $1 \leq k \leq n-2$ and $P_{2}$ of type $\left\{0 b_{1} \ldots b_{l}\right\}$ with l arbitrary. Then either $Q_{1} \cap Q_{2} \neq \varnothing$, or $Q_{1}$ and $Q_{2}$ have a parallel arc.

Proof. Assume that $Q_{1} \cap Q_{2}=\varnothing$. By Observation 4.2, there are four possible cases:

$-\left\{0 a_{1} \ldots a_{k}\right\}=\left\{0 b_{1} \ldots b_{l}\right\}$. Then we clearly have parallel arcs. 
- $\left\{0 a_{1} \ldots a_{k}\right\}$ is a proper subset of $\left\{0 b_{1} \ldots b_{l}\right\}$, i.e., $1 \leq k<l \leq n-1$ and $a_{1}=b_{1}, \ldots, a_{k}=b_{k}$. There is at least one index, say $i$, not contained in $\left\{0 b_{1} \ldots b_{l}\right\}$. The 2-dimensional subsimplex with vertices $v_{0}, v_{a_{1}}, v_{i}$ intersects $P_{1}$ and $P_{2}$ in parallel arcs, because $P_{1}$ and $P_{2}$ both separate $v_{0}$ and $v_{a_{k}}$ from $v_{i}$.

- $\left\{0 b_{1} \ldots b_{l}\right\}$ is a proper subset of $\left\{0 a_{1} \ldots a_{k}\right\}$, i.e., $0 \leq l<k \leq n-2$ and $a_{1}=b_{1}, \ldots, a_{l}=b_{l}$. There are two indices $i, j$ not contained in $\left\{0 a_{1} \ldots a_{k}\right\}$. The 2-dimensional subsimplex with vertices $v_{0}, v_{i}, v_{j}$ intersects $P_{1}$ and $P_{2}$ in parallel arcs, because $P_{1}$ and $P_{2}$ both separate $v_{0}$ from $v_{i}$ and $v_{j}$.

$-\left\{a_{1}, \ldots, a_{k}\right\} \cup\left\{b_{1}, \ldots, b_{l}\right\}=\{1, \ldots, n\}$. Since $k \leq n-2$, there are two indices $i, j$ not contained in $\in\left\{0 a_{1} \ldots a_{k}\right\}$. Hence $i, j \in\left\{b_{1}, \ldots, b_{l}\right\}$. There exists an index $h \in\left\{a_{1}, \ldots, a_{k}\right\}$ such that $h \notin\left\{b_{1}, \ldots, b_{l}\right\}$; otherwise, we would have $\left\{a_{1}, \ldots, a_{k}\right\} \subset\left\{b_{1}, \ldots, b_{l}\right\}$, hence $\{1, \ldots, n\}=\left\{a_{1}, \ldots, a_{k}\right\} \cup\left\{b_{1}, \ldots, b_{l}\right\} \subset$ $\left\{b_{1}, \ldots, b_{l}\right\}$, contradicting $Q_{2} \neq \varnothing$. Now the 2-dimensional subsimplex with vertices $v_{i}, v_{j}, v_{h}$ intersects $P_{1}$ and $P_{2}$ in parallel arcs, because both $P_{1}$ and $P_{2}$ separate $v_{i}$ and $v_{j}$ from $v_{h}$.

Definition 4.5 (canonical coloring of complementary regions). Let $P_{1}, P_{2}, \ldots \subset E$ be a (possibly infinite) set of (n-1)-dimensional affine planes with $Q_{i}:=P_{i} \cap \Delta^{n}$ nonempty and $Q_{i} \cap Q_{j}=\varnothing$ for all $i \neq j$. Assume that each $Q_{i}$ either contains no vertices or consists of exactly one vertex.

A coloring of the connected components of $\Delta^{n}-\bigcup_{i} Q_{i}$ by the colors black and white, and of all the $Q_{i}$ by black, is called a canonical coloring (associated to $P_{1}, P_{2}, \ldots$ ) if all the vertices of $\Delta^{n}$ are colored black and each $Q_{i}$ is incident to at least one white component.

Definition 4.6 (white-parallel arcs). Let $\left\{P_{i}: i \in I\right\}$ be a set of (n-1)-dimensional affine planes $P_{i} \subset E$, with $Q_{i}:=P_{i} \cap \Delta^{n} \neq \varnothing$ for $i \in I$. Assume that $Q_{i} \cap Q_{j}=\varnothing$ for all $i \neq j \in I$, and that we have a canonical coloring associated to $\left\{P_{i}: i \in I\right\}$. We say that $\operatorname{arcs} e_{i}, e_{j}$ obtained as intersections of $P_{i}, P_{j}(i, j \in I)$ with some 2dimensional subsimplex of $\Delta^{n}$ are white-parallel arcs if they are parallel arcs and belong to the boundary of the closure of the same white component.

We mention two consequences of Lemma 4.4. They will not be needed for the proof of Lemma 4.13, but they will be necessary for the proof of Theorem 1.1.

Corollary 4.7. Let $\Delta^{n} \subset \mathbb{R}^{n+1}$ be the standard simplex. Let $P_{1}, \ldots, P_{m} \subset E$ be a finite set of (n-1)-dimensional affine planes and let $Q_{i}=P_{i} \cap \Delta^{n}$ for $i=1, \ldots, m$. Assume that $Q_{i} \cap Q_{j}=\varnothing$ for all $i \neq j$, and that we have an associated canonical coloring such that $Q_{i}$ and $Q_{j}$ do not have a white-parallel arc for $i \neq j$.

Then, unless $m=0$, we have $m=n+1$ and $P_{1}$ is of type $\{0\}, P_{n+1}$ is of type $\{01 \ldots n-1\}$, and $P_{i}$ is of type $\{01 \ldots \widehat{i-1} \ldots n\}$ for $i=2, \ldots, n$. 
Proof. If the conclusion were not true, there would exist a plane $P_{1}$ of type $\left\{0 a_{1} \ldots a_{k}\right\}$ with $1 \leq k \leq n-2$. Let $W$ be the white component of the canonical coloring that is incident to $P_{1}$. Because, for a canonical coloring, no vertex belongs to a white component, there must be at least one more plane $P_{2}$ incident to $W$. Since $Q_{1} \cap Q_{2}=\varnothing$, from Lemma 4.4 we get that $Q_{1}$ and $Q_{2}$ have a parallel arc. Because $Q_{1}$ and $Q_{2}$ are incident to $W$, the arc is white-parallel.

Corollary 4.8. Let $\Delta^{n} \subset \mathbb{R}^{n+1}$ be the standard simplex. Let $P_{1}, P_{2}, \ldots \subset E$ be a (possibly infinite) set of (n-1)-dimensional affine planes and let $Q_{i}=P_{i} \cap \Delta^{n}$ for $i=1,2, \ldots$. Assume that we have an associated canonical coloring.

Let $P_{i}$ be of type $\left\{0 a_{1}^{i} \ldots a_{c(i)}^{i}\right\}$, for $i=1,2, \ldots$ Then either

$-c(1) \in\{0, n-1\}$, or

- whenever, for some $i \in\{2,3, \ldots\}, P_{1}$ and $P_{i}$ bound a white component of $\Delta^{n}-\cup_{j} Q_{j}$, then they must have a white-parallel arc.

Proof. Assume that $c(1) \notin\{0, n-1\}$. The white component $W$ bounded by $P_{1}$ is bounded by a finite number of planes; thus we can apply Corollary 4.7, and conclude that $P_{1}$ has a white-parallel arc with each other plane adjacent to $W$.

Definition 4.9. Let $P \subset E$ be an (n-1)-dimensional affine plane and $T$ a triangulation of the polytope $Q:=P \cap \Delta^{n}$. We say that $T$ is minimal if all vertices of $T$ are vertices of $Q$. We say that an edge of some simplex in $T$ is an exterior edge if it is an edge of $Q$.

Observation 4.10. Let $P \subset E$ be an (n-1)-dimensional affine plane and $T$ a triangulation of the polytope $Q:=P \cap \Delta^{n}$. If $T$ is minimal, each edge of $Q$ is an (exterior) edge of (exactly one) simplex in $T$.

Proof. By minimality, the triangulation does not introduce new vertices. Thus every edge of $Q$ is an edge of some simplex.

Observation 4.11. Let $P \subset E$ be an (n-1)-dimensional affine plane with $Q:=$ $P \cap \Delta^{n} \neq \varnothing$. Assume that $P$ is of type $\left\{0 a_{1} \ldots a_{k}\right\}$. Then either

(a) Each vertex of $Q$ arises as the intersection of $P$ with an edge e of $\Delta^{n}$. The vertices of e are $v_{i}$ and $v_{j}$ with $i \in\left\{0, a_{1}, \ldots, a_{k}\right\}$ and $j \notin\left\{0, a_{1}, \ldots, a_{k}\right\}$. (We will denote such a vertex by $\left(v_{i} v_{j}\right)$.)

(b) Two vertices $\left(v_{i_{1}} v_{j_{1}}\right)$ and $\left(v_{i_{2}} v_{j_{2}}\right)$ of $Q$ are connected by an edge of $Q$ (i.e., an exterior edge of any triangulation) if either $i_{1}=i_{2}$ or $j_{1}=j_{2}$.

Proof. (a) holds because $e$ has to connect vertices in distinct components of $\Delta^{n}-Q$. Statement (b) holds because the edge of $Q$ has to belong to some 2-dimensional subsimplex of $\Delta^{n}$, with vertices either $v_{i_{1}}, v_{j_{1}}, v_{j_{2}}$ or $v_{i_{1}}, v_{i_{2}}, v_{j_{1}}$. 
Remark. If, for an affine hyperplane $P \subset E, Q=P \cap \Delta^{n}$ consists of exactly one vertex, then we will consider the minimal triangulation of $Q$ to consist of one (degenerate) $(n-1)$-simplex. This convention helps to avoid needless case distinctions.

Lemma 4.12. Let $\left\{P_{i} \subset E: i \in I\right\}$ be a set of (n-1)-dimensional affine planes and let $Q_{i}:=P_{i} \cap \Delta^{n}$ for $i \in I$. Assume that $Q_{i} \cap Q_{j}=\varnothing$ for all $i \neq j$ and that we have an associated canonical coloring. Assume that we have fixed, for each $i \in I$, a minimal triangulation $Q_{i}=\bigcup_{a} \tau_{i a}$ of $Q_{i}$.

If $P_{1}$ is of type $\left\{0 a_{1}^{1} \ldots a_{c(1)}^{1}\right\}$ with $1 \leq c(1) \leq n-2$, then for each simplex $\tau_{1 a} \subset$ $Q_{1}$ there exists some $j \in I$ and some simplex $\tau_{j b} \subset Q_{j}$ (of the fixed triangulation of $Q_{j}$ ) such that $\tau_{i a}$ and $\tau_{j b}$ have a white-parallel arc.

Proof. Let $w_{1}, \ldots, w_{n}$ be the $n$ vertices of the $(n-1)$-simplex $\tau_{1 k}$. By Observation 4.11(a), each $w_{l}$ arises as intersection of $Q_{1}$ with some edge $\left(v_{r_{l}} v_{s_{l}}\right)$ of $\Delta^{n}$, and the vertices $v_{r_{l}}, v_{s_{l}}$ satisfy $r_{l} \in\left\{0, a_{1}^{1}, \ldots, a_{c(1)}^{1}\right\}$ and $s_{l} \notin\left\{0, a_{1}^{1}, \ldots, a_{c(1)}^{1}\right\}$.

For the canonical coloring, there must be a white component $W$ bounded by $P_{1}$. We distinguish the cases whether $W$ and $v_{0}$ belong to the same connected component of $\Delta^{n}-Q_{1}$ or not.

Case 1: $W$ and $v_{0}$ belong to the same connected component of $\Delta^{n}-Q_{1}$.

Since $c(1) \leq n-2$, there exist at most $n-1$ possible values for $r_{l}$. Hence there exists $l \neq m \in\{1, \ldots, n\}$ such that $v_{r_{l}}=v_{r_{m}}$.

Let $e$ be the edge of $\tau_{1 k} \subset Q_{1}$ connecting $w_{l}$ and $w_{m}$. By Observation 4.11(b), $e$ is an exterior edge. Consider the 2-dimensional subsimplex $\tau^{2} \subset \Delta^{n}$ with vertices $v_{r_{l}}, v_{s_{l}}, v_{s_{m}}$. We conclude that $P_{1}$ intersects $\tau^{2}$ in $e$, i.e., in an arc separating $v_{r_{l}}$ from the other two vertices of $\tau^{2}$.

Note that $r_{l} \in\left\{0, a_{1}^{1}, \ldots, a_{c(1)}^{1}\right\}$; hence $v_{r_{l}}$ belongs to the same component of $\Delta^{n}-Q_{1}$ as $v_{0}$. In particular, $v_{r_{l}}$ belongs to the same component of $\Delta^{n}-Q_{1}$ as $W$. On the other hand, since the coloring is canonical, all vertices are colored black, and $v_{r_{l}}$ cannot belong to the white component $W$. Thus there must be some plane $P_{j}$ such that $Q_{j}$ bounds $W$ and separates $v_{r_{l}}$ from $Q_{1}$. (The possibility that $P_{j} \cap \Delta^{n}=\left\{v_{r_{l}}\right\}$ is allowed.) In particular, some (possibly degenerate) exterior edge $f$ of $Q_{j}$ separates $v_{r_{l}}$ from $v_{s_{l}}, v_{s_{m}}$. Thus $e$ and $f$ are white-parallel arcs. By Observation 4.10, $f$ is an edge of some $\tau_{j l}$.

Case 2: $W$ and $v_{0}$ don't belong to the same connected component of $\Delta^{n}-Q_{1}$. Since $n-c(1) \leq n-1$, there exist some $l \neq m \in\{1, \ldots, n\}$ such that $v_{s_{l}}=v_{s_{m}}$. Let $e$ be the edge of $\tau_{1 k} \subset Q_{1}$ connecting $w_{l}$ and $w_{m} . e$ is an exterior edge by Observation 4.11(b). Consider the 2-dimensional subsimplex $\tau^{2} \subset \Delta^{n}$ with vertices $v_{r_{l}}, v_{r_{m}}, v_{s_{l}}$. $P_{1}$ intersects $\tau^{2}$ in $e$, i.e., in an arc separating $v_{s_{l}}$ from the other two vertices of $\tau^{2}$. 
We have $s_{l} \notin\left\{0, a_{1}^{1}, \ldots, a_{c(1)}^{1}\right\}$; hence $v_{s_{l}}$ does not belong to the same component of $\Delta^{n}-Q_{1}$ as $v_{0}$. This implies that $v_{s_{l}}$ belongs to the same component of $\Delta^{n}-Q_{1}$ as $W$. On the other hand, since the coloring is canonical, $v_{s_{l}}$ cannot belong to the white component $W$ and there must be some plane $P_{j}$ such that $Q_{j}$ bounds $W$ and separates $v_{s_{l}}$ from $Q_{1}$. In particular, some exterior edge $f$ of $Q_{j}$ separates $v_{s_{l}}$ from $v_{r_{l}}, v_{r_{m}}$. Thus $e$ and $f$ are white-parallel arcs. By Observation 4.10, $f$ is an edge of some $\tau_{j l}$.

Lemma 4.13. Let $\left\{P_{i}: i \in I\right\}$ be a set of (n-1)-dimensional affine planes with $Q_{i}:=P_{i} \cap \Delta^{n} \neq \varnothing$ for $i \in I$. Let $P_{i}$ be of type $\left\{0 a_{1}^{(i)} \ldots a_{k_{i}}^{(i)}\right\}$ for $i \in I$. Assume that $Q_{i} \cap Q_{j}=\varnothing$ for $i \neq j \in I$, and that we have an associated canonical coloring. Assume that for each $Q_{i}$ one has fixed a minimal triangulation $Q_{i}=\cup_{k=1}^{t(i)} \tau_{i k}$.

For each $i \in I$, let

$D_{i}=\sharp\left\{\tau_{i k} \subset Q_{i}\right.$ : there is no $\tau_{j l} \subset Q_{j}$ such that $\tau_{i k}, \tau_{j l}$ have a white-parallel arc $\}$.

Then

$$
\sum_{i \in I} D_{i}=0 \quad \text { or } \quad \sum_{i \in I} D_{i}=n+1
$$

Proof. First we remark that the number of planes may be infinite, but we may of course remove pairs of planes $P_{i}, P_{j}$ whenever they are of the same type and bound the same white component. This removal of $P_{i}, P_{j}$ and the common white component does not affect $\sum_{i \in I} D_{i}$. Since there are only finitely many different types of planes, we may without loss assume that we start with a finite number $P_{1}, \ldots, P_{m}$ of planes. (It may happen that after this removal no planes and no white components remain. In this case $\sum_{i \in I} D_{i \in I}=0$.) So we assume now that we have a finite number of planes $P_{1}, \ldots, P_{m}$, and no two planes of the same type bound a white region.

The first case to consider is that all planes are of type $\left\{0 a_{1} \ldots a_{k}\right\}$ with $k=0$ or $k=n-1$. Since all vertices are colored black, this means that $m=n+1$ and (upon renumbering) $P_{1}$ is of type $\{0\}, P_{n+1}$ is of type $\{01 \ldots n-1\}$, and $P_{i}$ is of type $\{01 \ldots \widehat{i-1} \ldots n\}$ for $i=2, \ldots, n$. Hence $D_{1}=\ldots=D_{n+1}=1$ and $\sum_{i=1}^{n+1} D_{i}=n+1$.

Now we assume that there exists $P_{i}$, say $P_{1}$, of type $\left\{0 a_{1}^{(1)} \ldots a_{k_{1}}^{(1)}\right\}$ with $1 \leq$ $c(1) \leq n-2$. Let $W$ be the white component bounded by $P_{1}$ and, without loss of generality, let $P_{2}, \ldots, P_{l}$ be the other planes bounding $W$. Then Lemma 4.12 says that each simplex in the chosen triangulation of $Q_{1}$ has a parallel arc with some simplex in the chosen triangulation of each of $Q_{2}, \ldots, Q_{l}$. In particular, $D_{1}=0$. For $j \in\{2, \ldots, l\}$, if $1 \leq c(j) \leq n-2$, the same argument shows that $D_{j}=0$. If $j \in\{2, \ldots, l\}$ and $c(j)=0$ or $c(j)=n-1$, then $Q_{j}$ consists of only one simplex. By Corollary 4.8, this simplex has a parallel arc with (some exterior edge of) $Q_{1}$ and 
thus (by Observation 4.10) with some simplex of the chosen triangulation of $Q_{1}$. This shows that $D_{j}=0$ also in this case. Altogether we conclude that $\sum_{j=1}^{l} D_{j}=0$ and thus $\sum_{i=1}^{m} D_{i}=\sum_{i=l+1}^{m} D_{i}$. Hence we can remove ${ }^{4}$ the white component $W$ and its bounding planes $P_{1}, \ldots, P_{l}$ to obtain a smaller number of planes and a new canonical coloring without changing $\sum_{i=1}^{m} D_{i}$. Since we start with finitely many planes, we can repeat this reduction finitely many times and will end up either with an empty set of planes or with a set of planes of type $\left\{0 a_{1} \ldots a_{k}\right\}$, with $k=0$ or $k=n-1$. Thus either $\sum_{i=1}^{m} D_{i}=0$ or $\sum_{i=1}^{m} D_{i}=n+1$.

We have thus proved that, in the presence of a canonical coloring, the number of $(n-1)$-simplices without white-parallel arcs in a minimal triangulation of the $Q_{i}$ is 0 or $n+1$. We remark that in the proof of Theorem 1.1 we will actually count only those triangles that have neither a white-parallel arc nor a degenerate arc. Thus, in general, we may remain with even less than $n+1(n-1)$-simplices.

\section{A straightening procedure}

In this section we will always work with the following set of assumptions.

Assumption I. $Q$ is an aspherical n-dimensional manifold with aspherical boundary $\partial Q$. We have $(n-1)$-dimensional submanifolds $\partial_{0} Q, \partial_{1} Q \subset \partial Q$ such that $\partial Q=\partial_{0} Q \cup \partial_{1} Q, \partial \partial_{0} Q=\partial \partial_{1} Q$, and $\partial_{1} Q \neq \varnothing$ is aspherical.

The example that one should have in mind is a nonpositively curved manifold $Q$ with totally geodesic boundary $\partial_{1} Q$ and cusps corresponding to $\partial_{0} Q$.

In the case of nonpositively curved manifolds with totally geodesic boundary, there is a well-known straightening procedure that homotopes each relative cycle into a straight relative cycle. It is explained for closed hyperbolic manifolds in [Benedetti and Petronio 1992, Lemma C.4.3].

However, we will need a more subtle straightening procedure, which considers relative cycles with a certain 0-1 labeling of their edges and straightens the 1labeled edges into certain distinguished 1-simplices. This straightening procedure will be explained in Section 5C. Before that, we explain a construction which will "morally" (although not literally) reduce the proof of Theorem 1.1 to the case that $\partial_{0} Q \cap C$ is path-connected, for each path component $C$ of $\partial Q$.

\section{A. Making $\partial_{0} Q \cap C$ connected.}

Construction 5.1. Let Assumption I be satisfied. There exists a continuous map of triples $q:\left(Q, \partial Q, \partial_{1} Q\right) \rightarrow\left(Q, \partial Q, \partial_{1} Q\right)$ that is (as a map of triples) homotopic to the identity and such that, for each path component $C$ of $\partial Q$, the image $A:=$ $q\left(\partial_{0} Q \cap C\right)$ is path-connected.

\footnotetext{
${ }^{4}$ To remove a white component means that this component together with the neighboring black components will form one new black component.
} 
Moreover, for each path component $F$ of $\partial_{1} Q$, the path components of $\partial F \subset$ $\partial_{0} Q \cap \partial_{1} Q$ can be numbered by $E_{0}^{F}, \ldots, E_{s}^{F}$ and one can choose points $x_{E_{i}^{F}} \in E_{i}^{F}$ such that $q\left(x_{E_{i}^{F}}\right) \equiv x_{E_{0}^{F}}$ for $i=0, \ldots, s$.

Proof. For each path component $F$ of $\partial_{1} Q$, number the path components of $\partial F \subset$ $\partial_{0} Q \cap \partial_{1} Q$ by $E_{0}^{F}, \ldots, E_{s}^{F}$, where $s$ depends on $F$. Choose one point $x_{E}^{F} \in E$ for each path component $E \subset F$ of $\partial_{0} Q \cap \partial_{1} Q$. Whenever $E_{0}, E_{i}$ is a pair of path components of $\partial_{0} Q \cap \partial_{1} Q$ adjacent to the same path component $F$ of $\partial_{1} Q$, choose a 1-dimensional submanifold $l_{E_{0}^{F} E_{i}^{F}} \subset \partial_{1} Q$ with

$$
\partial l_{E_{0}^{F} E_{i}^{F}}=\left\{x_{E_{0}^{F}}\right\} \cup\left\{x_{E_{i}^{F}}\right\} .
$$

The $l_{E_{0}^{F} E_{i}^{F}}$ may be chosen succesively in such a way that they are disjoint from each other (apart from the common vertex $x_{E_{0}^{F}}$ ) and disjoint from $\partial_{0} Q$ (apart from the vertices $x_{E_{0}^{F}}$ and $x_{E_{i}^{F}}$.

For each pair $\left\{E_{0}^{F}, E_{i}^{F}\right\}$ let $h: l_{E_{0}^{F} E_{i}^{F}} \rightarrow\left\{x_{E_{0}^{F}}\right\}$ be the constant map from $l_{E_{0}^{F} E_{i}^{F}}$ to $x_{E_{0}^{F}}$. For each path component $F$ of $\partial_{1} Q$, the union

$$
\bigcup_{i=1}^{s} l_{E_{0}^{F} E_{i}^{F}}
$$

is an embedded wedge of arcs in $\partial_{1} Q$; hence it is contractible. In particular, $h$ is homotopic to the identity. By the homotopy extension property there exists $g: F \rightarrow F$ with

$$
\left.g\right|_{L_{0}^{F} E_{i}^{F}}=h \equiv x_{E_{0}}
$$

for all $l_{E_{0}^{F} E_{i}^{F}}$, and $g \sim$ id by a homotopy extending the homotopy between $h$ and id.

Thus we defined $g$ on each path component $F$ of $\partial_{1} Q$ with $F \cap \partial_{0} Q \neq \varnothing$. On path components $F$ of $\partial_{1} Q$ with $F \cap \partial_{0} Q=\varnothing$ we define $g=$ id. Hence we have defined $g$ on all of $\partial_{1} Q$.

On path components $C$ of $\partial_{0} Q$ with $C \cap \partial_{1} Q=\varnothing$, we define $f=$ id. Again by the homotopy extension property there exists $f: \partial Q \rightarrow \partial Q$ with $\left.f\right|_{\partial_{1} Q}=g$, $\left.f\right|_{C}=$ id for path components $C$ of $\partial_{0} Q$ with $C \cap \partial_{1} Q=\varnothing$, and $f \sim$ id by a homotopy extending the homotopy of $g$. (Of course, $f$ does not preserve the path components of $\partial_{0} Q$ that intersect $\partial_{1} Q$.)

Once again by the homotopy extension property there exists $q: Q \rightarrow Q$ with $q \sim$ id such that $q$ extends $f$ and the homotopy between $q$ and id extends the homotopy between $f$ and id.

Due to the stepwise construction, $q$ is a map of triples, homotopic to the identity by a homotopy of triples. Moreover, $A:=q\left(\partial_{0} Q \cap C\right)$ is path-connected for each component $C$ of $\partial Q$. Indeed, any two points in $\partial_{0} Q \cap C$ can be connected by a sequence of paths which either have image in $\partial_{0} Q$ or belong to $\bigcup_{i=1}^{s} l_{E_{0}^{F} E_{i}^{F}}$ for 
some path component $F$ of $\partial_{1} Q \cap C$. The image of these paths under $q$, in both cases, is in $A$.

Remark. The map $q$ induces a simplicial map $q: K(Q) \rightarrow K(Q)$ and a homomorphism $q_{*}: \Pi\left(K\left(\partial_{0} Q\right)\right) \rightarrow \Pi(K(A))$ defined by

$$
q_{*}\left(\left\{\gamma_{1}, \ldots, \gamma_{n}\right\}\right):=\left\{q\left(\gamma_{1}\right), \ldots, q\left(\gamma_{n}\right)\right\},
$$

such that $q_{*}(g) q(\sigma)=q(g \sigma)$ for each $\sigma \in K(Q)$ and $g \in \Pi\left(K\left(\partial_{0} Q\right)\right)$.

Proof. Continuous maps $q: Q \rightarrow Q$ induce simplicial maps $q: K(Q) \rightarrow K(Q)$. (The simplicial map agrees with $q$ on the 0 -skeleton, and it maps each 1-simplex $e \in K_{1}(Q)$ to the unique 1 -simplex of $K_{1}(Q)$ that is in the homotopy class rel $\{0,1\}$ of $q(e)$.)

Let $e \in K_{1}(Q)$. By construction, $\left\{\gamma_{1}, \ldots, \gamma_{n}\right\} e=[\alpha * e * \bar{\beta}]$ for some $\alpha, \beta \in$ $\left\{\gamma_{1}, \ldots, \gamma_{n}\right\} \cup\left\{c_{e(0)}, c_{e(1)}\right\}$. Thus

$$
\left\{q\left(\gamma_{1}\right), \ldots, q\left(\gamma_{n}\right)\right\} q(e)=[q(\alpha) * q(e) * q(\bar{\beta})]=q\left(\left\{\gamma_{1}, \ldots, \gamma_{n}\right\} e\right) .
$$

This implies the claim for the 1-skeleton, and thus, by the asphericity of $K(Q)$, for all $\sigma \in K(Q)$.

5B. Definition of $\boldsymbol{K}^{\text {str }}(\boldsymbol{Q})$. Let $Q, \partial Q, \partial_{1} Q, \partial_{0} Q$ satisfy Assumption I.

Recall that we have defined in Section 3B an aspherical multicomplex $K(Q) \subset$ $S_{*}(Q)$ with the property that (for aspherical $Q$ ) each singular simplex in $Q$, with boundary in $K(Q)$ and pairwise distinct vertices, is homotopic rel boundary to a unique simplex in $K(Q)$.

The aim of this subsection is to describe a selection procedure yielding a subset $K_{*}^{\text {str }}(Q) \subset S_{*}(Q)$. The final purpose of the straightening procedure will be to produce a large number of (weakly) degenerate simplices, in the sense of the following definition.

Definition 5.2. Let $Q$ be an compact manifold with boundary $\partial Q$. We say that a simplex in $S_{*}(Q)$ is degenerate if one of its edges is a constant loop. We say that it is weakly degenerate if it is degenerate or its image is contained in $\partial Q$.

Notational remark. For subsets $K_{*}^{\mathrm{str}}(Q) \subset S_{*}(Q)$ we define

$$
\begin{aligned}
K_{*}^{\mathrm{str}}\left(\partial_{0} Q\right) & :=K_{*}^{\mathrm{str}}(Q) \cap S_{*}\left(\partial_{0} Q\right), \\
K_{*}^{\mathrm{str}}\left(\partial_{1} Q\right) & :=K_{*}^{\mathrm{str}}(Q) \cap S_{*}\left(\partial_{1} Q\right), \\
K_{*}^{\mathrm{str}}\left(\partial_{0} Q Q\right) & :=K_{*}^{\mathrm{str}}(Q) \cap S_{*}\left(\partial_{0} Q\right) .
\end{aligned}
$$

Lemma 5.3. Let $Q, \partial Q, \partial_{1} Q, \partial_{0} Q$ satisfy Assumption I. Let $K(Q) \subset S_{*}(Q)$ be as defined in Section 3B. Let $q: Q \rightarrow Q$ and $\left\{x_{E_{i}^{F}} \in \partial_{0} Q \cap \partial_{1} Q: 0 \leq i \leq s\right\}$ be given by Construction 5.1.

Then there exists a subset $K_{*}^{\text {str }}(Q) \subset S_{*}(Q)$, closed under face maps, such that: 
(i) If $C$ is a path component of $\partial_{0} Q$ with $C \cap \partial_{1} Q=\varnothing$, then $K_{0}^{\mathrm{str}}(Q)$ contains each point in $C$.

(ii) For a path component $F$ of $\partial_{1} Q$ with $F \cap \partial_{0} Q=\varnothing$, there is exactly one point $x_{F} \in K_{0}^{\mathrm{str}}(Q) \cap F$, while

for a path component $F$ of $\partial_{1} Q$ with $F \cap \partial_{0} Q \neq \varnothing$, we have $K_{0}^{\text {str }}(Q) \cap F=$ $\left\{x_{E_{0}^{F}}, \ldots, x_{E_{s}^{F}}\right\}$.

(iii) $K_{0}^{\mathrm{str}}(Q)=K_{0}^{\mathrm{str}}(\partial Q)$.

(iv) $K_{1}^{\mathrm{str}}(Q)$ consists of

- all 1-simplices $e \in K(Q)$ with $\partial e \in K_{0}^{\mathrm{str}}(Q)$,

- exactly one 1-simplex for each nontrivial homotopy class (rel boundary) of loops e with $\partial_{0} e=\partial_{1} e \in K_{0}^{\mathrm{str}}(Q)$, and

- the constant loop for the homotopy class of the constant loop at $x$, if $x \in K_{0}^{\mathrm{str}}(Q)$.

(v) For $n \geq 2$, if $\sigma \in S_{n}(Q)$ is an n-simplex with $\partial \sigma \in K_{n-1}^{\mathrm{str}}(Q)$, then $\sigma$ is homotopic rel boundary to a unique $\tau \in K_{n}^{\mathrm{str}}(Q)$.

(vi) If $\sigma \in K_{n}^{\mathrm{str}}(Q)$ is homotopic rel boundary to some $\tau \in K_{n}(Q)$, then $\sigma=\tau$.

(vii) If $\sigma \in K_{n}^{\mathrm{str}}(Q)$ is homotopic rel boundary to a simplex $\tau \in S_{n}\left(\partial_{1} Q\right)$, then $\sigma \in K_{n}^{\mathrm{str}}\left(\partial_{1} Q\right)$; if $\sigma \in K_{1}^{\mathrm{str}}(Q)$ is homotopic rel boundary to a simplex $\tau \in$ $S_{1}\left(\partial_{0} Q\right)$, then $\sigma \in K_{1}^{\mathrm{str}}\left(\partial_{0} Q\right)$.

(viii) $K_{*}^{\mathrm{str}}(Q)$ is aspherical, i.e., if $\sigma, \tau \in K_{*}^{\mathrm{str}}(Q)$ have the same 1-skeleton, then $\sigma=\tau$.

Proof. $K_{*}^{\text {str }}(Q)$ is defined by induction on the dimension of simplices as follows. Definition of $K_{0}^{\text {str }}(Q)$ : Choose $K_{0}^{\text {str }}(Q)$ such that conditions (i)-(iii) are satisfied. Note that we have chosen a nonempty set of 0 -simplices since we are assuming $\partial_{1} Q \neq \varnothing$.

Definition of $K_{1}^{\text {str }}(Q)$ : For an ordered pair $(x, y) \in K_{0}^{\text {str }}(Q) \times K_{0}^{\text {str }}(Q)$ with $x \neq y$, there exists unique simplex in $K_{1}(Q)$ in each homotopy class (rel boundary) of arcs $e$ from $x$ to $y$. Choose these 1-simplices so they belong to $K_{1}^{\text {str }}(Q)$. (Uniqueness implies that (vi) is true for $n=1$.) For pairs $(x, x) \in K_{0}^{\mathrm{str}}(Q) \times K_{0}^{\mathrm{str}}(Q)$, choose one simplex in each homotopy class (rel boundary) of loops $e$ from $x$ to itself. For the homotopy class of the constant loop, choose the constant loop.

Choose the 1-simplices in $\partial_{0} Q$ and/or $\partial_{1} Q$ whenever this is possible. (If a 1simplex is homotopic into both $\partial_{0} Q$ and $\partial_{1} Q$, then it is necessarily homotopic into $\partial_{0} Q \cap \partial_{1} Q$. Indeed, a disk realizing a homotopy between 1 -simplices in $\partial_{0} Q$ and $\partial_{1} Q$ can be made transversal to $\partial_{0} Q \cap \partial_{1} Q$ and then intersects $\partial_{0} Q \cap \partial_{1} Q$ in an arc or loop.) Hence (vii) is satisfied for $n=1$. 
Definition of $K_{n}^{\text {str }}(Q)$ for $n \geq 2$, assuming that $K_{n-1}^{\text {str }}(Q)$ is defined: For an $(n+1)$ tuple $\kappa_{0}, \ldots, \kappa_{n}$ of $(n-1)$-simplices in $K_{n-1}^{\text {str }}(Q)$, satisfying $\partial_{i} \kappa_{j}=\partial_{j-1} \kappa_{i}$ for all $i, j$, there are two possibilities:

- If no edge of any $\kappa_{i}$ is a loop, then, by the asphericity of $Q$, there is a unique $n$-simplex $\sigma \in K_{n}(Q)$ with $\partial_{i} \sigma=\kappa_{i}$ for $i=0, \ldots, n$. In this case set $\kappa:=\sigma$. Uniqueness implies that (vi) is satisfied for $n$. (By the construction in Section 3B, we have $\kappa \in K_{n}\left(\partial_{1} Q\right)$ if $\kappa$ is homotopic rel boundary into $\partial_{1} Q$.)

- Otherwise, choose an $n$-simplex $\kappa \in S_{n}(Q)$ with $\partial_{i} \kappa=\kappa_{i}$ for $i=0, \ldots, n$. Since $Q$ is aspherical, $\kappa$ exists and is unique up to homotopy rel boundary. Choose the simplices in $\partial_{1} Q$ whenever this is possible.

By construction, $K_{*}^{\text {str }}(Q)$ is closed under face maps and satisfies the conditions (i)-(vii). Condition (viii) follows by induction on the dimension of subsimplices of $\sigma$ and $\tau$ from condition (v).

The simplices in $K_{*}^{\mathrm{str}}(Q)$ will be called straight simplices.

We remark that $K_{*}^{\mathrm{str}}(Q)$ is not a multicomplex because simplices in $K_{*}^{\mathrm{str}}(Q)$ need not have pairwise distinct vertices. (Note also that simplices in $K(Q)$ belong to $K^{\mathrm{str}}(Q)$ if and only if all their vertices belong to $K_{0}^{\text {str }}(Q)$, by construction.)

Observation 5.4. Let $Q, \partial Q, \partial_{1} Q, \partial_{0} Q$ satisfy Assumption I. Let $K_{*}^{\operatorname{str}}(Q) \subset S_{*}(Q)$ satisfy conditions (i)-(viii) from Lemma 5.3. Then $q: Q \rightarrow Q$ induces a simplicial map $q: K^{\operatorname{str}}(Q) \rightarrow K^{\text {str }}(Q)$, compatible with the simplicial map $q: K(Q) \rightarrow K(Q)$ from Section $5 A$.

Proof. By construction, $q$ maps $K_{0}^{\text {str }}(Q)$ to itself. Indeed:

- If $C$ is a path component of $\partial_{0} Q$ with $C \cap \partial_{1} Q=\varnothing$, then $q(v)=v$ for each $v \in C$.

- If $F$ is a path component $F$ of $\partial_{1} Q$ with $F \cap \partial_{0} Q=\varnothing$, then $q(v)=v$ for each $v \in F$ (in particular for the unique $v \in F \cap K_{0}^{\text {str }}(Q)$ ).

- If $F$ is a path component of $\partial_{1} Q$ with $F \cap \partial_{0} Q \neq \varnothing$, then we have $K_{0}^{\text {str }}(Q) \cap$ $F=\left\{x_{E_{0}^{F}}, \ldots, x_{E_{s}^{F}}\right\}$, and $q\left(x_{E_{i}^{F}}\right)=x_{E_{0}^{F}}$ for $i=0, \ldots, s$ by Construction 5.1.

Hence $q$ induces a simplicial map on $K^{\text {str }}(Q)$. (The simplicial map agrees with $q$ on the 0 -skeleton, and it maps each 1-simplex $e \in K_{1}^{\text {str }}(Q)$ to the unique 1simplex of $K_{1}^{\text {str }}(Q)$ that is in the homotopy class rel $\{0,1\}$ of $q(e)$. Since $K^{\text {str }}(Q)$ is aspherical, this determines the simplicial map $q$ uniquely.)

\section{C. Definition of the straightening.}

Definition 5.5. Let $\left(Q, \partial_{1} Q\right)$ be a pair of topological spaces and let $z=\sum_{i \in I} a_{i} \tau_{i} \in$ $C_{n}^{\text {inf }}(Q)$ be a (possibly infinite) singular chain. 
(a) A set of cancellations of $z$ is a symmetric set $\mathscr{C} \subset S_{n-1}(Q) \times S_{n-1}(Q)$ with $\left(\eta_{1}, \eta_{2}\right) \in \mathscr{C} \Rightarrow \eta_{1}=\eta_{2}$ and $\eta_{1}=\partial_{k} \tau_{i_{1}}, \eta_{2}=\partial_{l} \tau_{i_{2}}$ for some $i_{1}, i_{2} \in I$ and $k, l \in\{0, \ldots, n\}$.

(b) Let $z=\sum_{i \in I} a_{i} \tau_{i} \in C_{n}^{\inf }(Q)$. If $\mathscr{C}$ is a set of cancellations for $z$, the associated simplicial set $\Upsilon_{z, \mathscr{C}}$ is the simplicial set generated ${ }^{5}$ by $\left\{\Delta_{i}: i \in I\right\}$, subject to the identifications $\partial_{k} \Delta_{i_{1}}=\partial_{l} \Delta_{i_{2}}$ if and only if $\left(\partial_{k} \tau_{i_{1}}, \partial_{l} \tau_{i_{2}}\right) \in \mathscr{C}$.

(c) Let $z=\sum_{i \in I} a_{i} \tau_{i} \in C_{n}^{\text {inf }}(Q)$. Choose a minimal presentation for $\partial z$ (meaning that no further cancellation is possible). Define

$$
\begin{array}{r}
J=J_{\partial z}:=\left\{(i, a) \in I \times\{0, \ldots, n\}: \partial_{a} \tau_{i}\right. \text { occurs with a nonzero coefficient } \\
\text { in the chosen presentation of } \partial z\} .
\end{array}
$$

Let $\mathscr{C}$ be a set of cancellations for $z$. Then the simplicial set $\partial \Upsilon_{z, \mathscr{C}}$ is defined as the set consisting of $|J|(n-1)$-simplices $\Delta_{i, a},(i, a) \in J$, together with all their iterated faces and degenerations, subject to the identifications $\partial_{a} \partial_{a_{1}} \tau_{i_{1}}=$ $\partial_{a} \partial_{a_{2}} \tau_{i_{2}}$ for all $a=0, \ldots, n-1$, whenever $\left(\partial_{a_{1}} \tau_{i_{1}}, \partial_{a_{2}} \tau_{i_{2}}\right) \in \mathscr{C}$ and $\left(i_{1}, a_{1}\right) \in J$.

(d) If $z=\sum_{i \in I} a_{i} \tau_{i} \in C_{n}^{\inf }(Q)$ is a relative cycle, then a set of cancellations $\mathscr{C}$ is called sufficient if the formal sum $\sum_{i \in I} \sum_{k=0}^{n}(-1)^{k} a_{i} \partial_{k} \tau_{i}$ can be reduced to a chain in $C_{n-1}^{\inf }(\partial Q)$ by substracting (possibly infinitely many) multiples of $\left(\partial_{a_{1}} \tau_{i_{1}}-\partial_{a_{2}} \tau_{i_{2}}\right)$ with $\left(\partial_{a_{1}} \tau_{i_{1}}, \partial_{a_{2}} \tau_{i_{2}}\right) \in \mathscr{C}$.

Observations 5.6. Let $\left(Q, \partial_{1} Q\right)$ be a pair of topological spaces.

(a) If $z=\sum_{i \in I} a_{i} \tau_{i} \in C_{n}^{\inf }(Q)$ is a singular chain, $\mathscr{C}$ is a set of cancellations, and $\Upsilon:=\Upsilon_{z, \mathscr{C}}$ is the associated simplicial set, the geometric realization $|\Upsilon|$ is obtained from $|I|$ copies of the standard $n$-simplex $\Delta_{i}, i \in I$, with identifications $\partial_{a_{1}} \Delta_{i_{1}}=\partial_{a_{2}} \Delta_{i_{2}}$ if and only if $\left(\partial_{a_{1}} \tau_{i_{1}}, \partial_{a_{2}} \tau_{i_{2}}\right) \in \mathscr{C}$. For a minimal presentation of $\partial z$ and $\partial \Upsilon:=\partial \Upsilon_{z, \mathscr{C}},|\partial \Upsilon|$ is the subspace of $|\Upsilon|$ containing all simplices $\partial_{a_{1}} \Delta_{i_{1}}$ with $\left(i_{1}, a_{1}\right) \in J$.

(b) There exists an associated continuous map $\tau$ : $|\Upsilon| \rightarrow Q$ with $\tau \mid \Delta_{i}=\tau_{i}$ (upon the identification $\left.\Delta_{i}=\Delta^{n}\right)$. If $z$ is a relative cycle, i.e., if $\partial z \in C_{n-1}^{\inf }\left(\partial_{1} Q\right)$, then $\tau$ maps $|\partial \Upsilon|$ to $\partial_{1} Q$.

(c) Let $z_{1}=\sum_{i \in I} a_{i} \tau_{i}, z_{2}=\sum_{i \in I} a_{i} \sigma_{i} \in C_{n}^{\text {inf }}\left(Q, \partial_{1} Q\right)$ be relative cycles and let $\mathscr{C}_{1}, \mathscr{C}_{2}$ be sufficient sets of cancellations of $z_{1}$ and $z_{2}$, respectively. Assume that $\left(\partial_{a_{1}} \tau_{i_{1}}, \partial_{a_{2}} \tau_{i_{2}}\right) \in \mathscr{C}_{1}$ if and only if $\left(\partial_{a_{1}} \sigma_{i_{1}}, \partial_{a_{2}} \sigma_{i_{2}}\right) \in \mathscr{C}_{2}$, and that there exist minimal presentations of $\partial z_{1}, \partial z_{2}$ such that $J_{z_{1}}=J_{z_{2}}$.

If the associated continuous maps $\tau, \sigma:|\Upsilon| \rightarrow Q$ are homotopic, for a homotopy mapping $|\partial \Upsilon|$ to $\partial Q$, then $\sum_{i \in I} a_{i} \tau_{i}$ and $\sum_{i \in I} a_{i} \sigma_{i} \in C_{*}^{\text {inf }}(Q, \partial Q)$ are relatively homologous.

${ }^{5}$ That is, the subset of $S_{*}^{\text {sing }}(Q)$ containing the $|I| n$-simplices $\Delta_{i}, i \in I$, together with all simplices obtained by iterated applications of face and degeneracy operators. See [May 1967, Example 1.5]. 
We emphasize that we do not assume that $\mathscr{C}$ is a complete list of cancellations, and the simplicial map $\tau_{*}: C_{*}^{\text {simp }}(\Upsilon) \rightarrow C_{*}^{\text {sing }}(Q)$ need not be injective.

After having set up the necessary notations, we will now define the actual straightening. We first mention that there is of course an analogue of the classical straightening of [Benedetti and Petronio 1992, Lemma C.4.3] in our setting.

Observation 5.7. Let $Q, \partial Q, \partial_{1} Q, \partial_{0} Q$ satisfy Assumption I. Suppose $K_{*}^{\mathrm{str}}(Q) \subset$ $S_{*}(Q)$ satisfy conditions (i)-(viii) from Lemma 5.3. Then there exists a "canonical straightening" map

$$
\operatorname{str}_{\text {can }}: C_{*}^{\text {simp,inf }}(K(Q)) \rightarrow C_{*}^{\text {simp, inf }}\left(K^{\mathrm{str}}(Q)\right),
$$

mapping $C_{*}^{\mathrm{simp}, \inf }\left(K\left(\partial_{1} Q\right)\right)$ to $C_{*}^{\mathrm{simp}, \inf }\left(K^{\mathrm{str}}\left(\partial_{1} Q\right)\right)$, with the following properties:

(i) $\operatorname{str}_{\mathrm{can}}$ is a chain map.

(ii) If $z=\sum_{i \in I} a_{i} \tau_{i} \in C_{*}^{\operatorname{simp}, \inf }(K(Q))$ and $\sum_{i \in I} a_{i} \sigma_{i}:=\sum_{i \in I} a_{i} \operatorname{str}_{\mathrm{can}}\left(\tau_{i}\right)$, then the maps $\tau, \sigma:|\Upsilon| \rightarrow Q$ (defined by Observation 5.6(b) after fixing a set of cancellations $\mathscr{C}$ and a minimal presentation of $\partial z$ ) are homotopic.

Moreover, if $z=\sum_{i \in I} a_{i} \tau_{i}$ is a relative cycle with $\partial z \in C_{*}^{\operatorname{simp}, \inf }\left(K\left(\partial_{1} Q\right)\right)$, the same is true for $\sum_{i \in I} a_{i} \sigma_{i}$, and

$$
\tau, \sigma:(|\Upsilon|,|\partial \Upsilon|) \rightarrow\left(Q, \partial_{1} Q\right)
$$

are homotopic as maps of pairs.

In particular, $\sum_{i \in I} a_{i} \operatorname{str}_{\mathrm{can}}\left(\tau_{i}\right)$ is relatively homologous to $\sum_{i \in I} a_{i} \tau_{i}$.

Proof. We define $\operatorname{str}_{\mathrm{can}}$, and the homotopy to the identity, by induction on the dimension of simplices. (During the construction we take care that $\operatorname{str}_{\mathrm{can}}$ and the homotopy preserve $K\left(\partial_{1} Q\right)$.)

0 -simplices. If $C$ is a path component of $\partial_{0} Q$ with $C \cap \partial_{1} Q=\varnothing$, we define $\overline{\operatorname{str}_{\text {can }}(v)=v}$ for each 0 -simplex $v$ in $C$. The homotopy $H(v)$ is for each $v$ given by the constant map.

If $C$ is a path component of $\partial_{0} Q$ with $C \cap \partial_{1} Q \neq \varnothing$, there is at least one path component $F$ of $\partial_{1} Q$ with $C \cap F \neq \varnothing$. By Construction 5.1 and condition (ii) from Lemma 5.3, for each such $F$, there is a straight 0 -simplex $x_{E_{i}^{F}} \in C \cap F$. Choose one such straight 0-simplex (among the $x_{E_{i}^{F}}$ ) for each path component $C$ of $\partial_{0} Q$, denote it $x_{C}$, and for each $v \in C$ we define $\operatorname{str}_{\text {can }}(v):=x_{C} \in K_{0}^{\text {str }}(Q) \cap C$ and we choose the homotopy $H(v)$ to belong to $C$.

If $v \in \partial_{1} Q$, then there is (at least) one straight 0 -simplex in the same path component $F$ of $\partial_{1} Q$, we choose $\operatorname{str}_{\text {can }}(v) \in F \cap K_{0}^{\text {str }}(Q)$ and there exists $H(v) \in K_{1}\left(\partial_{1} Q\right)$ with $\partial H(v)=v-\operatorname{str}_{\mathrm{can}}(v)$.

If $v \notin \partial Q$, then we define $\operatorname{str}_{\text {can }}(v)$ to be some straight 0 -simplex in $\partial Q$ and we fix arbitrarily some $H(v) \in K_{1}(Q)$ with $\partial H(v)=v-\operatorname{str}_{\text {can }}(v)$. 
1-simplices. For $e \in K_{1}(Q)$ we define

$$
\operatorname{str}_{\mathrm{can}}(e):=\left[\overline{H\left(\partial_{1} e\right)} * e * H\left(\partial_{0} e\right)\right],
$$

where, as always, $[\cdot]$ denotes the unique 1 -simplex in $K_{1}^{\mathrm{str}}(Q)$, that is homotopic rel boundary to the path in brackets.

The simplex $e$ is homotopic to $\operatorname{str}_{\text {can }}(e)$ by the canonical homotopy that is inverse to the homotopy moving $\overline{H\left(\partial_{1} e\right)}$ or $H\left(\partial_{0} e\right)$ into constant maps. In particular, the restriction of this homotopy to $\partial_{1} e, \partial_{0} e$ gives $H\left(\partial_{1} e\right), \overline{H\left(\partial_{0} e\right)}$. Thus, for different edges with common vertices, the homotopies are compatible. We thus have constructed a homotopy for the 1-skeleton $\Upsilon_{1}$.

We note that, for $v \in \partial_{1} Q$, the homotopy $H(v)$ is either constant or lies in $K_{1}\left(\partial_{1} Q\right)$, Thus if $\tau \in K_{1}\left(\partial_{1} Q\right)$ then $\operatorname{str}_{\text {can }}(\tau) \in K_{1}^{\text {str }}\left(\partial_{1} Q\right)$ and the homotopy between $\tau$ and $\operatorname{str}_{\text {can }}(\tau)$ takes place in $\partial_{1} Q$.

$n$-simplices. We assume inductively, that for some $n \geq 1$, we have defined $\operatorname{str}_{\text {can }}$ on $K_{* \leq n}(Q)$, mapping $K_{* \leq n}\left(\partial_{1} Q\right)$ to $K_{* \leq n}^{\text {str }}\left(\partial_{1} Q\right)$, and satisfying (i) and (ii).

Let $\tau \in K(Q)$ be an $(n+1)$-simplex. Then we have by (ii) a homotopy between $\partial \tau$ and $\operatorname{str}_{\operatorname{can}}(\partial \tau)$. By Observation 3.1 this homotopy extends to $\tau$. The resulting simplex $\tau^{\prime}$ satisfies $\partial \tau^{\prime} \in K_{n}^{\text {str }}(Q)$. Condition (v) from Lemma 5.3 means that $\tau^{\prime}$ is homotopic rel boundary to a unique $\operatorname{simplex} \operatorname{str}_{\text {can }}(\tau) \in K_{n+1}^{\mathrm{str}}(Q)$. This proves the inductive step.

If $\tau \in K\left(\partial_{1} Q\right)$, then we can inductively assume that the homotopy of $\partial \tau$ has im-

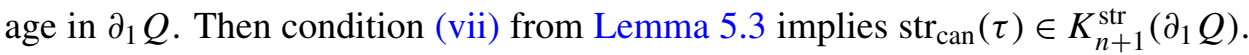
Moreover, since $\partial_{1} Q$ is aspherical, the homotopy of $\tau$ can be chosen to have image in $\partial_{1} Q$.

By construction, for any set of cancellations $\mathscr{C}$, the induced maps $\tau$ and $\sigma$ are homotopic. In particular, if we chose a sufficient set of cancellations in the sense of Definition 5.5(d), then Observation 5.6(c) implies that $\sum_{i=1}^{r} a_{i} \operatorname{str}_{\mathrm{can}}\left(\tau_{i}\right)$ is (relatively) homologous to $\sum_{i=1}^{r} a_{i} \tau_{i}$.

However, we want to define a more refined straightening, which will be defined only on relative cycles with some kind of additional information.

Before stating the definition of distinguished 1-simplices, we remark that there is a left and right action of the pseudogroup $\Gamma:=\Omega(\partial Q)$ (as defined in Section 3D) on $K_{1}^{\mathrm{str}}(Q):$ if $e \in K_{1}^{\mathrm{str}}(Q), \gamma_{1} \in \pi_{1}\left(\partial Q, \partial_{1} e\right), \gamma_{2} \in \pi_{1}\left(\partial Q, \partial_{0} e\right)$, then let $\gamma_{1} e \gamma_{2}$ be the unique straight 1-simplex homotopic rel $\{0,1\}$ to $\gamma_{1} * e * \gamma_{2}$. (The left action agrees with the action defined in Section 3D.) The cosets $\Gamma K_{1}^{\mathrm{str}}(Q) \Gamma$ in Definition 5.8 are with respect to this action.

For $x, y \in K_{0}^{\mathrm{str}}(Q)$ we will denote $K_{1, x y}^{\mathrm{str}}:=\left\{e \in K_{1}^{\mathrm{str}}(Q): \partial_{1} e=x, \partial_{0} e=y\right\}$.

Definition 5.8. Let $Q, \partial Q, \partial_{1} Q, \partial_{0} Q$ satisfy Assumption I. 
Let $q: Q \rightarrow Q$ and $\left\{x_{E_{i}^{F}} \in \partial_{0} Q \cap \partial_{1} Q\right\}$ be given by Construction 5.1. Let $K_{*}^{\text {str }}(Q) \subset S_{*}(Q)$ satisfy conditions (i)-(viii) from Lemma 5.3.

A set $D \subset K_{1}^{\text {str }}(Q)$ is called a set of distinguished 1-simplices if it satisfies the following conditions:

(ix) $\partial_{0} e, \partial_{1} e \in K_{0}^{\text {str }}(Q)$ for each $e \in D$.

(x) For each $(x, y) \in K_{0}^{\mathrm{str}}(Q) \times K_{0}^{\mathrm{str}}(Q)$, the set

$$
D_{x y}:=\left\{e \in D: \partial_{1} e=x, \partial_{0}=y\right\}
$$

contains exactly one element in each double coset $\Gamma f \Gamma \in \Gamma K_{1, x y}^{\mathrm{str}}(Q) \Gamma$, where $\Gamma=\Omega(\partial Q)$.

(xi) For all $x \in K_{0}^{\text {str }}(Q)$, the constant loop $c_{x}$ belongs to $D$.

(xii) If $e \in D$, then $\bar{e} \in D$, where the bar denotes orientation reversal.

(xiii) If $F, F^{\prime}$ are path components of $\partial_{1} Q$ and

$$
\left\{x_{E_{i}^{F}} \in \partial_{0} Q \cap F\right\}, \quad\left\{x_{E_{j}^{F^{\prime}}} \in \partial_{0} Q \cap F^{\prime}\right\}
$$

are given by Construction 5.1, then $q\left(D_{x_{E_{i}^{F}} x_{E_{j}^{F^{\prime}}}}\right)=D_{x_{E_{0}^{F}} x_{E_{0}^{F^{\prime}}}}$ for all $x_{E_{i}^{F}}, x_{E_{j}^{F^{\prime}}}$.

(xiv) If $x_{1}, x_{2} \in C_{1}$ and $y_{1}, y_{2} \in C_{2}$ for some path components $C_{1}, C_{2}$ of $\partial Q$, then for each $e_{1} \in D_{x_{1} y_{1}}$ there exists some $e_{2} \in D_{x_{2} y_{2}}$ with $q\left(e_{2}\right)=g q\left(e_{1}\right)$ for some $g \in H:=q_{*}\left(\Pi\left(K\left(\partial_{0} Q\right)\right)\right)$.

In connection with (xiii) we note that if $F \cap \partial_{0} Q=\varnothing$, there is only one straight 0 -simplex $x_{E_{0}^{F}}$ in $F$. Similarly, if $F^{\prime} \cap \partial_{0} Q=\varnothing$, there is only one straight 0 -simplex $x_{E_{0}^{F^{\prime}}}$ in $F^{\prime}$. In particular, if $F \cap \partial_{0} Q=\varnothing$ and $F^{\prime} \cap \partial_{0} Q=\varnothing$, condition (xiii) is empty.

Observation 5.9. Let the assumptions of Definition 5.8 be satisfied. Then a set $D$ of distinguished 1-simplices exists.

Proof. For each path component $C$ of $\partial Q$ we fix some $x_{C} \in j_{0}^{\text {str }}(C)$.

For each pair $\left\{C_{1}, C_{2}\right\}$ of path components, we select for membership in $D_{x_{C_{1}} x_{C_{2}}}$ one simplex $e$ with

$$
\partial_{1} e=x_{C_{1}}, \quad \partial_{0} e=x_{C_{2}}
$$

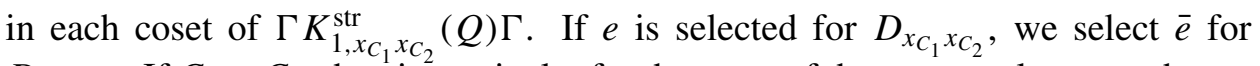
$D_{x_{C_{2}} x_{C_{1}}}$. If $C_{1}=C_{2}$, then in particular for the coset of the constant loop we choose the constant loop for $D_{x_{C_{1}} x_{C_{2}}}$.

For each path component $C$ of $\partial Q$ and each path component $F$ of $C \cap \partial_{1} Q$, we conclude that $q\left(x_{C}\right)$ and $q\left(x_{E_{0}^{F}}\right)$ belong to the path-connected set $q\left(\partial_{0} Q \cap C\right)$. 
Therefore we have a sequence of 1 -simplices $\alpha_{1}, \ldots, \alpha_{m} \in K_{1}\left(\partial_{0} Q\right)$ with images in distinct path components of $\partial_{0} Q \cap C$, such that

$$
\begin{aligned}
\partial_{1} q\left(\alpha_{1}\right) & =q\left(x_{C}\right), & \partial_{0} q\left(\alpha_{1}\right) & =\partial_{1} q\left(\alpha_{2}\right), \quad \ldots, \\
\partial_{0} q\left(\alpha_{m-1}\right) & =\partial_{1} q\left(\alpha_{m}\right), & \partial_{0} q\left(\alpha_{m}\right) & =q\left(x_{E_{0}^{F}}\right) .
\end{aligned}
$$

To prepare the definition of the $D_{x, y}$, we first describe, for each $x \in C \cap K_{0}^{\mathrm{str}}(Q)$, a sequence $\left\{\alpha_{1}, \ldots, \alpha_{k}\right\}$ of 1-simplices:

- If $C \cap \partial_{1} Q=\varnothing$, then $k=1$ and for each $x \in C$ we choose arbitrarily a 1-simplex $\alpha_{1}$ in $C$ with $\partial_{1} \alpha_{1}=x_{C}, \partial_{0} \alpha_{1}=x$.

- If $C \cap \partial_{0} Q=\varnothing$, then $C \cap K_{0}^{\mathrm{str}}(Q)=\left\{x_{C}\right\}$ by condition (ii) of Lemma 5.3, and we let $k=0$.

- If $C \cap \partial_{0} Q \cap \partial_{1} Q \neq \varnothing$, again by condition (ii) of Lemma 5.3 we have $x=x_{E_{i}^{F}}$ for some path component $F$ of $\partial_{1} Q$ and some $i$; thus we have the sequence $\alpha_{1}, \ldots, \alpha_{m}$ constructed above with $\partial_{1} q\left(\alpha_{1}\right)=q\left(x_{C}\right), \partial_{0} q\left(\alpha_{1}\right)=\partial_{1} q\left(\alpha_{2}\right), \ldots$, $\partial_{0} q\left(\alpha_{m-1}\right)=\partial_{1} q\left(\alpha_{m}\right), \partial_{0} q\left(\alpha_{m}\right)=q\left(x_{E_{i}^{F}}\right)$, where the last equality holds true because $q\left(x_{E_{i}^{F}}\right)=x_{E_{0}^{F}}=q\left(x_{E_{0}^{F}}\right)$.

Let $x, y \in K_{0}^{\text {str }}(Q)$. Let $C_{1}, C_{2}$ be the path components of $\partial Q$ with $x \in C_{1}$ and $y \in C_{2}$. We have constructed sequences of 1-simplices $\alpha_{1}, \ldots, \alpha_{k} \in K_{1}(\partial Q)$ and $\beta_{1}, \ldots, \beta_{l} \in K_{1}(\partial Q)$ such that $\partial_{1} q\left(\alpha_{1}\right)=q\left(x_{C_{1}}\right), \partial_{0} q\left(\alpha_{1}\right)=\partial_{1} q\left(\alpha_{2}\right), \ldots$, $\partial_{0} q\left(\alpha_{k-1}\right)=\partial_{1} q\left(\alpha_{k}\right), \partial_{0} q\left(\alpha_{k}\right)=q(x)$, and $\partial_{1} q\left(\beta_{1}\right)=q\left(x_{C_{2}}\right), \partial_{0} q\left(\beta_{1}\right)=\partial_{1} q\left(\beta_{2}\right)$, $\ldots, \partial_{0} q\left(\beta_{k-1}\right)=\partial_{1} q\left(\beta_{k}\right), \partial_{0} q\left(\beta_{k}\right)=q(y)$. Note that all $q\left(\alpha_{i}\right)$ and $q\left(\beta_{i}\right)$ are either constant or contained in $q\left(K_{1}\left(\partial_{0} Q\right)\right)$.

Let $H:=q_{*}\left(\Pi\left(K\left(\partial_{0} Q\right)\right)\right)$. Define

$$
g:=\left\{q\left(\alpha_{1}\right), q\left(\bar{\alpha}_{1}\right)\right\} \ldots\left\{q\left(\alpha_{k}\right), q\left(\bar{\alpha}_{k}\right)\right\}\left\{q\left(\beta_{l}\right), q\left(\bar{\beta}_{l}\right)\right\} \ldots\left\{q\left(\beta_{1}\right), q\left(\bar{\beta}_{1}\right)\right\} \in H .
$$

(If $k=l=0$, this just means $g=1$.)

We have $g=g^{-1}$ and

$$
g e \in K_{1, q(x) q(y)}^{\mathrm{str}}(Q) \Longleftrightarrow e \in K_{1, q\left(x_{C_{1}}\right) q\left(x_{C_{2}}\right)}^{\mathrm{str}}(Q) .
$$

By construction, the $g$ associated to $x_{E_{i}^{F}}, x_{E_{j}^{F^{\prime}}}$ agrees with the $g$ associated to $x_{E_{0}^{F}}, x_{E_{0}^{F^{\prime}}}$.

We are given $D_{x_{C_{1}} x_{C_{2}}}$ and we want to define $D_{x y}$ such that condition (xiii) is satisfied.

First, if $C_{1} \cap \partial_{1} Q=\varnothing$ or $C_{2} \cap \partial_{1} Q=\varnothing$, then we can fix an arbitrary choice of $D_{x, y}$ satisfying conditions (x)-(xii). (Condition (xiii) is empty in this case.)

So let us assume $C_{1} \cap \partial_{1} Q \neq \varnothing$ and $C_{2} \cap \partial_{1} Q \neq \varnothing$. We note that

$$
q:\left(Q, \partial Q, \partial_{1} Q\right) \rightarrow\left(Q, \partial Q, \partial_{1} Q\right)
$$


is homotopic to the identity as a map of triples, by the construction in Section 5A. This implies that cosets of $\Gamma K_{1, x y}^{\text {str }}(Q) \Gamma$ are in one-to-one correspondence (by applying $q$ ) with those of $\Gamma K_{1, q(x) q(y)}^{\text {str }} \Gamma$. Thus it suffices to describe $q\left(D_{x y}\right) \subset$ $K_{1, q(x) q(y)}^{\mathrm{str}}$.

Let

$$
\Gamma f \Gamma \in \Gamma K_{1, q(x) q(y)}^{\mathrm{str}}(Q) \Gamma
$$

be a double coset. Then the double coset

$$
\Gamma(g f) \Gamma \in \Gamma K_{1, q\left(x_{C_{1}}\right) q\left(x_{C_{2}}\right)}^{\mathrm{str}}(Q) \Gamma
$$

is the image under $q$ of some double coset

$$
\Gamma e^{\prime} \Gamma \in \Gamma K_{1, x_{C_{1}} x_{C_{2}}}^{\mathrm{str}}(Q) \Gamma
$$

Let $e$ be the unique distinguished simplex in $\Gamma e^{\prime} \Gamma$. Then we choose $g q(e)$ to be the distinguished simplex in $\Gamma f \Gamma$. This is possible because $g q(e)$ belongs to the double coset $\Gamma f \Gamma$. Indeed,

$$
q(e) \in \Gamma(g f) \Gamma
$$

means that $q(e)=q_{*}\left(\gamma_{1}\right) g f q_{*}\left(\gamma_{2}\right)$ for some loops $\gamma_{1}$ and $\gamma_{2}$ based at $x_{C_{1}}$ and $x_{C_{2}}$, respectively, and this implies $g q\left(e^{\prime}\right)=q_{*}\left(\gamma_{1}^{\prime}\right) f q_{*}\left(\gamma_{2}^{\prime}\right)$ with

$$
\gamma_{1}^{\prime}:=\left[\bar{\alpha}_{m} * \ldots * \bar{\alpha}_{1} * \gamma_{1} * \alpha_{1} * \ldots * \alpha_{m}\right], \gamma_{2}^{\prime}:=\left[\bar{\beta}_{n} * \ldots * \bar{\beta}_{1} * \gamma_{2} * \beta_{1} * \ldots * \beta_{n}\right] .
$$

This defines $D_{x y}$. By construction, condition (xiv) is satisfied if $e_{1} \in D_{x_{C_{1}} x_{C_{2}}}$. In general, if $e_{1} \in D_{x_{1} y_{1}}$, then we get $e \in D_{x_{C_{1}} x_{C_{2}}}$ and $g_{1} \in H$ with $q\left(e_{1}\right)=g_{1} q(e)$ and $e_{2} \in D_{x_{2} y_{2}}, g_{2} \in H$ with $q\left(e_{2}\right)=g_{2} q(e)$; thus $q\left(e_{2}\right)=g_{2} g_{1}^{-1} q\left(e_{1}\right)$.

Condition (xiii) is implied because $q\left(x_{E_{i}^{F}}\right)=x_{E_{0}^{F}}, q\left(x_{E_{j}^{F^{\prime}}}\right)=x_{E_{0}^{F^{\prime}}}$ and the $g$ associated to $x_{E_{i}^{F}}, x_{E_{j}^{F^{\prime}}}$ agrees with the $g$ associated to $x_{E_{0}^{F}}, x_{E_{0}^{F^{\prime}}}$.

One checks easily that (xi) and (xii) are true for $D_{x y}$, since they are true for $D_{x_{C_{1}} x_{C_{2}}}$.

Definition 5.10. Let $Q, \partial Q, \partial_{0} Q, \partial_{1} Q$ satisfy Assumption I. Let $z=\sum_{i \in I} a_{i} \tau_{i} \in$ $C_{n}^{\text {inf }}(Q)$ be a singular chain and let $\Upsilon$ be the associated simplicial set (for some set of cancellations $\mathscr{C}$ ).

We say that a 0-1 labeling of the elements of the 1-skeleton $\Upsilon_{1}$ is admissible if $\partial e_{1} \cap \partial e_{2}=\varnothing$ for all 1-labeled vertices $e_{1}, e_{2}$.

Lemma 5.11. Let $Q, \partial Q, \partial_{1} Q, \partial_{0} Q$ satisfy Assumption I. Let $q: Q \rightarrow Q$ be given by Construction 5.1.

Let $K_{*}^{\mathrm{str}}(Q) \subset S_{*}(Q)$ satisfy conditions (i)-(viii) from Lemma 5.3, and let $D \subset$ $K_{1}^{\mathrm{str}}(Q)$ be a set of distinguished 1-simplices.

Let $z=\sum_{i \in I} a_{i} \tau_{i} \in C_{*}^{\text {simp,inf }}(K(Q))$ be a relative cycle with

$$
\partial z \in C_{*}^{\operatorname{simp}, \inf }\left(K\left(\partial_{1} Q\right)\right) .
$$


Let a set of cancellations $\mathscr{C}$ for $z$ and a minimal presentation of $\partial z$ be given. Let $\Upsilon, \partial \Upsilon$ be the associated simplicial sets, $\tau:(|\Upsilon|,|\partial \Upsilon|) \rightarrow\left(Q, \partial_{1} Q\right)$ the associated continuous mapping.

Assume that we have an admissible 0-1 labeling of $\Upsilon_{1}$. Then there exists a relative cycle

$$
z^{\prime}=\sum_{i \in I} a_{i} \tau_{i}^{\prime} \in C_{*}^{\text {simp,inf }}\left(K^{\mathrm{str}}(Q), K^{\mathrm{str}}\left(\partial_{1} Q\right)\right)
$$

satisfying the following conditions:

(i) The associated continuous mappings

$$
\tau, \tau^{\prime}:(|\Upsilon|,|\partial \Upsilon|) \rightarrow\left(Q, \partial_{1} Q\right)
$$

are homotopic by a homotopy mapping $|\partial \Upsilon|$ to $\partial Q$.

(ii) If an edge of some $\tau_{i}$ is labeled by 1 , the corresponding edge of $\tau_{i}^{\prime}$ belongs to $D$.

Remark. The homotopy in (i) does not necessarily map $|\partial \Upsilon|$ to $\partial_{1} Q$, but to $\partial Q$.

Proof. First we apply the canonical straightening $\operatorname{str}_{\text {can }}$ from Observation 5.7. The resulting chain $\sum_{i \in I} a_{i} \operatorname{str}_{\text {can }}\left(\tau_{i}\right)$ satisfies (i), but not necessarily (ii).

$\sum_{i \in I} a_{i} \operatorname{str}_{\text {can }}\left(\tau_{i}\right)$ inherits the admissible labeling from $\sum_{i \in I} a_{i} \tau_{i}$. Thus we can, without loss of generality, restrict ourselves to the case that all $\tau_{i}$ belong to $K^{\operatorname{str}}(Q)$.

Let $e \in K_{1}^{\mathrm{str}}(Q)$ be a 1-labeled edge, and set $x=\partial_{1} e \in K_{0}^{\mathrm{str}}(Q), y=\partial_{0} e \in$ $K_{0}^{\mathrm{str}}(Q)$. By Definition 5.8, the coset $\Gamma e \Gamma$ contains a unique distinguished 1simplex $\operatorname{str}(e) \in D_{x y}$. (We use the notation from Definition 5.8; in particular, $\Gamma:=\Omega(\partial Q)$.)

That $\operatorname{str}(e) \in \Gamma e \Gamma$ means $^{6}$ that there are loops $\gamma_{1}, \gamma_{2} \subset \partial Q$ based at $x$ and $y$, respectively, such that $\operatorname{str}(e) \sim \gamma_{1} * e * \gamma_{2}$ rel $\{0,1\}$. There is an obvious homotopy between $e$ and $\gamma_{1} * e * \gamma_{2}$, which moves $\partial_{1} e$ along $\bar{\gamma}_{1}$ and $\partial_{0} e$ along $\gamma_{2}$. (Of course, we change the homotopy class relative boundary, so we cannot keep the endpoints fixed during the homotopy.) If $e$ and/or $\partial_{0} e$ and/or $\partial_{1} e$ have image in $\partial_{1} Q$, then their images remain in $\partial Q$ (and end up in $\partial_{1} Q$ ) during the homotopy.

Using Observation 3.1, the homotopy thus constructed between $e$ and $\operatorname{str}(e)$ can be extended to a homotopy from

$$
\tau:(|\Upsilon|,|\partial \Upsilon|) \rightarrow\left(Q, \partial_{1} Q\right)
$$

to some

$$
\hat{\tau}:(|\Upsilon|,|\partial \Upsilon|) \rightarrow\left(Q, \partial_{1} Q\right),
$$

such that $\hat{\tau}$ is a simplicial map from $\Upsilon$ to $S_{*}(Q)$. (If a 0-labeled edge has one or both vertices adjacent to 1-labeled edges, then the 0-labeled edge just follows the

${ }^{6}$ If $\partial_{0} e, \partial_{1} e \notin \partial_{1} Q$, then $\operatorname{str}(e) \in \Gamma e \Gamma$ means, of course, $\operatorname{str}(e)=e$. Similarly, if only one vertex of $e$ belongs to $\partial_{1} Q$, then only that vertex is moved during the homotopy. 
homotopy of the vertices. Edges labeled with 0 but not adjacent to 1-labeled edges can remain fixed during the homotopy.) The homotopy maps $|\partial \Upsilon|$ to $\partial Q$.

Next we apply homotopies rel boundary to the (already homotoped images of) all 0-labeled edges $f \in K_{1}^{\mathrm{str}}(Q)$, to homotope them to edges in $K_{1}^{\mathrm{str}}(Q)$. If $f$ and/or $\partial_{0} f$ and/or $\partial_{1} f$ have image in $\partial_{1} Q$, then their images remain in $\partial Q$ (and end up in $\partial_{1} Q$ ) during the homotopy.

Now we have a simplicial map $\hat{\tau}: \Upsilon \rightarrow S_{*}(Q)$, such that all 1-simplices are mapped to $K_{1}^{\mathrm{str}}(Q)$, and such that

$$
\hat{\tau}(e) \in D \subset K_{1}^{\mathrm{str}}(Q)
$$

holds for all 1-labeled edges $e$. Then we can, as in the proof of Observation 5.7, by induction on $n$, apply homotopies rel boundary to all $n$-simplices to homotope them into $K_{n}^{\text {str }}(Q)$. Simplices in $\partial_{1} Q$ remain in $\partial Q$ (and end up in $\partial_{1} Q$ ) during the homotopy.

We obtain a homotopy (of pairs), which keeps the 1-skeleton fixed, to a simplicial map

$$
\tau^{\prime}: \Upsilon \rightarrow K^{\mathrm{str}}(Q),
$$

mapping $\partial \Upsilon$ to $K^{\text {str }}\left(\partial_{1} Q\right)$ and satisfying conditions (i) and (ii) of Lemma 5.11.

A somewhat artificial formulation of the conclusion of Lemma 5.11 is that we have constructed a chain map

$$
\text { str : } C_{*}^{\text {simp,inf }}(\Upsilon, \partial \Upsilon) \rightarrow C_{*}^{\text {simp,inf }}\left(K^{\mathrm{str}}(Q), K^{\mathrm{str}}\left(\partial_{1} Q\right)\right) .
$$

Unfortunately, this somewhat artificial formulation can not be simplified because str depends on the chain $\sum_{i \in I} a_{i} \tau_{i}$. That is, we do not get a chain map

$$
\text { str : } C_{*}^{\text {simp,inf }}\left(K(Q), K\left(\partial_{1} Q\right)\right) \rightarrow C_{*}^{\text {simp,inf }}\left(K^{\text {str }}(Q), K^{\text {str }}\left(\partial_{1} Q\right)\right) .
$$

5D. Straightening of crushed cycles. Recall from Section $3 \mathrm{H}$ that $(\cdot) \otimes_{\mathbb{Z} G} \mathbb{Z}$ means the tensor product with the trivial $\mathbb{Z} G$-module $\mathbb{Z}$, that is, the quotient under the $G$-action. We first state obvious generalizations of Observation 5.6 to the case of tensor products with a factor with trivial $G$-action.

Observation 5.12. Let $\left(Q, \partial_{1} Q\right)$ be a pair of topological spaces. Let $G$ be a group acting on a pair $(K, \partial K)$ with $K \subset S_{*}(Q)$ and $\partial K \subset S_{*}\left(\partial_{1} Q\right)$ both closed under face maps.

(i) If

$$
z=\sum_{i \in I} a_{i} \tau_{i} \otimes 1 \in C_{*}^{\text {simp,inf }}(K, \partial K) \otimes_{\mathbb{Z} G} \mathbb{Z}
$$

is a relative cycle, so is

$$
\hat{z}=\sum_{i \in I} \sum_{g \in G} a_{i}\left(g \tau_{i}\right) \in C_{*}^{\text {simp,inf }}(K, \partial K) .
$$


If $\mathscr{C}$ is a sufficient set of cancellations for $z$, there exists a set of cancellations $\widehat{\mathscr{C}}$ for $\hat{z}$ such that $\left(\eta_{1}, \eta_{2}\right) \in \widehat{\mathscr{C}}$ implies $\left(\eta_{1} \otimes 1, \eta_{2} \otimes 1\right) \in \mathscr{C}$.

If $\partial z=\sum_{a, i} c_{a i} \partial_{a} \tau_{i} \otimes 1$ is a minimal presentation for $\partial z$, then

$$
\partial \hat{z}=\sum_{g \in G} \sum_{a, i} c_{a i} \partial_{a}\left(g \tau_{i}\right)
$$

is a minimal presentation for $\hat{z}$.

(ii) Let $\widehat{\Upsilon}, \partial \widehat{\Upsilon}$ be the simplicial sets associated to $\hat{z}$, the sufficient set of cancellations $\widehat{\mathscr{C}}$ and the minimal presentation of $\partial \hat{z}$. They come with an obvious $G$-action. Then we have an associated continuous mapping $\hat{\tau}:(|\widehat{\Upsilon}|,|\partial \Upsilon|) \rightarrow\left(Q, \partial_{1} Q\right)$.

Corollary 5.13. Let $Q, \partial Q, \partial_{1} Q, \partial_{0} Q$ satisfy Assumption I. Let $q: Q \rightarrow Q$ be given by Construction 5.1. Let $K_{*}^{\mathrm{str}}(Q) \subset S_{*}(Q)$ satisfy conditions (i)-(viii) from Lemma 5.3, and let $D \subset K_{1}^{\mathrm{str}}(Q)$ be a set of distinguished 1-simplices.

Let $G:=\Pi\left(K\left(\partial_{0} Q\right)\right)$ with its action on $K^{\mathrm{str}}(Q)$ defined in Observation 5.4, and let $H:=q_{*}(G)$ as defined in Section 5A. Let

$$
\sum_{i \in I} a_{i} \tau_{i} \otimes 1 \in C_{n}^{\text {simp,inf }}\left(K(Q), G K\left(\partial_{1} Q\right)\right) \otimes_{\mathbb{Z} G} \mathbb{Z}
$$

be a relative cycle. Fix a sufficient set of cancellations $\mathscr{b}$ and a minimal presentation for $\partial z$. Let $\widehat{\Upsilon}, \partial \widehat{\Upsilon}$ be defined by Observation 5.12. Assume that we have a $G$-invariant admissible 0-1 labeling of the edges of $\widehat{\Upsilon}$.

Then there is a well-defined chain map

$$
q \circ \operatorname{str}: C_{*}^{\text {simp,inf }}(\widehat{\Upsilon}) \otimes_{\mathbb{Z} G} \mathbb{Z} \rightarrow C_{*}^{\text {simp, inf }}\left(H K^{\text {str }}(Q)\right) \otimes_{\mathbb{Z} H} \mathbb{Z},
$$

mapping $C_{*}^{\text {simp,inf }}(\partial \widehat{\Upsilon}) \otimes_{\mathbb{Z} G} \mathbb{Z}$ to $C_{*}^{\text {simp,inf }}\left(G K^{\text {str }}\left(\partial_{1} Q\right)\right) \otimes_{\mathbb{Z} H} \mathbb{Z}$, satisfying the following conditions:

(i) If $e \in \widehat{\Upsilon}_{1}$ is a 1-labeled edge, $\operatorname{str}(e \otimes 1)=f \otimes 1$, then $f \in D$.

(ii) If $Q$ is an orientable manifold with boundary $\partial Q$, and if

$$
\sum_{i \in I} a_{i} \tau_{i} \otimes 1 \in C_{*}^{\text {simp,inf }}\left(K(Q), G K\left(\partial_{1} Q\right)\right) \otimes_{\mathbb{Z} G} \mathbb{Z}
$$

represents $^{7}$ the image of $[Q, \partial Q] \otimes 1$, then

$$
\sum_{i \in I} a_{i} q \circ \operatorname{str}\left(\tau_{i} \otimes 1\right) \in C_{*}^{\mathrm{simp}, \inf }\left(H K^{\mathrm{str}}(Q), H K^{\mathrm{str}}\left(\partial_{1} Q\right)\right) \otimes_{\mathbb{Z} H} \mathbb{Z}
$$

represents $^{7}$ the image of $[Q, \partial Q] \otimes 1$ and

$$
\partial \sum_{i \in I} a_{i} q \circ \operatorname{str}\left(\tau_{i} \otimes 1\right) \in C_{*}^{\mathrm{simp}, \inf }\left(H K^{\mathrm{str}}\left(\partial_{1} Q\right)\right) \otimes_{\mathbb{Z} H} \mathbb{Z}
$$

represents the image of $[\partial Q] \otimes 1$.

\footnotetext{
${ }^{7}$ See the remark following Lemma 3.6.
} 
Proof. We can apply Lemma 5.11 to the infinite chain $\sum_{i \in I, g \in H} a_{i}\left(g \tau_{i}\right)$ to obtain a chain map str : $C_{*}^{\text {simp,inf }}(\widehat{\Upsilon}) \rightarrow C_{*}^{\text {simp,inf }}\left(K^{\text {str }}(Q)\right)$, given by

$$
\operatorname{str}\left(g \tau_{i}\right):=\left(g \tau_{i}\right)^{\prime} .
$$

The map $q:\left(K^{\mathrm{str}}(Q), K^{\mathrm{str}}\left(\partial_{1} Q\right)\right) \rightarrow\left(K^{\mathrm{str}}(Q), K^{\mathrm{str}}\left(\partial_{1} Q\right)\right)$ is defined by Observation 5.4. (We actually have $q \circ \operatorname{str}\left(g \tau_{i}\right) \in K^{\operatorname{str}}(Q)$. We need $H K^{\mathrm{str}}(Q)$ in the statement of Corollary 5.13 just to have the tensor product well-defined.)

We are going to define $q \circ \operatorname{str}(\sigma \otimes z):=q(\operatorname{str}(\sigma)) \otimes z$ for each $\sigma \in \widehat{\Upsilon}$ and $z \in \mathbb{Z}$. For this to be well-defined, we need this fact:

Claim. For each $\sigma \in K, g \in G$, there exists $h \in H$ with $q(\operatorname{str}(g \sigma))=h q(\operatorname{str}(\sigma))$.

Proof. By condition (viii) from Lemma 5.3 (asphericity of $K^{\text {str }}(Q)$ ), it suffices to check this for the 1-skeleton.

0 -simplices. If $\sigma=v \in S_{0}\left(\partial_{0} Q\right)$ then $v$ and $g v$ belong to the same path component $C$ of $\partial_{0} Q$, hence $\operatorname{str}(v)$ and $\operatorname{str}(g v)$ belong to the same path component $C$. Let $\gamma:[0,1] \rightarrow \partial_{0} Q$ be a path with $\gamma(0)=\operatorname{str}(v), \gamma(1)=\operatorname{str}(g v)$. Let $\gamma^{\prime}$ be the unique 1-simplex in $K\left(\partial_{0} Q\right)$ which is homotopic rel boundary to $\gamma$. Let $g^{\prime}:=\left\{\gamma^{\prime}, \overline{\gamma^{\prime}}\right\} \in G=\Pi\left(K\left(\partial_{0} Q\right)\right)$. Then $g^{\prime} \operatorname{str}(v)=\operatorname{str}(g v)$, which implies $q(\operatorname{str}(g v))=h q(\operatorname{str}(v))$ with $h=q_{*}\left(g^{\prime}\right) \in H$.

If $\sigma=v \notin \partial_{0} Q$, then $g v=v$, hence $q(\operatorname{str}(g v))=q(\operatorname{str}(v))$.

1-simplices. In a first step we prove that for $e \in K_{1}(Q)$ and $g \in G$ we have $\operatorname{str}_{\text {can }}(g e)=g^{\prime} \operatorname{str}_{\text {can }}(e)$ with $g^{\prime} \in G$. Then we show that, if $e \in K_{1}^{\mathrm{str}}(Q)$ and $g \in G$, there exists $h \in H$ with $q(\operatorname{str}(g e))=h q(\operatorname{str}(e))$. Hence altogether we will

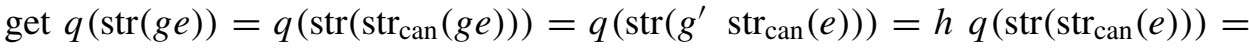
$h q(\operatorname{str}(e))$.

Step 1 . This is basically a case analysis.

First case: If both vertices of $e$ do not belong to $\partial_{0} Q$, then also both vertices of $\operatorname{str}_{\text {can }}(e)$ do not belong to $\partial_{0} Q$, and we have $g e=e, g \operatorname{str}_{\mathrm{can}}(e)=\operatorname{str}(e)$, which implies the conclusion.

Second case: If both vertices of $e$ belong to $\partial_{0} Q$, then $\operatorname{str}_{\text {can }}(e) \sim \alpha_{1} * e * \alpha_{2}$ and $\operatorname{str}_{\text {can }}(g e) \sim \beta_{1} * g e * \beta_{2}$ for some paths $\alpha_{1}, \alpha_{2}, \beta_{1}, \beta_{2}$ in $\partial_{0} Q$. Moreover, by the definition of the action (Section 3.3) we have $g e \sim \gamma_{2} * e * \gamma_{1}$ for some $\gamma_{1}, \gamma_{2} \in K_{1}\left(\partial_{0} Q\right)$. Thus $\operatorname{str}_{\text {can }}(g e) \sim \beta_{1} * \gamma_{1} * \alpha_{1}^{-1} * \operatorname{str}_{\text {can }}(e) * \alpha_{2}^{-1} * \gamma_{2} * \beta_{2}$; in particular $\operatorname{str}_{\mathrm{can}}(g e)=g^{\prime} \operatorname{str}_{\mathrm{can}}(e)$ for some $g^{\prime} \in G$.

Third case: Finally we consider the case that one vertex, say $\partial_{0} e$, belongs to $\partial_{0} Q$, but $\partial_{1} e$ does not. Then we are in the situation of the second case with $\gamma_{2}=1$ and $\alpha_{2}=\beta_{2}$, except that $\alpha_{2}$ is not contained in $\partial_{0} Q$. We get $\operatorname{str}_{\text {can }}(g e) \sim$ $\beta_{1} * \gamma_{1} * \alpha_{1}^{-1} * \operatorname{str}_{\text {can }}(e)$. Since $\beta_{1} * \gamma_{1} * \alpha_{1}^{-1}$ is contained in $\partial_{0} Q$, this implies that $\operatorname{str}_{\mathrm{can}}(g e)=g^{\prime} \operatorname{str}_{\mathrm{can}}(e)$ for some $g^{\prime} \in G$. 
Step 2. Let $e \in K_{1}^{\mathrm{str}}(Q)$.

If $e$ is a 1-labeled edge, with $x=\partial_{1} e, y=\partial_{0} e \in K_{0}^{\mathrm{str}}(Q)$, then we have by condition (xiv) from Definition 5.8 that

$$
q(\operatorname{str}(g e))=h q\left(e_{2}\right)
$$

for some $e_{2} \in D_{x y}$ and some $h \in H$. But $e_{2}$ belongs to the same coset in $\Gamma K_{1}^{\mathrm{str}}(Q) \Gamma$ as $e$; thus $e_{2}=\operatorname{str}(e)$, which proves the claim for $e$.

If $f$ is adjacent to one 1-labeled edge $e$ and $q(\operatorname{str}(g e))=h q(\operatorname{str}(e))$, then $q(\operatorname{str}(g f))=h q(\operatorname{str}(f))$ because the homotopy of $f$ just followed that of $e$, and the homotopy of $g f$ just followed that of $g e$; for example, if $\partial_{1} f=\partial_{1} e$ and $q(\operatorname{str}(g e)) \sim$ $q_{*}(\alpha) * q(\operatorname{str}(e)) * q_{*}(\beta)$ with $\alpha, \beta \in K_{1}\left(\partial_{0} Q\right)$, then $q(\operatorname{str}(g f)) \sim q_{*}(\alpha) * q(\operatorname{str}(f))$. Similarly if $f$ is adjacent to two 1-labeled edges.

Finally, if a 0-labeled straight 1-simplex $f$ is not adjacent to a 1-labeled edge, we have $\operatorname{str}(f)=f$ and $\operatorname{str}(g f)=g f$, which implies that $\operatorname{str}(g f)=g \operatorname{str}(f)$ and $q(\operatorname{str}(g f))=q_{*}(g) \operatorname{str}(f)$.

This concludes the proof of the claim.

Thus $q \circ$ str is well-defined; by Lemma 5.11, it satisfies the equation in part (i) of our corollary. To prove part (ii), we first observe that, if $\sum_{i \in I} a_{i} \tau_{i}$ represents $[Q, \partial Q]$, then, by Observation 5.6(c) and condition (i) from Lemma 5.11 (together with $q \sim$ id), the element

$$
\sum_{i \in I} a_{i} q \circ \operatorname{str}\left(\tau_{i}\right)=\sum_{i=1}^{r} a_{i} q\left(\tau_{i}^{\prime}\right)
$$

represents $[Q, \partial Q]$ and the claim follows. Thus it suffices to check: if $\sum_{i \in I} a_{i} \tau_{i} \otimes 1$ is (relatively) homologous to $\sum_{j \in J} b_{j} \kappa_{j} \otimes 1$, then $q \circ \operatorname{str}\left(\sum_{i \in I} a_{i} \tau_{i} \otimes 1\right)$ is (relatively) homologous to $q \circ \operatorname{str}\left(\sum_{j \in J} b_{j} \kappa_{j} \otimes 1\right)$.

So let

$$
\sum_{i \in I} a_{i} \tau_{i} \otimes 1-\sum_{j \in J} b_{j} \kappa_{j} \otimes 1=\partial \sum_{k \in K} c_{k} \eta_{k} \otimes 1 \bmod C_{*}^{\operatorname{simp}, \text { inf }}\left(G K\left(\partial_{1} Q\right)\right) \otimes_{\mathbb{Z} G} \mathbb{Z}
$$

for some chain $\sum_{k \in K} c_{k} \eta_{k} \otimes 1 \in C_{*}^{\text {simp,inf }}(K(Q)) \otimes_{\mathbb{Z} G} \mathbb{Z}$. In complete analogy with Lemma 5.11, we may extend str to the simplicial set built by the $g \eta_{k}$, their faces and degenerations, and obtain a singular chain $q\left(\operatorname{str}\left(\sum_{k \in K} c_{k} \eta_{k}\right)\right)$ with boundary

$$
\begin{aligned}
\partial q \circ \operatorname{str}\left(\sum_{k \in K} c_{k} \eta_{k}\right)=q \circ \operatorname{str}\left(\sum_{i \in I} a_{i} \tau_{i} \otimes 1\right)-q \circ \operatorname{str}\left(\sum_{j \in J} b_{j} \kappa_{j} \otimes 1\right) \\
\\
\bmod C_{*}^{\operatorname{simp}, \inf }\left(H K^{\operatorname{str}}\left(\partial_{1} Q\right)\right) \otimes_{\mathbb{Z} H} \mathbb{Z} .
\end{aligned}
$$

This gives the first claim of (ii). The second claim of (ii) follows because $\partial$ maps $[Q, \partial Q]$ to $[\partial Q]$. 


\section{E. Removal of 0-homologous chains.}

Definition 5.14. Let $Q$ be an $n$-dimensional compact manifold with boundary $\partial Q$. We define rmv : $S_{*}(Q) \rightarrow S_{*}(Q)$ by

$$
\operatorname{rmv}(\sigma)= \begin{cases}0 & \text { if } \sigma \text { is weakly degenerate (Definition 5.2), } \\ \sigma & \text { else. }\end{cases}
$$

Lemma 5.15. Assume that $Q$ is a n-dimensional compact manifold with boundary $\partial Q$. Let $K_{*}^{\mathrm{str}}(Q) \subset S_{*}(Q)$ satisfy conditions (i)-(viii) from Lemma 5.3. Then the map from $C_{*}^{\mathrm{simp}}\left(K^{\mathrm{str}}(Q), K^{\mathrm{str}}\left(\partial_{0} Q\right) \cup K^{\mathrm{str}}\left(\partial_{1} Q\right)\right)$ to itself defined by

$$
\operatorname{rmv}([\sigma]):=[\operatorname{rmv}(\sigma)]
$$

is a well-defined chain map. Moreover, if

$$
\sum_{j=1}^{r} a_{j} \tau_{j} \in C_{*}^{\mathrm{simp}}\left(K^{\mathrm{str}}(Q), K^{\mathrm{str}}\left(\partial_{0} Q\right) \cup K^{\mathrm{str}}\left(\partial_{1} Q\right)\right) \subset C_{*}^{\mathrm{sing}}(Q, \partial Q)
$$

represents $[Q, \partial Q]$, then $\sum_{j=1}^{r} a_{j} \operatorname{rmv}\left(\tau_{j}\right)$ represents $[Q, \partial Q]$.

Proof. If $\sigma \in K^{\operatorname{str}}\left(\partial_{0} Q\right) \cup K^{\operatorname{str}}\left(\partial_{1} Q\right)$, then $\operatorname{rmv}(\sigma) \in K^{\operatorname{str}}\left(\partial_{0} Q\right) \cup K^{\operatorname{str}}\left(\partial_{1} Q\right)$; thus rmv is well-defined. We next prove it is a chain map.

Assume that $\operatorname{rmv}(\sigma)=0$. If $\sigma$ has image in $\partial Q$, then $\operatorname{rmv}(\sigma)$ and $\operatorname{rmv}(\partial \sigma)$ both vanish; thus $\partial \operatorname{rmv}(\sigma)=\operatorname{rmv}(\partial \sigma)$.

If some edge $e$ of $\sigma$, say connecting the $i$-th and $j$-th vertices, is a constant loop, then all faces of $\sigma$ except possibly $\partial_{i} \sigma$ and $\partial_{j} \sigma$ have a constant edge. Thus $\operatorname{rmv}\left(\partial_{k} \sigma\right)=0$ if $k \notin\{i, j\}$. Moreover, since $e$ is constant, corresponding edges of $\partial_{i} \sigma$ and $\partial_{j} \sigma$ are homotopic rel boundary and thus agree (possibly up to orientation) by condition (v) from Lemma 5.3. By induction on the dimension of subsimplices we get, again using condition (v) from Lemma 5.3, that $\partial_{i} \sigma=(-1)^{i-j} \partial_{j} \sigma$. Altogether we get $\operatorname{rmv}(\partial \sigma)=0$; thus $\partial \operatorname{rmv}(\sigma)=\operatorname{rmv}(\partial \sigma)$.

Assume that $\operatorname{rmv}(\sigma)=\sigma$. Since no edge of $\sigma$ is a constant loop, of course also no edge of a face $\partial_{i} \sigma$ is a constant loop. If the image of $\partial_{i} \sigma$ is not contained in $\partial Q$, this implies $\operatorname{rmv}\left(\partial_{i} \sigma\right)=\partial_{i} \sigma=\partial_{i} \operatorname{rmv}(\sigma)$. If $\partial_{i} \sigma$ has image in $\partial Q$, then of course $\left[\partial_{i} \sigma\right]=[0]=\left[\partial_{i} \operatorname{rmv}(\sigma)\right]$, which implies $\operatorname{rmv}\left(\partial_{i} \sigma\right)=\partial_{i} \operatorname{rmv}(\sigma)$.

Now we prove that rmv sends relative fundamental cycles to relative fundamental cycles. Let $\sum_{j=1}^{r} a_{j} \tau_{j}$ be a straight relative cycle representing the relative homology class $[Q, \partial Q]$. We denote by $J_{1} \subset\{1, \ldots, r\}$ the indices of those $\tau_{j}$ which have a constant edge. The sum $\sum_{j \in J_{1}} a_{j} \tau_{j}$ is a relatively 0-homologous relative cycle. Indeed, each face of $\partial_{i} \tau_{k}$ not contained in $\partial Q$ has to cancel against some face of some $\tau_{l}$, because $\sum_{j=1}^{r} a_{j} \tau_{j}$ is a relative cycle. If $\partial_{i} \tau_{k}$ is degenerate, then necessarily $l \in J_{1}$. Moreover, if $\tau_{k}$ is degenerate and $\partial_{i} \tau_{k}$ is nondegenerate, it follows from the earlier part of the proof that $\partial_{i} \tau_{k}$ cancels against some $\partial_{j} \tau_{k}$. 
Thus $\sum_{j \in J_{1}} a_{j} \tau_{j}$ represents some relative homology class. The isomorphism $H_{n}\left(C_{*}^{\text {sing }}(Q, \partial Q)\right) \rightarrow \mathbb{R}$ is given by pairing with the volume form of an arbitrary Riemannian metric. After smoothing the relative cycle, we can apply Sard's lemma, and conclude that degenerate simplices have volume 0 . Thus $\sum_{j \in J_{1}} a_{j} \tau_{j}$ is 0-homologous.

We denote by $J_{2} \subset\{1, \ldots, r\}$ the indices of those $\tau_{j}$ which are contained in $\partial Q$. For $j \in J_{2}$ we have $\left[\tau_{j}\right]=[0] \in C_{*}^{\operatorname{sing}}(Q, \partial Q)$.

Thus $\sum_{j \notin J_{1} \cup J_{2}} a_{j} \tau_{j}$ is another representative of the homology class $[Q, \partial Q]$. But, by Definition 5.14, it also represents $(\mathrm{rmv})_{*}([Q, \partial Q])$.

Consider a subgroup $H \subset \Pi(K(A))$ for some $A \subset \partial Q$. For instance, $A=q\left(\partial_{0} Q\right)$ in the setting of Construction 5.1, and $H=q_{*}\left(\Pi\left(K\left(\partial_{0} Q\right)\right)\right) \subset \Pi(K(A))$.

A 1-simplex $e$ is a constant loop if and only if he is a constant loop for all $h \in H$. This implies that a simplex $\sigma$ is degenerate if and only if $h \sigma$ is degenerate for all $h \sigma$. Moreover, $H$ maps simplices in $\partial Q$ to simplices in $\partial Q$. Thus $\operatorname{rmv}(\sigma)=0$ if and only if $\operatorname{rmv}(h \sigma)=0$ for all $h \in H$, that is, rmv is well defined on $C_{*}^{\text {simp,inf }}\left(H K^{\text {str }}(Q)\right) \otimes_{\mathbb{Z} H} \mathbb{Z}$ for each subgroup $H$.

Lemma 5.16. Assume that $Q$ is a n-dimensional compact manifold with boundary $\partial Q$. Let the assumptions of Corollary 5.13 be satisfied. Then we can extend $\mathrm{rmv}$ to a well-defined chain map from $\left(H K^{\mathrm{str}}(Q), H K^{\mathrm{str}}\left(\partial_{1} Q\right)\right) \otimes_{\mathbb{Z} H} \mathbb{Z}$ to itself by defining

$$
\operatorname{rmv}(\sigma \otimes z)= \begin{cases}0 & \text { if } \operatorname{rmv}(\sigma)=0, \\ \sigma \otimes z & \text { else. }\end{cases}
$$

Moreover, if $\sum_{j \in J} a_{j} \tau_{j} \otimes 1 \in C_{*}^{\mathrm{simp}, \text { inf }}\left(H K^{\mathrm{str}}(Q), H K^{\mathrm{str}}\left(\partial_{1} Q\right)\right) \otimes_{\mathbb{Z} H} \mathbb{Z}$ represents the image of $[Q, \partial Q] \otimes 1$, then $\sum_{\in J} a_{j} \operatorname{rmv}\left(\tau_{j} \otimes 1\right)$ represents the image of $[Q, \partial Q] \otimes 1$.

Proof. Well-definedness of rmv follows from the remark before Lemma 5.16. The same proof as for Lemma 5.15 shows that rmv is a chain map.

If $\sum_{j=1}^{r} a_{j} \tau_{j}$ represents [ $Q, \partial Q$ ], the second claim follows from Lemma 5.15. If $\sum_{j \in J} a_{j} \tau_{j} \otimes 1$ is homologous to $\sum_{i=1}^{s} b_{i} \kappa_{i} \otimes 1$ and $\sum_{i=1}^{s} b_{i} \kappa_{i}$ represents $[Q, \partial Q]$, then, because rmv is a chain map, $\operatorname{rmv}\left(\sum_{j \in J} a_{j} \tau_{j} \otimes 1\right)$ and $\operatorname{rmv}\left(\sum_{i=1}^{s} b_{i} \kappa_{i} \otimes 1\right)$ are homologous, which implies the second claim.

The proof of Theorem 1.1 will pursue the idea of straightening a given cycle in such a way that many simplices either become weakly degenerate or will have an edge in $\partial_{0} Q$. In the first case, they will disappear after application of rmv. In the second case, they disappear in view of the following observation, which is a variant of an argument used in [Gromov 1982].

Lemma 5.17. (a) Let Assumption I be satisfied for a manifold $Q$ and consider the action of $G=\Pi\left(K\left(\partial_{0} Q\right)\right)$ on $K(Q)$. Let $\sigma \in K(Q)$ be a simplex. If 
$\operatorname{str}(\sigma)$ has an edge in $\partial_{0} Q$, then

$$
\operatorname{str}(\sigma \otimes 1)=0 \in C_{*}^{\operatorname{simp}, \inf }(K(Q)) \otimes_{\mathbb{Z} G} \mathbb{Z} .
$$

(b) If $q: Q \rightarrow Q$ is given by Construction 5.1, $H=q_{*}(G)$, and $\sigma \in K(Q)$ is a simplex such that $q(\operatorname{str}(\sigma))$ has an edge in $q\left(\partial_{0} Q\right)$, then

$$
q(\operatorname{str}(\sigma \otimes 1))=0 \in C_{*}^{\operatorname{simp}, \inf }(K(Q)) \otimes_{\mathbb{Z} H} \mathbb{Z} .
$$

Proof. (a) Let $\gamma$ be the edge of $\operatorname{str}(\sigma)$ with image in $\partial_{0} Q$. Then $g=\{\gamma, \bar{\gamma}\}$ is an element of $G=\Pi\left(K\left(\partial_{0} Q\right)\right)$ and $g \operatorname{str}(\sigma)=\overline{\operatorname{str}(\sigma)}$. In the simplicial chain complex $C_{*}^{\text {simp,inf }}(K(Q))$, one has $\overline{\operatorname{str}(\sigma)}=-\operatorname{str}(\sigma)$. Thus $g \operatorname{str}(\sigma)=-\operatorname{str}(\sigma)$, which implies $\operatorname{str}(\sigma \otimes 1)=\operatorname{str}(\sigma) \otimes 1=0$.

(b) Let $\gamma$ be the edge of $q(\operatorname{str}(\sigma))$ with image in $q\left(\partial_{0} Q\right)$. Let $\gamma^{\prime}$ be the corresponding edge of $\operatorname{str}(\sigma)$. Let $g=\left\{\gamma^{\prime}, \bar{\gamma}^{\prime}\right\} \in G$ and $h=q_{*}(g)=\{\gamma, \bar{\gamma}\} \in H$. The same argument as in (a) shows $h q(\operatorname{str}(\sigma))=-q(\operatorname{str}(\sigma))$.

\section{Proof of Main Theorem}

As discussed in the introduction, before tackling the proof of Theorem 1.1 in full generality, we prove some particular cases as motivation.

Example 6.1. $M$ is a connected, orientable, hyperbolic $n$-manifold, $F$ is an orientable, geodesic $(n-1)$-submanifold, and $Q=\overline{M-F}$. For simplicity we assume that $M$ and $F$ are closed; thus $Q$ is a hyperbolic manifold with geodesic boundary $\partial_{1} Q \neq \varnothing$, and $\partial_{0} Q=\varnothing$.

Outline of proof that $\|M\|_{F}^{\text {norm }} \geq\|\partial Q\| /(n+1)$. Start with a fundamental cycle $\sum_{i=1}^{r} a_{i} \sigma_{i}$ of $M$ such that $\sigma_{1}, \ldots, \sigma_{r}$ are normal to $F$. Since we want to consider laminations without isolated leaves, we replace $F$ by a trivially foliated product neighborhood $\mathscr{F}$. We can assume after a suitable homotopy that each component of $\sigma_{i}^{-1}(\partial Q)$ either contains no vertex of $\Delta^{n}$ or consists of exactly one vertex, and that each vertex of $\Delta^{n}$ belongs to $\sigma_{i}^{-1}(\mathscr{F})$, for $i=1, \ldots, r$.

Each $\sigma_{i}^{-1}(Q)$ consists of polytopes, which can be further triangulated (without introducing new vertices) in a coherent way (i.e., such that boundary cancellations between different $\sigma_{i}$ 's will remain) into $\tau_{i 1}, \ldots, \tau_{i s(i)}$.

The sum $\sum_{i=1}^{r} a_{i}\left(\tau_{i 1}+\cdots+\tau_{i s(i)}\right)$ is a relative fundamental cycle for $Q$.

For each $\sigma_{i}$, preimages of the boundary leaves of $\mathscr{F}_{F}$ cut $\Delta^{n}$ into regions which we color with black (components of $\sigma_{i}^{-1}(\mathscr{F})$ ) and white (components of $\sigma_{i}^{-1}(Q)$ ). If $\sigma_{i}^{-1}(\partial Q)$ contains vertices, these vertices are colored black. This is a canonical coloring (Definition 4.5). 
The edges of the simplices $\tau_{i, j}$ fall into two classes: "old edges", i.e., subarcs of edges of $\sigma_{i}$, and "new edges", which are contained in the interior of some subsimplex of $\sigma_{i}$ of dimension $\geq 2$.

We label the edges of $\tau_{i j}$ in such a way that old edges are labeled 1 and new edges are labeled 0. This is an admissible labeling (Definition 5.10). With this labeling, we apply the straightening procedure ${ }^{8}$ from Section 5 to get a straight cycle $\sum_{i=1}^{r} a_{i}\left(\operatorname{str}\left(\tau_{i 1}\right)+\cdots+\operatorname{str}\left(\tau_{i s(i)}\right)\right)$. (Thus old edges are straightened to distinguished 1-simplices.)

After straightening we apply the map rmv from Section 5D to remove all weakly degenerate simplices (simplices contained in $\partial Q$ or having a constant edge). By Lemma 5.15, this does not change the homology class. In particular, the boundary of the relative cycle, $\partial \sum_{i, j} a_{i} \operatorname{rmv}\left(\operatorname{str}\left(\tau_{i j}\right)\right)$ still represents the fundamental class $[\partial Q]$ of $\partial Q$.

Claim. For each $\sigma_{i}$, after straightening there remain at most $n+1$ faces of nondegenerate simplices $\operatorname{str}\left(\tau_{i j}\right)$ contributing to $\partial \sum_{i, j} a_{i} \operatorname{rmv}\left(\operatorname{str}\left(\tau_{i j}\right)\right)$.

Proof of claim. In view of Lemma 4.13, it suffices to show a subclaim: If, for a fixed $i$, the faces $T_{1}=\partial_{k_{1}} \tau_{i j_{1}}$ and $T_{2}=\partial_{k_{2}} \tau_{i j_{2}}$ of $\tau_{i j_{1}}$ and $\tau_{i j_{2}}$ have a white-parallel arc (Definition 4.9), then $\operatorname{rmv}\left(\operatorname{str}\left(\tau_{i j_{1}}\right)\right)$ and $\operatorname{rmv}\left(\operatorname{str}\left(\tau_{i j_{2}}\right)\right)$ vanish. In particular the corresponding straightened faces ${ }^{9} \operatorname{str}\left(T_{1}\right), \operatorname{str}\left(T_{2}\right)$ do not occur (with nonzero coefficient) in $\partial \sum_{i, j} \operatorname{rmv}\left(\operatorname{str}\left(\tau_{i j}\right)\right)$.

To prove the subclaim, let $W$ be the white region of $\Delta^{n}$ containing $T_{1}$ and $T_{2}$ in its boundary. By the assumption of the subclaim, there is a white square bounded by two $\operatorname{arcs} e_{1} \subset T_{1}$, $e_{2} \subset T_{2}$ and two arcs $f_{1}, f_{2}$ which are subarcs of edges of $\Delta^{n}$. (The square is a formal sum of two triangles, $U_{1}+U_{2}$, which are 2-dimensional faces of some $\tau_{i j}$ 's.)

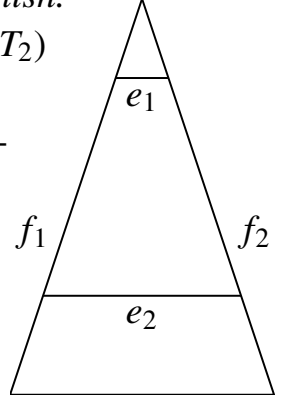

We want to show that all edges of $\operatorname{str}\left(\tau_{i j_{1}}\right)$ belong to $S_{1}^{\text {str }}(\partial Q)$. Note that $T_{1}, T_{2} \subset$ $\partial W$ are mapped to $\partial Q$. Let $x_{1}, x_{2} \in S_{0}^{\mathrm{str}}(Q)$ be the unique elements of $S_{0}^{\mathrm{str}}(Q)$ in the same connected component $C_{1}, C_{2}$ of $\partial Q$ as $\sigma_{i}\left(T_{1}\right)$ and $\sigma_{i}\left(T_{2}\right)$, respectively. In particular $\partial_{0} \operatorname{str}\left(e_{1}\right)=x_{1}=\partial_{1} \operatorname{str}\left(e_{1}\right)$ and $\partial_{0} \operatorname{str}\left(e_{2}\right)=x_{2}=\partial_{1} \operatorname{str}\left(e_{2}\right)$. Thus $e_{1}$ and $e_{2}$ are straightened to loops $\operatorname{str}\left(e_{1}\right)$ and $\operatorname{str}\left(e_{2}\right)$ based at $x_{1}$ and $x_{2}$, respectively. The straightenings $\operatorname{str}\left(f_{1}\right), \operatorname{str}\left(f_{2}\right)$ of the other two arcs connect $x_{1}$ to $x_{2}$, and they are distinguished 1-simplices because they arise as straightenings of old edges. Thus $\operatorname{str}\left(f_{1}\right)=\operatorname{str}\left(f_{2}\right)$, by uniqueness of distinguished 1 -simplices in each coset

\footnotetext{
${ }^{8}$ Under the assumptions of Example 6.1, straight simplices can be chosen to be the totally geodesic simplices with vertices in $S_{0}^{\text {str }}(Q)$. Distinguished simplices are chosen according to Observation 5.9.

${ }^{9}$ For a subsimplex $T$ of an affine subset $S \subset \Delta^{n}$ we get a singular simplex $\left.\sigma_{i}\right|_{T}$ by restricting $\sigma_{i}$ to $T$. We denote by $\operatorname{str}(T)$ the straightening of $\left.\sigma_{i}\right|_{T}$.
} 
$\Gamma K_{1}^{\text {str }}(Q) \Gamma$ of $\Gamma=\Omega(\partial Q)$. This is why we have performed the straightening construction in Section 5 such that there should be only one distinguished 1-simplex, in each coset, for any given pair of connected components.

This means that the square is straightened to a cylinder.

But $(Q, \partial Q)$ is acylindrical; thus either both $\operatorname{str}\left(e_{1}\right)$ and $\operatorname{str}\left(e_{2}\right)$ are constant (in which case $\left.\operatorname{rmv}\left(\operatorname{str}\left(\tau_{i j_{1}}\right)\right)=\operatorname{rmv}\left(\operatorname{str}\left(\tau_{i j_{2}}\right)\right)=0\right)$, or the cylinder must be homotopic into $\partial Q$. In the latter case, $\operatorname{str}\left(f_{1}\right)$ must be homotopic into, and therefore contained in, $\partial Q$. In particular, $\partial_{0} \operatorname{str}\left(f_{1}\right)$ and $\partial_{1} \operatorname{str}\left(f_{1}\right)$ belong to the same component of $\partial Q$. This implies $\partial_{0} \operatorname{str}\left(f_{1}\right)=\partial_{1} \operatorname{str}\left(f_{1}\right)$. Since $\operatorname{str}\left(f_{1}\right)$ is a distinguished 1-simplex, this implies that $\operatorname{str}\left(f_{1}\right)$ is constant.

Let $P_{1}, P_{2}$ be the affine planes whose intersections with $\Delta^{n}$ contain $T_{1}$ and $T_{2}$, respectively. There is an $\operatorname{arc} f_{1}$ connecting $P_{1} \cap \Delta^{n}$ to $P_{2} \cap \Delta^{n}$ such that $\operatorname{str}\left(f_{1}\right)$ is contained in $\partial Q$. This implies that for each other arc $f$ connecting $P_{1} \cap \Delta^{n}$ to $P_{2} \cap \Delta^{n}$ its straightening $\operatorname{str}(f)$ must be homotopic into, and therefore contained in, $\partial Q$.

If $P_{1}$ and $P_{2}$ are of the same type, then all edges of $\operatorname{str}\left(\tau_{i j_{1}}\right)$ connect $P_{1} \cap \Delta^{n}$ to $P_{2} \cap \Delta^{n}$; hence all edges of $\operatorname{str}\left(\tau_{i j_{1}}\right)$ belong to $S_{1}^{\text {str }}(\partial Q)$. If $P_{1}$ and $P_{2}$ are not of the same type, the existence of a parallel arc implies that at least one of them, say $P_{1}$, must be of type $\left\{0 a_{1} \ldots a_{k}\right\}$ with $k \notin\{0, n-1\}$. Then, if $P_{3}$ is any other plane bounding $W$, it follows from Corollary 4.8 that $P_{3}$ has a white-parallel arc with $P_{1}$. Repeating the argument in the last paragraph with $P_{1}$ and $P_{3}$ in place of $P_{1}$ and $P_{2}$, we conclude that for each arc $f$ connecting $P_{1} \cap \Delta^{n}$ to $P_{3} \cap \Delta^{n}$ its straightening $\operatorname{str}(f)$ must be homotopic into, and therefore contained in, $\partial Q$. Hence, for each $\tau_{i j_{1}}$ in the chosen triangulation of $W$, its 1-skeleton is straightened into $\partial Q$.

Since straight simplices $\sigma$ (of dimension $\geq 2$ ) with $\partial \sigma$ in the geodesic boundary $\partial Q$ must be in $\partial Q$, this implies by induction that the $k$-skeleton $\operatorname{of} \operatorname{str}\left(\tau_{i j_{1}}\right)$ is in $\partial Q$ for each $k$. In particular, $\operatorname{str}\left(\tau_{i j_{1}}\right) \in S_{n}^{\mathrm{str}}(\partial Q)$. Hence $\operatorname{rmv}\left(\operatorname{str}\left(\tau_{i j_{1}}\right)\right)=0$. This proves the subclaim.

By Lemma 4.13, the subclaim implies the claim.

Since $\sum_{i=1}^{r} a_{i} \partial \sum_{j} \operatorname{rmv}\left(\operatorname{str}\left(\tau_{i j}\right)\right)$ represents the fundamental class [ $\left.\partial Q\right]$, we conclude that $\|\partial Q\| \leq(n+1) \sum_{i=1}^{r}\left|a_{i}\right|$, as desired.

The simplifications of Example 6.1 in comparison to the general proof below are essentially all due to the fact that $\partial_{0} Q=\varnothing$. In the next example, if $F$ is not geodesic, then $Q \neq N$ and thus $\partial_{0} Q \neq \varnothing$ (even though $\partial M$ and $\partial F$ are both empty). Thus the generalization to $\partial_{0} Q \neq \varnothing$ would be necessary even if one only wanted to consider closed manifolds $M$ and $F$.

Example 6.2. $M$ is a connected, closed, hyperbolic 3-manifold, $F \subset M$ a closed, incompressible surface, $N=\overline{M-F}, Q=\operatorname{Guts}(N)$. 
Outline of proof that $\|M\|_{F}^{\text {norm }} \geq \frac{1}{4}\|\partial Q\|$. Start with a fundamental cycle $\sum_{i=1}^{r} a_{i} \sigma_{i}$ of $M$, such that $\sigma_{1}, \ldots, \sigma_{r}$ are normal to $F$. As in Example 6.1 we get a relative fundamental cycle $\sum_{i=1}^{r} a_{i}\left(\tau_{i 1}+\cdots+\tau_{i s(i)}\right)$ of $N$. We cannot apply the argument from Example 6.1 to $N$ because $N$ is not acylindrical. Therefore we would like to work with a relative fundamental cycle for the acylindrical manifold $Q$.

$N$ is aspherical. Using Lemma 3.2, we can assume that all $\tau_{i j}$ belong to $K(N)$. Then we can apply the retraction $r$ from Lemma 3.5. Since $r$ is only defined after tensoring with $\mathbb{Z}$ over $\mathbb{Z} G$, we get $r\left(\tau_{i j} \otimes 1\right)=\kappa_{i j} \otimes 1$ with $\kappa_{i j} \in K(Q)$ only determined up to choosing one $\kappa_{i j}$ in its $G$-orbit.

Since $Q$ is aspherical, we have $K(Q)=\widehat{K}(Q)$, that is, the $\kappa_{i j}$ can be considered as simplices in $Q$ and we can apply Lemma 3.6(b) to obtain a fundamental cycle for $\partial Q$.

The rest of the proof basically boils down to copying the proof of Example 6.1, with $\tau_{i j}$ replaced by $\kappa_{i j}$; but taking care of the ambiguity in the choice of $\kappa_{i j}$. The details can be found in the full-fledged proof of the theorem we're about to give.

Proof of Theorem 1.1. The theorem is trivially true if $n=1$. Hence we assume $n \geq 2$.

If $\partial_{1} Q$ is empty, the equality $\partial Q=\partial_{0} Q$ and the amenability of $\pi_{1} \partial_{0} Q$ would imply $\|\partial Q\|=0$, and Theorem 1.1 would be trivially true. Hence we assume $\partial_{1} Q \neq \varnothing$. In particular, $Q$ satisfies Assumption I from Section 5.

Consider a relative cycle $\sum_{i=1}^{r} a_{i} \sigma_{i}$, representing $[M, \partial M]$, such that $\sigma_{1}, \ldots, \sigma_{r}$ are normal to $\mathscr{F}$. Our aim is to show that

$$
\sum_{i=1}^{r}\left|a_{i}\right| \geq \frac{1}{n+1}\|\partial Q\|
$$

Define

$$
N=\overline{M-\mathscr{F}} .
$$

Since each $\sigma_{i}$ is normal to $\mathscr{F}$, we have for each $i=1, \ldots, r$ that, after application of a simplicial homeomorphism $h_{i}: \Delta^{n} \rightarrow \Delta^{n}$, the image of $\sigma_{i}^{-1}(N)$ consists of polytopes, which can each be further triangulated in a coherent way (i.e., such that boundary cancellations between different $\sigma_{i}$ 's will remain) into simplices $\theta_{i j}$, with $j \in \hat{J}_{i}$. (It is possible that $\left|\hat{J}_{i}\right|=\infty$, because $N$ may be noncompact.) We choose these triangulations of the $\sigma_{i}^{-1}(N)$ to be minimal (Definition 4.9); that is, we do not introduce new vertices. (Compatible minimal triangulations of the $\sigma_{i}^{-1}(N)$ do exist: one starts with common minimal triangulations of the common faces and extends them to minimal triangulations of each polytope.)

Because boundary cancellations are preserved, we see that $\sum_{i=1}^{r} a_{i} \sum_{j \in \hat{J}_{i}} \theta_{i j}$ is a countable (possibly infinite) relative cycle representing the fundamental class $[N, \partial N]$ in the sense of Section 3B. 
We fix a sufficient set of cancellations $\mathscr{C}^{M}$ for the relative cycle $\sum_{i=1}^{r} a_{i} \sigma_{i}$, in the sense of Definition 5.5. This induces a sufficient set of cancellations $\mathscr{C}^{N}$ for the relative cycle $\sum_{i=1}^{r} \sum_{j \in \hat{J}_{i}} a_{i} \theta_{i j}$.

If $\partial M$ is a leaf of $\mathscr{F}$, then all faces of $z$ contributing to $\partial z$ are contained in $\partial N$. We call these faces exterior faces. We can assume that, for each $i$,

- each component of $\sigma_{i}^{-1}(\partial N)$ either contains no vertex of $\Delta^{n}$, or consists of exactly one vertex, or consists of an exterior face, and

- and each vertex of $\Delta^{n}$ belongs to $\sigma_{i}^{-1}(\mathscr{F})$.

Indeed, by a small homotopy of the relative fundamental cycle $\sum_{i=1}^{r} a_{i} \sigma_{i}$, preserving normality, we can obtain that no component of $\sigma_{i}^{-1}(\partial N)$ contains a vertex of $\Delta^{n}$, except for exterior faces. Afterwards, if some vertices of $\sum_{i=1}^{r} a_{i} \sigma_{i}$ do not belong to $\mathscr{F}$, we may homotope a small neighborhood of the vertex, until the vertex (and no other point of the neighborhood) meets $\partial N$. This, of course, preserves normality to $\mathscr{F}$.

Since each $\sigma_{i}$ is normal to $\mathscr{F}$, in particular each $\sigma_{i}$ is normal to the union of boundary leaves

$$
\partial_{1} N:=\overline{\partial N-(\partial M \cap \partial N)} .
$$

Thus for each $\sigma_{i}$, after application of a simplicial homeomorphism $h_{i}: \Delta^{n} \rightarrow \Delta^{n}$, the image of $\sigma_{i}^{-1}\left(\partial_{1} N\right)$ consists of a (possibly infinite) set

$$
Q_{1}, Q_{2}, \ldots \subset \Delta^{n}
$$

such that

$$
Q_{i}=P_{i} \cap \Delta^{n}
$$

for some affine hyperplanes $P_{1}, P_{2}, \ldots$ We define a coloring by declaring that (images under $h_{i}$ of) components of

$$
\sigma_{i}^{-1}(\operatorname{int}(N)):=\sigma_{i}^{-1}\left(N-\partial_{1} N\right)
$$

are colored white and (images under $h_{i}$ of) components of $\sigma_{i}^{-1}(\mathscr{F})$ are colored black. (In particular, all $Q_{i}$ are colored black.) Since we assume that all vertices of $\Delta^{n}$ belong to $\sigma_{i}^{-1}(\mathscr{F})$, and since each boundary leaf is adjacent to at least one component of $\sigma_{i}^{-1}(\operatorname{int}(N))$, this is a canonical coloring (Definition 4.5).

By Lemma 3.2(a), we can homotope the relative cycle $\sum_{i=1}^{r} \sum_{j \in \hat{J}_{i}} \theta_{i j}$, which belongs to $C_{n}^{\inf }(N, \partial N)$, to a relative cycle

$$
\sum_{i=1}^{r} a_{i} \sum_{j \in \hat{J}_{i}} \hat{\theta}_{i j}
$$

such that each $\hat{\theta}_{i j}$ is a simplex of $\widehat{K}(N)$, as defined in Section $3 \mathrm{~B}$, and such that 
the boundary $\partial \sum_{i=1}^{r} \sum_{j \in \hat{J}_{i}} \theta_{i j}$ is homotoped into $\widehat{K}(\partial N)$. Then consider

$$
\sum_{i=1}^{r} \sum_{j \in \hat{J}_{i}} a_{i} \tau_{i j}:=\sum_{i=1}^{r} \sum_{j \in \hat{J}_{i}} a_{i} p\left(\hat{\theta}_{i j}\right) \in C_{n}^{\operatorname{simp}, \inf }(K(N)),
$$

where $p: \widehat{K}(N) \rightarrow K(N)$ is the projection defined at the end of Section 3B, and $\tau_{i j}:=p\left(\hat{\theta}_{i j}\right)$ for all $i, j$.

Consider $Q \subset N$ as in the assumptions of Theorem 1.1. We define

$$
G:=\Pi\left(K\left(\partial_{0} Q\right)\right) .
$$

We have by assumption that $N=Q \cup R$ is an essential decomposition (as defined in the introduction), which means exactly that the assumptions of Lemma 3.5 are satisfied. Thus, according to Lemma 3.5, there exists a retraction

$$
r: C_{n}^{\operatorname{simp}, \inf }(K(N)) \otimes_{\mathbb{Z} G} \mathbb{Z} \rightarrow C_{n}^{\operatorname{simp}, \inf }(K(Q)) \otimes_{\mathbb{Z} G} \mathbb{Z}
$$

for $n \geq 2$, mapping $C_{n}^{\text {simp, inf }}(G K(\partial N)) \otimes_{\mathbb{Z} G} \mathbb{Z}$ to $C_{n}^{\text {simp, inf }}\left(G K\left(\partial_{1} Q\right)\right) \otimes_{\mathbb{Z} G} \mathbb{Z}$, such that, for each simplex $\tau_{i j} \in K(N)$, we either have $r\left(\tau_{i j} \otimes 1\right)=0$ or

$$
r\left(\tau_{i j} \otimes 1\right)=\kappa_{i j} \otimes 1
$$

for some simplex $\kappa_{i j} \in K(Q)$. (Recall that we've assumed that $n \geq 2$.) Thus

$$
r\left(\sum_{i=1}^{r} a_{i} \sum_{j \in \hat{J}_{i}} \tau_{i j} \otimes 1\right)=\sum_{i=1}^{r} a_{i} \sum_{j \in J_{i}} \kappa_{i j} \otimes 1
$$

with $J_{i} \subset \hat{J}_{i}$ for all $i$. (It may still be possible that $\left|J_{i}\right|=\infty$.) We remark that $\kappa_{i j}$ is only determined up to choosing one $\kappa_{i j}$ in its $G$-orbit.

Since $r$ is a chain map, we get a sufficient set of cancellations for $\sum_{i=1}^{r} a_{i} \sum_{j \in J_{i}} \kappa_{i j} \otimes 1$ by setting

$$
\mathscr{C}^{Q}:=\left\{\left(\partial_{k} \kappa_{i_{1} j_{1}} \otimes 1, \partial_{l} \kappa_{i_{2} j_{2}} \otimes 1\right):\left(\partial_{k} \tau_{i_{1} j_{1}}, \partial_{l} \tau_{i_{2} j_{2}}\right) \in \mathscr{C}^{N}\right\} .
$$

By assumption, $Q$ is aspherical. We can therefore apply Lemma 3.6 and get

$$
\partial\left(\sum_{i=1}^{r} a_{i} \sum_{j \in J_{i}} \kappa_{i j} \otimes 1\right) \in C_{*}^{\text {simp,inf }}\left(G K\left(\partial_{1} Q\right)\right) \otimes_{\mathbb{Z} G} \mathbb{Z}
$$

represents (the image of) $[\partial Q] \otimes 1$.

Lemma 3.4(a) gives that $G$ is amenable. Together with Lemma 3.7 this implies

$$
\|\partial Q\| \leq \sum_{i=1}^{r}\left|a_{i}\right|(n+1)\left|J_{i}\right| .
$$

In the remainder of the proof, we will use Lemma 5.16 to improve this inequality, getting rid of the unspecified (possibly infinite) numbers $\left|J_{i}\right|$. 
$Q, \partial Q, \partial_{0} Q, \partial_{1} Q$ satisfy Assumption I (page 135). Thus there exists a simplicial set

$$
K_{*}^{\mathrm{str}}(Q) \subset S_{*}(Q)
$$

satisfying conditions (i)-(viii) from Lemma 5.3, and a set

$$
D \subset K_{1}^{\mathrm{str}}(Q)
$$

of distinguished 1-simplices (Definition 5.8).

Recall that, for each $i$,

$$
\sum_{j \in \hat{J}_{i}} \theta_{i, j}
$$

was defined by choosing a triangulation of $\sigma_{i}^{-1}(N)$. The simplices $\theta_{i, j}$ thus have "old edges", i.e., subarcs of edges of $\sigma_{i}$, and "new edges", whose interior is contained in the interior of some subsimplex of $\sigma_{i}$ of dimension $\geq 2$.

Associated to $z=\sum_{i=1}^{r} a_{i} \sum_{j \in \hat{J}_{i}} \theta_{i j}$ and $\mathscr{C}^{N}$ (and an arbitrary minimal presentation of $\partial z$ ) are, by Definition 5.5, simplicial sets $\Upsilon^{N}, \partial \Upsilon^{N}$.

The only possibility that two old edges have a vertex in $\Upsilon^{N}$ in common is that this vertex is a vertex of $\sigma_{i}$.

So the labeling of edges of $\sum_{i=1}^{r} a_{i} \sum_{j \in \hat{J}_{i}} \theta_{i j}$ by labeling old edges not containing a vertex of any $\sigma_{i}$ with label 1 and all other edges with label 0 is an admissible labeling (Definition 5.10).

Associated to

$$
w=\sum_{i=1}^{r} a_{i} \sum_{j \in J_{i}} \kappa_{i j} \otimes 1
$$

and $\mathscr{C}^{Q}$ (and an arbitrary minimal presentation of $\partial w$ ) there are simplicial sets $\Upsilon, \partial \Upsilon$. By our definition of $\mathscr{C}^{Q}, \Upsilon$ is isomorphic to a simplicial subset of $\Upsilon^{N}$, namely to the subset generated by the set

$$
\left\{\tau \in \Upsilon^{N}: r(\tau \otimes 1) \neq 0\right\}
$$

together with all iterated faces and degenerations. In particular, the admissible 0-1 labeling of $\Upsilon^{N}$ induces an admissible 0-1 labeling of $\Upsilon$.

By Construction 5.1, there is a map of triples $q:\left(Q, \partial Q, \partial_{1} Q\right) \rightarrow\left(Q, \partial Q, \partial_{1} Q\right)$ which is (as a map of triples) homotopic to the identity, and such that $q\left(\partial_{0} Q \cap C\right)$ is path-connected for each path component $C$ of $\partial Q$.

We define

$$
A:=q\left(\partial_{0} Q\right), \quad H:=q_{*}(G)=q_{*}\left(\Pi\left(K\left(\partial_{0} Q\right)\right)\right) \subset \Pi(K(A)) .
$$

We observe that $H$ is a quotient of $G$, hence amenable, even though $\Pi(K(A))$ need not be amenable. 
Let $\widehat{\Upsilon}, \partial \widehat{\Upsilon}$ be defined by Observation 5.12. By Corollary 5.13, there is a chain map

$$
q \circ \operatorname{str}: C_{*}^{\text {simp,inf }}(\widehat{\Upsilon}) \otimes_{\mathbb{Z} G} \mathbb{Z} \rightarrow C_{*}^{\text {simp,inf }}\left(H K^{\text {str }}(Q)\right) \otimes_{\mathbb{Z} H} \mathbb{Z},
$$

mapping $C_{*}^{\text {simp,inf }}(\partial \widehat{\Upsilon}) \otimes_{\mathbb{Z} G} \mathbb{Z}$ to $C_{*}^{\text {simp, inf }}\left(H K^{\text {str }}\left(\partial_{1} Q\right)\right) \otimes_{\mathbb{Z} H} \mathbb{Z}$ such that

$$
\partial \sum_{i=1}^{r} a_{i} \sum_{j \in J_{i}} q\left(\operatorname{str}\left(\kappa_{i j}\right)\right) \otimes 1
$$

represents (the image of) $[\partial Q] \otimes 1$ and such that 1-labeled edges are mapped to distinguished 1-simplices. (We keep in mind that $\kappa_{i j}$ is only determined up to $G$-action; thus $q\left(\operatorname{str}\left(\kappa_{i j}\right)\right)$ is determined only up to choosing one simplex in its $H$-orbit.)

We then apply Lemma 5.16 to get the cycle

$$
\partial \sum_{i=1}^{r} a_{i} \sum_{j \in J_{i}} \operatorname{rmv}\left(q\left(\operatorname{str}\left(\kappa_{i j}\right)\right) \otimes 1\right) \in C_{*}^{\operatorname{simp}, \inf }\left(H K^{\mathrm{str}}\left(\partial_{1} Q\right)\right) \otimes_{\mathbb{Z} H} \mathbb{Z}
$$

representing (the image of) $[\partial Q] \otimes 1$. We want to show that this is actually a finite chain of $l^{1}$-norm at most

$$
(n+1) \sum_{i=1}^{r}\left|a_{i}\right|
$$

Claim. For each $i$,

$$
\partial \sum_{j \in J_{i}} \operatorname{rmv}\left(q\left(\operatorname{str}\left(\kappa_{i j}\right)\right) \otimes 1\right)
$$

is the formal sum of at most $n+1(n-1)$-simplices $L \otimes 1$ with coefficient 1 .

Proof. This is a consequence of the following subclaim and Lemma 4.13:

Assume that for some fixed $i \in I$, for the chosen triangulation

$$
\sigma_{i}^{-1}(N)=\bigcup_{j \in \hat{J}_{i}} \theta_{i j}
$$

and the associated canonical coloring, there exist $j_{1}, j_{2} \in \hat{J}_{i}$ and $k_{1}, k_{2} \in\{0, \ldots, n\}$ such that the faces

$$
T_{1}=\partial_{k_{1}} \theta_{i j_{1}} \in S_{n-1}(\partial N), \quad T_{2}=\partial_{k_{2}} \theta_{i j_{2}} \in S_{n-1}(\partial N)
$$

have a white-parallel arc (Definition 4.9). Then

$$
\operatorname{rmv}\left(q\left(\operatorname{str}\left(\kappa_{i j_{1}}\right)\right) \otimes 1\right)=0, \quad \operatorname{rmv}\left(q\left(\operatorname{str}\left(\kappa_{i j_{2}}\right)\right) \otimes 1\right)=0 .
$$

To prove the subclaim, note first that

$$
\partial_{k_{l}} \theta_{i j_{l}} \in S_{n-1}(\partial N)
$$

implies (by Lemma 3.5 and Construction 5.1)

$$
\partial_{k_{l}} q\left(\operatorname{str}\left(\kappa_{i j_{l}}\right)\right) \in H K_{*}^{\mathrm{str}}\left(\partial_{1} Q\right)
$$


for $l=1,2$. Now assume (for a contradiction) that

$$
\operatorname{rmv}\left(q\left(\operatorname{str}\left(\kappa_{i j_{1}}\right)\right) \otimes 1\right) \neq 0 .
$$

By the subclaim's hypothesis, there are white-parallel $\operatorname{arcs} e_{1}, e_{2}$ of $T_{1}$ and $T_{2}$, respectively. This means that there are $\operatorname{arcs} e_{1}, e_{2}$ in a 2-dimensional subsimplex $\tau^{2} \subset \Delta^{n}$ of the standard simplex, and subarcs $f_{1}, f_{2}$ of some edge of $\tau^{2}$, all satisfying

$$
\partial_{0} e_{1}=\partial_{1} f_{2}, \quad \partial_{0} f_{2}=\partial_{0} e_{2}, \quad \partial_{1} e_{2}=\partial_{0} f_{1}, \quad \partial_{1} f_{1}=\partial_{1} e_{1}
$$

and such that $e_{1}, f_{2}, e_{2}, f_{1}$ bound a square in the boundary of a white component. (See figure on page 154. We will use the same letter for an affine subset of $\Delta^{n}$ and for the singular simplex obtained by restricting $\sigma_{i}$ to this subset.) The square is of the form $U_{1}+U_{2}$, where $U_{1}, U_{2}$ are (n-2)-fold iterated faces of some $\theta_{i j}$ 's. Hence $\partial U_{1}=e_{1}+f_{2}+\partial_{2} U_{1}$ and $\partial U_{2}=-e_{2}-f_{1}-\partial_{2} U_{1}$, in other words,

$$
\partial\left(U_{1}+U_{2}\right)=e_{1}+f_{2}-e_{2}-f_{1} \quad \text { and } \quad \partial_{2} U_{1}=-\partial_{2} U_{2} .
$$

We emphasize that we assume $e_{1}$ and $e_{2}$ to be edges of $\theta_{i j_{1}}$ and $\theta_{i j_{2}}$, respectively, but $f_{1}, f_{2}$ need not be edges of $\theta_{i j_{1}}$ or $\theta_{i j_{2}}$.

Notational remark. For each iterated face $f=\partial_{k_{1}} \ldots \partial_{k_{l}} \theta_{i j}$ with $i \in I, j \in J_{i}$, we will denote by $f^{\prime}$ the $(n-l)$-simplex with

$$
f^{\prime} \otimes 1=\partial_{k_{1}} \ldots \partial_{k_{l}} \kappa_{i j} \otimes 1=r\left(\partial_{k_{1}} \ldots \partial_{k_{l}} \tau_{i j} \otimes 1\right)=r\left(\partial_{k_{1}} \ldots \partial_{k_{l}} p\left(\hat{\theta}_{i j}\right) \otimes 1\right) .
$$

(The last two equations are true because $r, p$ and the homotopy from $\sum_{i, j} a_{i} \theta_{i j}$ to $\sum_{i, j} \hat{\theta}_{i j}$ are chain maps.) In other words, if $f$ is an iterated face of some $\tau_{i j}$, then $f^{\prime}$ is, up to the ambiguity by the $H$-action, the corresponding iterated face of $\kappa_{i j}$.

By Lemma 3.5 we have $e_{1}^{\prime}, e_{2}^{\prime} \in G K\left(\partial_{1} Q\right)$. Thus we can (and will) choose $\kappa_{i j_{1}}, \kappa_{i j_{2}}$ in their $G$-orbits in such a way that $e_{1}^{\prime}, e_{2}^{\prime} \in K\left(\partial_{1} Q\right)$, which implies that $\operatorname{str}\left(e_{1}^{\prime}\right), \operatorname{str}\left(e_{2}^{\prime}\right) \in K^{\operatorname{str}}\left(\partial_{1} Q\right)$.

Since $r, p$ and the homotopy are chain maps, we have

$$
\partial_{2} U_{1}^{\prime} \otimes 1=-\partial_{2} U_{2}^{\prime} \otimes 1 .
$$

That is,

$$
\partial_{2} U_{1}^{\prime}=g \overline{\partial_{2} U_{2}^{\prime}}
$$

for some $g \in G$.

Since $U_{1}^{\prime}$ and $U_{2}^{\prime}$ belong to different $\kappa_{i j}$ 's, say $\kappa_{i j_{1}}$ and $\kappa_{i j_{2}}$, we can, upon replacing $\kappa_{i j_{2}}$ by $g \kappa_{i j_{2}}$, assume that

$$
\partial_{2} U_{1}^{\prime}=\overline{\partial_{2} U_{2}^{\prime}}
$$


that is, $U_{1}^{\prime}+U_{2}^{\prime}$ is a square. (Since $g$ maps $\partial e_{2}^{\prime}$ to $\partial e_{1}^{\prime}$, this second choice of $\kappa_{i j_{2}}$ in its $G$-orbit preserves the condition that $e_{2}^{\prime} \in K^{\mathrm{str}}\left(\partial_{1} Q\right)$.)

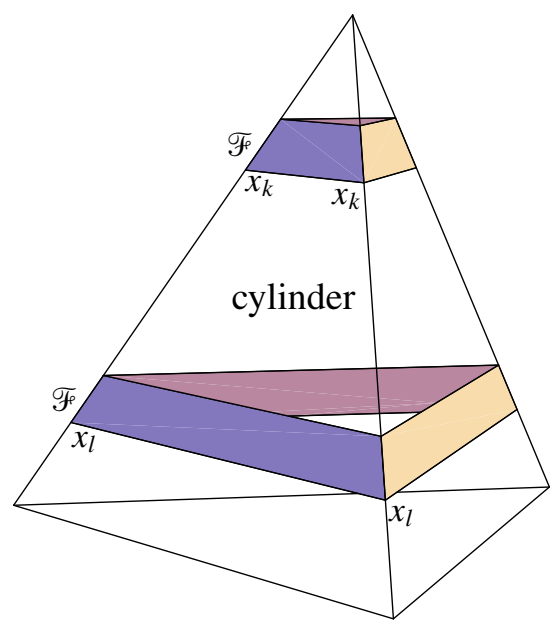

Let $F$ and $F^{\prime}$ be the path components of $\partial_{1} Q$ such that $e_{1}^{\prime} \subset F$ and $e_{2}^{\prime} \subset F^{\prime}$. Then $\partial_{1} \operatorname{str}\left(f_{1}^{\prime}\right), \partial_{0} \operatorname{str}\left(f_{2}^{\prime}\right) \in F$ and $\partial_{0} \operatorname{str}\left(f_{1}^{\prime}\right), \partial_{1} \operatorname{str}\left(f_{2}^{\prime}\right) \in F^{\prime}$.

We note that $f_{1}^{\prime}$ and $f_{2}^{\prime}$ are edges with label 1. By condition (i) of Corollary 5.13, this implies that $\operatorname{str}\left(f_{1}^{\prime}\right)$ and $\operatorname{str}\left(f_{2}^{\prime}\right)$ are distinguished 1-simplices.

By conditions (ix) and Condition (xiii) of Definition 5.8 we have

$$
\partial_{1} q\left(\operatorname{str}\left(f_{1}^{\prime}\right)\right)=x_{E_{0}^{F}}=\partial_{0} q\left(\operatorname{str}\left(f_{2}^{\prime}\right)\right), \partial_{0} q\left(\operatorname{str}\left(f_{1}^{\prime}\right)\right)=x_{E_{0}^{F^{\prime}}}=\partial_{1} q\left(\operatorname{str}\left(f_{2}^{\prime}\right)\right) .
$$

That is, $q\left(\operatorname{str}\left(e_{1}^{\prime}\right)\right)$ and $q\left(\operatorname{str}\left(e_{2}^{\prime}\right)\right)$ are loops in $\partial_{1} Q$, based respectively at $x_{E_{0}^{F}}$ and $x_{E_{0}^{F^{\prime}}}$.

Since the square $q\left(\operatorname{str}\left(U_{1}^{\prime}+U_{2}^{\prime}\right)\right)$ realizes a homotopy between $q\left(\operatorname{str}\left(f_{1}^{\prime}\right)\right)$ and $q\left(\operatorname{str}\left(f_{2}^{\prime}\right)\right)$, we have

$$
q\left(\operatorname{str}\left(f_{1}^{\prime}\right)\right)=\gamma_{1} q\left(\operatorname{str}\left(f_{2}^{\prime}\right)\right) \gamma_{2}
$$

with

$$
\gamma_{1}=q\left(\operatorname{str}\left(e_{1}^{\prime}\right)\right), \gamma_{2}=q\left(\operatorname{str}\left(e_{2}^{\prime}\right)\right) \in \Omega\left(\partial_{1} Q\right) \subset \Gamma=\Omega(\partial Q) .
$$

By condition (x) from Definition 5.8 this implies

$$
q\left(\operatorname{str}\left(f_{1}^{\prime}\right)\right)=q\left(\operatorname{str}\left(f_{2}^{\prime}\right)\right) .
$$

This means that $q\left(\operatorname{str}\left(U_{1}^{\prime}\right)\right)+q\left(\operatorname{str}\left(U_{2}^{\prime}\right)\right)$ is a cylinder with the boundary circles $q\left(\operatorname{str}\left(e_{1}^{\prime}\right)\right)$ and $q\left(\operatorname{str}\left(e_{2}^{\prime}\right)\right)$ in $\partial_{1} Q$.

(This is why we have performed the straightening construction in Section 5 in such a way that there should be only one distinguished 1-simplex in each coset.) 
The assumption $\operatorname{rmv}\left(q \circ \operatorname{str}\left(\kappa_{i j_{1}}\right) \otimes 1\right) \neq 0$ made at the top of page $161 \mathrm{implies}$ that the loops $q\left(\operatorname{str}\left(e_{1}^{\prime}\right)\right)$ and $q\left(\operatorname{str}\left(e_{2}^{\prime}\right)\right)$ are not 0-homotopic. Indeed, if one of them is 0 homotopic (and thus constant), so is the other, because they are homotopic through the cylinder. But $q\left(\operatorname{str}\left(e_{1}^{\prime}\right)\right)$ and $q\left(\operatorname{str}\left(e_{2}^{\prime}\right)\right)$ are edges of $q\left(\operatorname{str}\left(\kappa_{i j_{1}}\right)\right)$ and $q\left(\operatorname{str}\left(\kappa_{i j_{2}}\right)\right)$, respectively. In particular, $q\left(\operatorname{str}\left(\kappa_{i j_{1}}\right)\right)$ and $q\left(\operatorname{str}\left(\kappa_{i j_{2}}\right)\right)$ then have a constant loop as an edge. By Lemma 5.16 and Definition 5.2, this implies $\operatorname{rmv}\left(q \circ \operatorname{str}\left(\kappa_{i j_{1}}\right) \otimes 1\right)=0$.

Thus we can assume that $q\left(\operatorname{str}\left(e_{1}^{\prime}\right)\right)$ and $q\left(\operatorname{str}\left(e_{2}^{\prime}\right)\right)$ are not 0-homotopic, that is, the cylinder

$$
q\left(\operatorname{str}\left(U_{1}^{\prime}\right)\right)+q\left(\operatorname{str}\left(U_{2}^{\prime}\right)\right)
$$

is $\pi_{1}$-injective as a map of pairs. Since $\left(Q, \partial_{1} Q\right)$ is a pared acylindrical manifold, the cylinder must then be homotopic into $\partial Q$, as a map of pairs

$$
\left(\mathbb{S}^{1} \times[0,1], \mathbb{S}^{1} \times\{0,1\}\right) \rightarrow\left(Q, \partial_{1} Q\right) .
$$

Since $\partial_{1} Q$ is acylindrical, the cylinder must then either degenerate (that is, $\mathbb{S}^{1} \times$ $[0,1] \rightarrow \partial Q$ homotopes to a map that factors over the projection $\mathbb{S}^{1} \times[0,1] \rightarrow \mathbb{S}^{1}$; in particular, $\left.q\left(\operatorname{str}\left(e_{1}^{\prime}\right)\right)=q\left(\operatorname{str}\left(e_{2}^{\prime}\right)\right)\right)$ or be homotopic into $\partial_{0} Q$ (and hence into $q\left(\partial_{0} Q\right)$, since $\left.q \sim \mathrm{id}\right)$. In the second case the vertices $x_{E_{0}^{F}}, x_{E_{0}^{F^{\prime}}}$ must belong to $\partial_{0} Q$ and we get by condition (vii) from Lemma 5.3 that $q\left(\operatorname{str}\left(e_{1}^{\prime}\right)\right)$ and $q\left(\operatorname{str}\left(e_{2}^{\prime}\right)\right)$ lie in $K_{1}^{\operatorname{str}}\left(\partial_{0} Q\right)$. By Lemma 5.17 this implies that $q\left(\operatorname{str}\left(\kappa_{i j_{1}}\right)\right) \otimes 1$ and $q\left(\operatorname{str}\left(\kappa_{i j_{2}}\right)\right) \otimes 1$ vanish.

Thus we can assume that the cylinder degenerates. In particular, $q\left(\operatorname{str}\left(f_{1}^{\prime}\right)\right)$ and $q\left(\operatorname{str}\left(f_{2}^{\prime}\right)\right)$ lie in $K_{1}^{\mathrm{str}}\left(\partial_{1} Q\right)$.

Let $P_{1}, P_{2}$ be the affine planes whose intersections with $\Delta^{n}$ contain $T_{1}$ and $T_{2}$, respectively. Let $W$ be the white component whose boundary contains the whiteparallel $\operatorname{arcs}$ of $T_{1}, T_{2}$. We have seen that there are $\operatorname{arcs} f_{1}, f_{2}$ connecting $P_{1} \cap \Delta^{n}$ to $P_{2} \cap \Delta^{n}$ such that

$$
q\left(\operatorname{str}\left(f_{1}^{\prime}\right)\right), q\left(\operatorname{str}\left(f_{2}^{\prime}\right)\right) \in K_{1}^{\mathrm{str}}\left(\partial_{1} Q\right) .
$$

This implies that for each other arc $f$ connecting $P_{1} \cap \Delta^{n}$ to $P_{2} \cap \Delta^{n}$ the straightening $q\left(\operatorname{str}\left(f^{\prime}\right)\right)$ must be (homotopic into - and therefore, by condition (vii) from Lemma 5.3), contained in $-\partial_{1} Q$.

If $P_{1}$ and $P_{2}$ are of the same type (Definition 4.1), this shows that for all arcs $f \subset W$ we have

$$
q\left(\operatorname{str}\left(f^{\prime}\right)\right) \in K_{1}^{\mathrm{str}}\left(\partial_{1} Q\right)
$$

If $P_{1}$ and $P_{2}$ are not of the same type, then the existence of a parallel arc implies that at least one of them, say $P_{1}$, must be of type $\left\{0 a_{1} \ldots a_{k}\right\}$ with $k \notin\{0, n-1\}$. Then, for each plane $P_{3} \neq P_{1}$ with $P_{3} \cap \Delta^{n} \subset \partial W$, it follows from Corollary 4.8 that $P_{3} \cap \Delta^{n}$ has a white-parallel arc with $P_{1} \cap \Delta^{n}$. Thus, repeating the argument with $P_{1}$ and $P_{3}$ in place of $P_{1}$ and $P_{2}$, we prove that there are $\operatorname{arcs}$ in $\partial_{1} Q$ connecting $P_{1} \cap \Delta^{n}$ 
to $P_{3} \cap \Delta^{n}$, and consequently for each arc $f \subset W$ connecting $P_{1} \cap \Delta^{n}$ to $P_{3} \cap \Delta^{n}$, the straightening $\operatorname{str}\left(f^{\prime}\right)$ must be homotopic into, and thus contained in, $\partial_{1} Q$.

Consequently, also for arcs connecting $P_{2} \cap \Delta^{n}$ to $P_{3} \cap \Delta^{n}$, we conclude that $q\left(\operatorname{str}\left(f^{\prime}\right)\right)$ must be homotopic into, and therefore contained in, $\partial_{1} Q$. This finally shows that the 1-skeleta of $q\left(\operatorname{str}\left(\kappa_{i j_{1}}\right)\right)$ and $q\left(\operatorname{str}\left(\kappa_{i j_{2}}\right)\right)$ belong to $K_{1}^{\text {str }}\left(\partial_{1} Q\right)$. By the $\pi_{1}$-injectivity of $\partial_{1} Q \rightarrow Q$, the asphericity of $K\left(\partial_{1} Q\right)$, and condition (vii) from Lemma 5.3, this implies that the 2-skeleta of $q\left(\operatorname{str}\left(\kappa_{i j_{1}}\right)\right)$ and $q\left(\operatorname{str}\left(\kappa_{i j_{2}}\right)\right)$ belong to $K_{1}^{\mathrm{str}}\left(\partial_{1} Q\right)$. Inductively, if the $k$-skeleta of $q\left(\operatorname{str}\left(\kappa_{i j_{1}}\right)\right)$ and $q\left(\operatorname{str}\left(\kappa_{i j_{2}}\right)\right)$ belong to $K_{k}^{\text {str }}\left(\partial_{1} Q\right)$, then by the asphericity of $K(Q)$ and $K\left(\partial_{1} Q\right)$ together with condition (vii) from Lemma 5.3 we obtain that the $k+1$-skeleta of $q\left(\operatorname{str}\left(\kappa_{i j_{1}}\right)\right)$ and $q\left(\operatorname{str}\left(\kappa_{i j_{2}}\right)\right)$ belong to $K_{k+1}^{\mathrm{str}}\left(\partial_{1} Q\right)$. This provides the inductive step and thus our inductive proof shows that $q\left(\operatorname{str}\left(\kappa_{i j_{1}}\right)\right)$ and $q\left(\operatorname{str}\left(\kappa_{i j_{2}}\right)\right)$ belong to $K^{\mathrm{str}}\left(\partial_{1} Q\right)$.

By Definitions 5.2 and 5.14 and Lemma 5.16 this implies

$$
\operatorname{rmv}\left(q\left(\operatorname{str}\left(\kappa_{i j_{1}}\right)\right) \otimes 1\right)=0, \quad \operatorname{rmv}\left(q\left(\operatorname{str}\left(\kappa_{i j_{2}}\right)\right) \otimes 1\right)=0 .
$$

So we have shown the subclaim: if $T_{1}=\partial_{k_{1}} \theta_{i j_{1}}$ and $T_{2}=\partial_{k_{2}} \theta_{i j_{2}}$ have a whiteparallel arc, then $\operatorname{rmv}\left(q\left(\operatorname{str}\left(\kappa_{i j_{1}}\right)\right) \otimes 1\right)=0$ and $\operatorname{rmv}\left(q\left(\operatorname{str}\left(\kappa_{i j_{2}}\right)\right) \otimes 1\right)=0$. In particular, $q\left(\operatorname{str}\left(T_{1}^{\prime}\right)\right)$ and $q\left(\operatorname{str}\left(T_{2}^{\prime}\right)\right)$ do not occur (with nonzero coefficient) in

$$
\partial \sum_{j \in J_{i}} \operatorname{rmv}\left(q\left(\operatorname{str}\left(\kappa_{i j}\right)\right) \otimes 1\right) .
$$

By Lemma 4.13, for a canonical coloring associated to a set of affine planes $P_{1}, P_{2}, \ldots$, and a fixed triangulation of each $Q_{i}=P_{i} \cap \Delta^{n}$, we have at most $n+1$ $(n-1)$-simplices whose 1-skeleton does not contain a white-parallel arc. This show that the subclaim implies the claim.

The upshot is that we have presented $[\partial Q] \otimes 1$ as a finite chain of $l^{1}$-norm at most $(n+1) \sum_{i=1}^{r}\left|a_{i}\right|$. By Lemma 3.4(a) we know that $G=\Pi\left(K\left(\partial_{0} Q\right)\right)$ is amenable. Hence $H=q_{*}(G)$ is amenable. Thus Lemma 3.7, applied to $X=\partial Q$ and $K=H K^{\mathrm{str}}\left(\partial_{1} Q\right)$ with its $H$-action, implies $\|\partial Q\| \leq(n+1) \sum_{i=1}^{r}\left|a_{i}\right|$. This concludes the proof of Theorem 1.1.

Theorem 1.1 is not true without assuming the amenability of $\pi_{1} \partial_{0} Q$. Counterexamples can be found, for example, using [Jungreis 1997] or [Kuessner 2003, Theorem 6.3].

Remark. In [Agol 1999], Theorem 1.1 has been proven for incompressible surfaces in hyperbolic 3-manifolds. We compare the steps of the proof in [Agol 1999] with the arguments in our paper:

Agol's step 1 is the normalization procedure, which we restated in Lemma 2.4.

Step 2 consists in choosing compatible triangulations of the polytopes $\sigma_{i}^{-1}(N)$.

Step 3 boils down to the statement that, for each component $Q_{i}$ of $Q$, there exists a retraction $r: \hat{N} \rightarrow p^{-1}\left(Q_{i}\right)$, for the covering $p: \hat{N} \rightarrow N$ corresponding to 
$\pi_{1} Q_{i}$. Such a statement cannot be correct because it would (together with Agol's step 7) imply $\|N\| \geq\|Q\|$ whenever $Q$ is a $\pi_{1}$-injective submanifold of $N$. This inequality is true for submanifolds with amenable boundary, but not in general. In fact, one only has the more complicated retraction

$$
r: C_{*}\left(K(N), K\left(N^{\prime}\right)\right) \otimes_{\mathbb{Z} G} \mathbb{Z} \rightarrow C_{*}(K(Q), K(\partial Q)) \otimes_{\mathbb{Z} G} \mathbb{Z},
$$

with $G=\Pi\left(K\left(\partial_{0} Q\right)\right)$. This is why much of the latter arguments become notationally awkward, although conceptually not much is changing. Moreover, the action of $G$ is basically the reason why Theorem 1.1 is true only for amenable $G$.

Basically, the reason why the retraction $r: \hat{N} \rightarrow Q$ does not exist, is as follows. Let $R_{j}$ be the connected components of $\hat{N}-p^{-1}\left(Q_{i}\right)$. Then $R_{j}$ is homotopy equivalent to each connected component of $\partial R_{j}$. If $\partial R_{j}$ were connected for each $j$, this homotopy equivalence could be extended to a homotopy equivalence $r$ : $\hat{N} \rightarrow p^{-1}\left(Q_{i}\right)$. However, in most cases $\partial R_{j}$ will be disconnected, and then such an $r$ cannot exist.

We note that also the weaker construction of cutting off simplices does not work. A simplex may intersect $Q_{i}$ in many components and it is not clear which component to choose.

Step 4 from Agol's proof puts a hyperbolic metric with geodesic boundary on $Q$. His step 5 is the straightening procedure, corresponding to Sections $5 \mathrm{~B}-5 \mathrm{D}$ in this paper. We remark that the straightening procedure must be slightly more complicated than in [Agol 1999] because it is not possible, as suggested in that same paper, to homotope all edges between boundary components of $\partial Q$ into shortest geodesics. This is the reason why we can only straighten chains with an admissible 0-1 labeling of their edges (and why our straightening homomorphism in Section $5 \mathrm{C}$ is only defined on $C_{*}^{\text {simp }}(|\Upsilon|)$ and not on all of $C_{*}^{\text {sing }}(Q)$ ).

Agol's step 6 consists in removing degenerate simplices. This corresponds to Section $5 \mathrm{E}$ in this paper.

His step 7 proves that each triangle in $\sigma_{i}^{-1}(\partial N)$ contributes only once to the constructed fundamental cycle of $\partial Q$. Since, in our argument, we do not work with the covering $p: \hat{N} \rightarrow N$, we have no need for this justification.

His step 8 counts the remaining triangles per simplex (after removing degenerate simplices). It seems to have used the combinatorial arguments which we work out for arbitrary dimensions in Section 4.

We mention that the arguments of Section 4 are the only part of the proof which gets easier if one restricts to 3-manifolds rather than arbitrary dimensions. Moreover, the proof for laminations is the same as for hypersurfaces except for Lemma 2.4. Thus, upon these two points it seems that even in the case of incompressible surfaces in 3-manifolds the proof of Theorem 1.1 cannot be further simplified. 


\section{Specialization to 3-manifolds}

Guts of essential laminations. We start by recalling the guts-terminology. Let $M$ be a compact 3-manifold with (possibly empty) boundary consisting of incompressible tori, and $\mathscr{F}$ an essential lamination transverse or tangential to the boundary. $N=\overline{M-\mathscr{F}}$ is a, possibly noncompact, irreducible 3-manifold with incompressible, aspherical boundary $\partial N$. We set

$$
\partial_{0} N=\partial N \cap \partial M, \quad \partial_{1} N=\overline{\partial N-\partial_{0} N} .
$$

(Thus $\partial_{1} N$ is the union of boundary leaves of the lamination.) By the proof of [Gabai and Kazez 1998, Lemma 1.3], the noncompact ends of $N$ are essential $I$ bundles over noncompact subsurfaces of $\partial_{1} N$. After cutting off each of these ends along an essential, properly fibered annulus, one obtains a compact 3-manifold to which one can apply the JSJ-decomposition [Jaco and Shalen 1979; Johannson 1979]. Hence we have a decomposition of $N$ into the characteristic submanifold $\operatorname{Char}(N)$ (which consists of $I$-bundles and Seifert fibered solid tori, where the fibrations have to respect boundary patterns as defined in [Johannson 1979, p. 83]) and the guts of $N$, Guts $(N)$. The $I$-fibered ends of $N$ will be added to the characteristic submanifold, which thus may become noncompact, while $\operatorname{Guts}(N)$ is compact. (We mention that there are different notions of guts in the literature. Our notion is compatible with that of [Agol 1999; Agol et al. 2007], but differs from the definition in [Gabai and Kazez 1998] or [Calegari and Dunfield 2003] by taking the Seifert fibered solid tori into the characteristic submanifold and not into the guts. Thus, solid torus guts in the paper of Calegari-Dunfield is the same as empty guts in our setting.) If $\partial_{0} N \cap \partial Q \neq \varnothing$ consists of annuli $A_{1}, \ldots, A_{k}$, then, to be consistent with the setting of Theorem 1.1, we add components $A_{i} \times[0,1]$ to $\operatorname{Char}(N)$ (without changing the homeomorphism type of $N$ ), which implies $\partial_{0} N \cap \partial Q=\varnothing$.

For $Q=\operatorname{Guts}(N)$ we set

$$
\partial_{1} Q=\partial_{1} N \cap \partial Q=\partial N \cap \partial Q=Q \cap \partial N, \quad \partial_{0} Q=\overline{\partial Q-\partial_{1} Q} .
$$

For $R=\operatorname{Char}(N)$ we set

$$
\partial_{1} R=\partial N \cap \partial R, \quad \partial_{0} R=\overline{\partial R-\partial_{1} R} .
$$

Then $\partial_{0} N \cap \partial Q=\varnothing$ implies $\partial_{0} Q=Q \cap R$.

$\partial_{0} Q$ consists of essential tori and annuli; in particular $\pi_{1} \partial_{0} Q$ is amenable. The guts of $N$ has the following properties: the pair $\left(Q, \partial_{1} Q\right)$ is a pared acylindrical manifold (Definition 4.3), $Q, \partial_{1} Q, \partial_{1} R$ are aspherical, and the inclusions

$$
\partial_{0} Q \rightarrow Q, \quad \partial_{1} Q \rightarrow Q, \quad Q \rightarrow N, \quad \partial_{0} R \rightarrow R, \quad \partial_{1} R \rightarrow R, \quad R \rightarrow N
$$


are $\pi_{1}$-injective; see [Jaco and Shalen 1979; Johannson 1979]. It follows from Thurston's hyperbolization theorem for Haken manifolds that $Q$ admits a hyperbolic metric with geodesic boundary $\partial_{1} Q$ and cusps corresponding to $\partial_{0} Q$. (In particular, we have $\chi(\partial Q) \leq 0$; thus $\partial Q$ is aspherical, and $\partial_{1} Q$ is a hyperbolic surface, thus acylindrical.)

Theorem 7.1. Let $M$ be a compact 3-manifold with (possibly empty) boundary consisting of incompressible tori, and let $\mathscr{F}$ be an essential lamination of $M$. Then

$$
\|M, \partial M\|_{\mathscr{F}}^{\text {norm }} \geq-\chi(\operatorname{Guts}(\mathscr{F})) .
$$

More generally, if $P$ is a polyhedron with $f$ faces, then

$$
\|M, \partial M\|_{\mathscr{F}, P}^{\text {norm }} \geq-\frac{2}{f-2} \chi(\operatorname{Guts}(\mathscr{F})) .
$$

Proof. Let $N=\overline{M-\mathscr{F}}$. Since $\mathscr{F}$ is essential, $N$ is irreducible (hence aspherical, since $\partial N \neq \varnothing$ ) and has incompressible, aspherical boundary. Let $R=\operatorname{Char}(N)$ be the characteristic submanifold and $Q=\operatorname{Guts}(N)$ be the complement of the characteristic submanifold of $N$. The discussion before Theorem 7.1 shows that the decomposition $N=Q \cup R$ satisfies the assumptions of Theorem 1.1.

From the computation of the simplicial volume for surfaces [Gromov 1982, section 0.2] and $\chi(Q)=\frac{1}{2} \chi(\partial Q)$ (which is a consequence of Poincaré duality for the closed 3-manifold $\left.Q \cup_{\partial Q} Q\right)$, it follows that

$$
-\chi(\operatorname{Guts}(\mathscr{F}))=-\frac{1}{2} \chi(\partial \operatorname{Guts}(\mathscr{F}))=\frac{1}{4}\|\partial \operatorname{Guts}(\mathscr{F})\| .
$$

Thus, the first claim is obtained as application of Theorem 1.1 to $Q=\operatorname{Guts}(\mathscr{F})$.

The second claim - the generalization to arbitrary polyhedra - is obtained as in [Agol 1999]. Namely, one uses the same straightening as above, and asks again how many nondegenerate 2-simplices may, after straightening, occur in the intersection of $\partial Q$ with some polyhedron $P_{i}$. In [Agol 1999, p. 11], it is shown that this number is at most $2 f-4$, where $f$ is the number of faces of $P_{i}$. The same argument as above then shows that

$$
\sum_{i=1}^{r}\left|a_{i}\right| \geq \frac{1}{2 f-4}\|\partial \operatorname{Guts}(\mathscr{F})\|
$$

giving the wanted inequality.

The following corollary applies, for example, to all hyperbolic manifolds obtained by Dehn-filling the complement of the figure-eight knot in $\mathbb{S}^{3}$. (It was proved in [Hatcher 1992] that each hyperbolic manifold obtained by Dehn-filling the complement of the figure-eight knot in $\mathbb{S}^{3}$ carries essential laminations.) 
Corollary 7.1. If $M$ is a finite-volume hyperbolic manifold with $\operatorname{Vol}(M)<2 V_{3}=$ $2.02 \ldots$, then $M$ carries no essential lamination $\mathscr{F}$ with

$$
\|M, \partial M\|_{\mathscr{F}, P}^{\text {norm }}=\|M, \partial M\|_{P}
$$

for all polyhedra $P$, and nonempty guts. In particular, there is no tight essential lamination with nonempty guts.

Proof. The derivation of this corollary from Theorem 1.2 is exactly the same as in [Agol 1999] for the usual (nonlaminated) Gromov norm. Namely, by [Sleator et al. 1988] (or [Agol 1999], end of Section 6) there exists a sequence $P_{n}$ of straight polyhedra in $\mathbb{H}^{3}$ with

$$
\lim _{n \rightarrow \infty} \frac{\operatorname{Vol}\left(P_{n}\right)}{f_{n}-2}=V_{3},
$$

where $f_{n}$ denotes the number of faces of $P_{n}$. Assuming that $M$ carries a lamination $\mathscr{F}_{\mathcal{F}}$ with $\|M, \partial M\|_{\mathscr{F}, P_{n}}^{\text {norm }}=\|M, \partial M\|_{P_{n}}$ for all $n$, one gets

$$
-\chi(\operatorname{Guts}(\mathscr{F})) \leq \frac{f_{n}-2}{2}\|M, \partial M\|_{\mathscr{F}, P_{n}}=\frac{f_{n}-2}{2}\|M, \partial M\|_{P_{n}} \leq \frac{f_{n}-2}{2} \frac{\operatorname{Vol}(M)}{\operatorname{Vol}\left(P_{n}\right)},
$$

which tends to

$$
\frac{\operatorname{Vol}(M)}{2 V_{3}}<1 .
$$

On the other hand, if Guts(F्F) is not empty, it is a hyperbolic manifold with nonempty geodesic boundary; hence $\chi(\operatorname{Guts}(\mathscr{F})) \leq-1$, giving a contradiction.

Definition 7.2. The Weeks manifold is the closed 3-manifold obtained by applying $\left(-\frac{5}{1},-\frac{5}{2}\right)$-surgery at the Whitehead link [Rolfsen 1976, p. 68].

It is known that the Weeks manifold is hyperbolic and that its hyperbolic volume is approximately $0.94 \ldots$ It is actually the hyperbolic 3-manifold of smallest volume.

Corollary 7.3 [Calegari and Dunfield 2003, Conjecture 9.7]. The Weeks manifold admits no tight lamination $\mathscr{F}$.

Proof. According to [Calegari and Dunfield 2003], the Weeks manifold cannot carry a tight lamination with empty guts. Since tight laminations satisfy $\|M\|_{\mathscr{F}, P}^{\text {norm }}=$ $\|M\|$ for each polyhedron (see Lemma 2.4), and since the Weeks manifold has volume smaller than $2 V_{3}$, it follows from Corollary 7.1 that it cannot carry a tight lamination with nonempty guts neither.

The same argument shows that a hyperbolic 3-manifold $M$ of volume less than $2 V_{3}$ and such that there is no injective homomorphism $\pi_{1} M \rightarrow \mathrm{Homeo}^{+}\left(\mathbb{S}^{1}\right)$ cannot carry a tight lamination, because it was shown in [Calegari and Dunfield 2003] that the existence of a tight lamination with empty guts implies the existence of an 
injective homomorphism $\pi_{1} M \rightarrow$ Homeo $^{+}\left(\mathbb{S}^{1}\right)$. Some methods for excluding the existence of injective homomorphisms $\pi_{1} M \rightarrow \mathrm{Homeo}^{+}\left(\mathbb{S}^{1}\right)$ have been developed in that same paper (which yielded in particular the nonexistence of such homomorphisms for the Weeks manifold, used in the corollary above), but in general it is still hard to apply this criterion to other hyperbolic 3-manifolds of volume $<2 V_{3}$.

As indicated in [Calegari 2003], an approach to a generalization of some of the above arguments to essential, nontight laminations, yielding possibly a proof for nonexistence of essential laminations on the Weeks manifold, could consist in trying to define a straightening of cycles (as in the proof of Lemma 2.4) upon possibly changing the essential lamination.

As a consequence of a result of Tao $\mathrm{Li}$, one can at least exclude the existence of transversely orientable essential laminations on the Weeks manifold.

Corollary 7.4. The Weeks manifold admits no transversely orientable essential lamination $\mathscr{F}$.

Proof. According to [Li 2006, Theorem 1.1], if a closed, orientable, atoroidal 3manifold $M$ contains a transversely orientable essential lamination, then it contains a transversely orientable tight essential lamination. Hence Corollary 7.4 is a direct consequence of Corollary 7.3.

\section{Higher dimensions}

We want to finish this paper showing that Theorem 1.1 is interesting also in higher dimensions. While in dimension 3 the assumptions of Theorem 1.1 hold for any essential lamination, it is likely that this will not be the case for many laminations in higher dimensions. However, the most straightforward, but already interesting, application of the inequality is Corollary 8.1, which means that, for a given negatively curved manifold $M$, we can give an explicit bound on the topological complexity of geodesic hypersurfaces. Such a bound seems to be new except, of course, in the 3-dimensional case, where it goes back to [Agol 1999] and (with no explicit constants) to [Hass 1995].

Corollary 8.1. Let $M$ be a compact Riemannian n-manifold of negative sectional curvature and finite volume. Let $F \subset M$ be a geodesic $(n-1)$-dimensional hypersurface of finite volume. Then

$$
\|F\| \leq \frac{n+1}{2}\|M\|
$$

Proof. Consider $N=\overline{M-F}$. $(N, \partial N)$ is acylindrical. This is well-known and can be seen as follows: assume that $N$ contains an essential cylinder; then the double $D N=N \cup_{\partial_{1} N} N$ contains an essential 2-torus. But, since $N$ is a negatively curved manifold with geodesic boundary, we can glue the Riemannian metrics to 
get a complete negatively curved Riemannian metric on $D N$. In particular, $D N$ contains no essential 2-torus, giving a contradiction.

Moreover, the geodesic boundary $\partial N$ is $\pi_{1}$-injective and negatively curved, thus aspherical. Therefore we can choose $Q=N$, in which case the other assumptions of Theorem 1.1 are trivially satisfied. From Theorem 1.1 we conclude that

$$
\|M\|_{F}^{\text {norm }} \geq \frac{1}{n+1}\|\partial N\| .
$$

The boundary of $N$ consists of two copies of $F$, hence $\|\partial N\|=2\|F\|$. The leaf space of $\widetilde{F} \subset \tilde{M}$ is a Hausdorff tree; thus Lemma 2.4(b) implies $\|M\|_{F}^{\text {norm }}=\|M\|$. The claim follows.

This statement should be read as follows: for a given manifold $M$ (with given volume) one has an upper bound on the topological complexity of compact geodesic hypersurfaces.

For hyperbolic manifolds one can use the Chern-Gauss-Bonnet theorem and the proportionality principle to reformulate Corollary 8.1 as follows: If $M$ is a closed hyperbolic $n$-manifold and $F$ a closed $(n-1)$-dimensional geodesic hypersurface, then $\operatorname{Vol}(M) \geq C_{n} \chi(F)$ for a constant $C_{n}$ depending only on $n$.

\section{Acknowledgements}

It is probably obvious that this paper is strongly influenced by Agol's preprint [Agol 1999]. The argument that a generalization of Agol's inequality would imply Corollary 7.3 is from [Calegari and Dunfield 2003].

\section{References}

[Agol 1999] I. Agol, "Lower bounds on volumes of hyperbolic Haken 3-manifolds", preprint, University of California, Davis, CA, 1999. arXiv math.GT/9906182

[Agol et al. 2007] I. Agol, P. A. Storm, and W. P. Thurston, "Lower bounds on volumes of hyperbolic Haken 3-manifolds", J. Amer. Math. Soc. 20:4 (2007), 1053-1077. MR 2008i:53086 Zbl 1155.58016

[Benedetti and Petronio 1992] R. Benedetti and C. Petronio, Lectures on hyperbolic geometry, Springer, Berlin, 1992. MR 94e:57015 Zbl 0768.51018

[Brittenham 1995] M. Brittenham, "Essential laminations and Haken normal form", Pac. J. Math. 168:2 (1995), 217-234. MR 96c:57028 Zbl 0838.57011

[Calegari 2000] D. Calegari, "The Gromov norm and foliations", Geom. Funct. Anal. 10:6 (2000), 1423-1447. MR 2002c:57026a Zbl 0974.57015

[Calegari 2003] D. Calegari, "Problems in foliations and laminations of 3-manifolds", pp. 297-335 in Topology and geometry of manifolds (Athens, GA, 2001), edited by G. Matić and C. McCrory, Proc. Sympos. Pure Math. 71, AMS, Providence, RI, 2003. MR 2005b:57053 Zbl 1042.57501

[Calegari and Dunfield 2003] D. Calegari and N. M. Dunfield, "Laminations and groups of homeomorphisms of the circle”, Invent. Math. 152:1 (2003), 149-204. MR 2005a:57013 Zbl 1025.57018 
[Fenley 2007] S. R. Fenley, "Laminar free hyperbolic 3-manifolds", Comment. Math. Helv. 82:2 (2007), 247-321. MR 2008g:57020 Zbl 1136.57015

[Francaviglia 2004] S. Francaviglia, "Hyperbolic volume of representations of fundamental groups of cusped 3-manifolds", Int. Math. Res. Not. 2004:9 (2004), 425-459. MR 2004m:57032 Zbl 1088. 57015

[Gabai 1999] D. Gabai, "Essential laminations and Kneser normal form", J. Differ. Geom. 53:3 (1999), 517-574. MR 2001m:57025 Zbl 1028.57012

[Gabai and Kazez 1998] D. Gabai and W. H. Kazez, "Group negative curvature for 3-manifolds with genuine laminations", Geom. Topol. 2 (1998), 65-77. MR 99e:57023 Zbl 0905.57011

[Gabai and Oertel 1989] D. Gabai and U. Oertel, "Essential laminations in 3-manifolds", Ann. Math. (2) 130:1 (1989), 41-73. MR 90h:57012 Zbl 0685.57007

[Gromov 1982] M. Gromov, "Volume and bounded cohomology", Inst. Hautes Études Sci. Publ. Math. 56 (1982), 5-99. MR 84h:53053 Zbl 0516.53046

[Hass 1995] J. Hass, "Acylindrical surfaces in 3-manifolds", Mich. Math. J. 42:2 (1995), 357-365. MR 96c:57031 Zbl 0862.57011

[Hatcher 1992] A. Hatcher, "Some examples of essential laminations in 3-manifolds", Ann. Inst. Fourier (Grenoble) 42:1-2 (1992), 313-325. MR 93e:57026 Zbl 0759.57006

[Ivanov 1985] N. V. Ivanov, "Foundations of the theory of bounded cohomology", Zap. Nauchn. Semin. Leningr. Otd. Mat. Inst. Steklova 143 (1985), 69-109. In Russian; translated in J. Sov. Math. 37 (1987), 1090-1114. MR 87b:53070 Zbl 0573.55007

[Jaco and Shalen 1979] W. H. Jaco and P. B. Shalen, Seifert fibered spaces in 3-manifolds, Mem. Amer. Math. Soc. 21:220, Amer. Math. Soc., Providence, RI, 1979. MR 81c:57010 Zbl 0415.57005

[Johannson 1979] K. Johannson, Homotopy equivalences of 3-manifolds with boundaries, Lecture Notes in Mathematics 761, Springer, Berlin, 1979. MR 82c:57005 Zbl 0412.57007

[Jungreis 1997] D. Jungreis, "Chains that realize the Gromov invariant of hyperbolic manifolds", Ergod. Theory Dyn. Syst. 17:3 (1997), 643-648. MR 98c:57013 Zbl 0882.57009

[Kuessner 2003] T. Kuessner, "Efficient fundamental cycles of cusped hyperbolic manifolds", Pac. J. Math. 211:2 (2003), 283-313. MR 2004j:57034 Zbl 1066.57026

[Kuessner 2004] T. Kuessner, "Foliated norms on fundamental group and homology", Topol. Appl. 144:1-3 (2004), 229-254. MR 2005g:57056 Zbl 1054.57032

[Kuessner 2010] T. Kuessner, "Multicomplexes, bounded cohomology and additivity of simplicial volume", preprint, Universität Münster, 2010, available at http://www.math.uni-muenster.de/reine/ u/kuessner/preprints/bc.pdf.

[Li 2006] T. Li, "Compression branched surfaces and tight essential laminations", preprint, Oklahoma State University, Stillwater, OK, 2006, available at https://www2.bc.edu/ taoli/tight.pdf.

[May 1967] J. P. May, Simplicial objects in algebraic topology, Van Nostrand Math. Studies 11, Van Nostrand, Princeton, NJ, 1967. Reprinted University of Chicago Press, 1992. MR 36 \#5942 Zbl 0165.26004

[Roberts et al. 2003] R. Roberts, J. Shareshian, and M. Stein, "Infinitely many hyperbolic 3-manifolds which contain no Reebless foliation”, J. Amer. Math. Soc. 16:3 (2003), 639-679. MR 2004e: 57023 Zbl 1012.57022

[Rolfsen 1976] D. Rolfsen, Knots and links, Publish or Perish, Berkeley, CA, 1976. MR 58 \#24236 Zbl 0339.55004

[Sleator et al. 1988] D. D. Sleator, R. E. Tarjan, and W. P. Thurston, "Rotation distance, triangulations, and hyperbolic geometry”, J. Amer. Math. Soc. 1:3 (1988), 647-681. MR 90h:68026 Zbl 0653.51017 
[Thurston 1980] W. P. Thurston, "The geometry and topology of 3-manifolds", lecture notes, Princeton University, 1980, available at http://library.msri.org/books/gt3m.

Received June 15, 2008.

THILO KUESSNER

kuessner@math.uni-muenster.de

MATHEMATISCHES INSTITUT

UNIVERSITÄT MÜNSTER

EINSTEINSTRASSE 62

D-48149 MÜNSTER

GERMANY 


\title{
PACIFIC JOURNAL OF MATHEMATICS
}

\author{
http://www.pjmath.org \\ Founded in 1951 by
}

E. F. Beckenbach (1906-1982) and F. Wolf (1904-1989)

\section{EDITORS}

V. S. Varadarajan (Managing Editor)

Department of Mathematics

University of California

Los Angeles, CA 90095-1555

pacific@math.ucla.edu

Vyjayanthi Chari

Department of Mathematics

University of California

Riverside, CA 92521-0135

chari@math.ucr.edu

\section{Robert Finn}

Department of Mathematics Stanford University

Stanford, CA 94305-2125

finn@math.stanford.edu

Kefeng Liu

Department of Mathematics

University of California

Los Angeles, CA 90095-1555

liu@math.ucla.edu
Darren Long

Department of Mathematics

University of California

Santa Barbara, CA 93106-3080

long@math.ucsb.edu

Jiang-Hua Lu

Department of Mathematics

The University of Hong Kong

Pokfulam Rd., Hong Kong jhlu@maths.hku.hk

Alexander Merkurjev

Department of Mathematics University of California

Los Angeles, CA 90095-1555 merkurev@math.ucla.edu
Sorin Popa

Department of Mathematics

University of California

Los Angeles, CA 90095-1555

popa@math.ucla.edu

Jie Qing

Department of Mathematics

University of California

Santa Cruz, CA 95064

qing@ cats.ucsc.edu

Jonathan Rogawski

Department of Mathematics

University of California

Los Angeles, CA 90095-1555

jonr@math.ucla.edu

\section{PRODUCTION}

pacific@math.berkeley.edu

Silvio Levy, Scientific Editor Matthew Cargo, Senior Production Editor

ACADEMIA SINICA, TAIPEI

CALIFORNIA INST. OF TECHNOLOGY

INST. DE MATEMÁTICA PURA E APLICADA

KEIO UNIVERSITY

MATH. SCIENCES RESEARCH INSTITUTE

NEW MEXICO STATE UNIV.

OREGON STATE UNIV.

\section{SUPPORTING INSTITUTIONS}

STANFORD UNIVERSITY
UNIV. OF BRITISH COLUMBIA
UNIV. OF CALIFORNIA, BERKELEY
UNIV. OF CALIFORNIA, DAVIS
UNIV. OF CALIFORNIA, LOS ANGELES
UNIV. OF CALIFORNIA, RIVERSIDE
UNIV. OF CALIFORNIA, SAN DIEGO
UNIV. OF CALIF., SANTA BARBARA

UNIV. OF CALIF., SANTA CRUZ

UNIV. OF MONTANA

UNIV. OF OREGON

UNIV. OF SOUTHERN CALIFORNIA

UNIV. OF UTAH

UNIV. OF WASHINGTON

WASHINGTON STATE UNIVERSITY

These supporting institutions contribute to the cost of publication of this Journal, but they are not owners or publishers and have no responsibility for its contents or policies.

See inside back cover or www.pjmath.org for submission instructions.

The subscription price for 2011 is US \$420/year for the electronic version, and \$485/year for print and electronic.

Subscriptions, requests for back issues from the last three years and changes of subscribers address should be sent to Pacific Journal of Mathematics, P.O. Box 4163, Berkeley, CA 94704-0163, U.S.A. Prior back issues are obtainable from Periodicals Service Company, 11 Main Street, Germantown, NY 12526-5635. The Pacific Journal of Mathematics is indexed by Mathematical Reviews, Zentralblatt MATH, PASCAL CNRS Index, Referativnyi Zhurnal, Current Mathematical Publications and the Science Citation Index.

The Pacific Journal of Mathematics (ISSN 0030-8730) at the University of California, c/o Department of Mathematics, 969 Evans Hall, Berkeley, CA 94720-3840, is published monthly except July and August. Periodical rate postage paid at Berkeley, CA 94704, and additional mailing offices. POSTMASTER: send address changes to Pacific Journal of Mathematics, P.O. Box 4163, Berkeley, CA 94704-0163.

PJM peer review and production are managed by EditFLOW ${ }^{\mathrm{TM}}$ from Mathematical Sciences Publishers.

PUBLISHED BY PACIFIC JOURNAL OF MATHEMATICS

at the University of California, Berkeley 94720-3840

A NON-PROFIT CORPORATION

Typeset in IATEX

Copyright $(2011$ by Pacific Journal of Mathematics 


\section{PACIFIC JOURNAL OF MATHEMATICS}

Volume $251 \quad$ No. $1 \quad$ May 2011

An analogue of the Cartan decomposition for $p$-adic symmetric spaces of split $\quad 1$ $p$-adic reductive groups

PATRICK DELORME and VINCENT SÉCHERRE

Unital quadratic quasi-Jordan algebras

RAÚL FELIPE

The Dirichlet problem for constant mean curvature graphs in $\mathbb{M} \times \mathbb{R}$ over unbounded domains

Abigail FolHa and Sofia Melo

Osgood-Hartogs-type properties of power series and smooth functions

BUMA L. FRIDMAN and DAOWEI MA

Twisted Cappell-Miller holomorphic and analytic torsions

RUNG-TZUNG HUANG

Generalizations of Agol's inequality and nonexistence of tight laminations

THILO KUESSNER

Chern numbers and the indices of some elliptic differential operators

PING LI

Blocks of the category of cuspidal $\mathfrak{s p}_{2 n}$-modules

VOLODYMYR MAZORCHUK and CATHARINA STROPPEL

A constant mean curvature annulus tangent to two identical spheres is Delauney

SUNG-HO PARK

A note on the topology of the complements of fiber-type line arrangements in $\mathbb{C P} \mathbb{P}^{2}$

Sheng-Li Tan, Stephen S.-T. YaU and Fei Ye

Inequalities for the Navier and Dirichlet eigenvalues of elliptic operators

QIAOLING WANG and CHANGYU XIA

A Beurling-Hörmander theorem associated with the Riemann-Liouville operator

XUECHENG WANG 